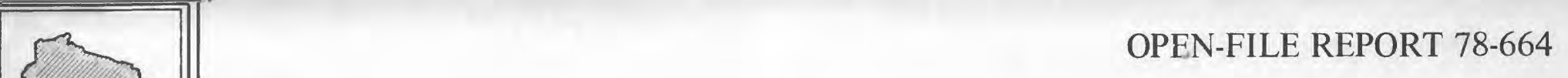

\title{
Low-Flow Characteristics of Small Streams in Proposed Public Law 566 Basins
}

PREPARED BY

UNITED STATES DEPARTMENT OF THE INTERIOR GEOLOGICAL SURVEY

IN COOPERATION WITH

WISCONSIN DEPARTMENT OF NATURAL RESOURCES 


\section{Low-Flow Characteristics of Small Streams in Proposed Public Law 566 Basins}

Stephen J. Field

U. S. GEOLOGICAL SURVEY

Open-File Report 78-664

Prepared in cooperation with the

Wisconsin Department of Natural Resources

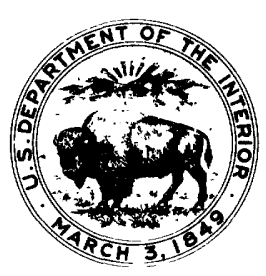

October 1978 


\section{UNITED STATES DEPARTMENT OF THE INTERIOR}

CECIL D. ANDRUS. SECRITARI

\section{GEOLOGICAL SURVEY}

H. William Menard, Director

For additional information write to:

U. S. Geological Survey

1815 University Avenue

Madison, Wisconsin 53706 


\section{CONTENTS}

Page

Abstract-_- 1

Introduction-_-__-____- 1

Low-flow characteristics-_-__- 2

Analytical techniques-_- 2

Gaging stations-_-_-_- 4

Low-flow partial-record stations-_-__-_-_ 4

Project stations-_-_- 4

Accuracy-_- 6

Gaging stations-_-__-_ 6

Low-flow partial-record stations-_-__-_-_-_-_-_-_-_-_-_ 6

Project stations-_-_- 6

References-_-_-__- 31

Index-_- 32

\section{ILLUSTRATIONS}

Figure 1. Map showing locations of Public Law 566 river basins where $Q_{7,2}$ and $Q_{7,10}$ have been estimated-

2. Graph showing method of estimating $Q_{7,2}$ and $Q_{7,10}$ from correlation of base-flow measurements at Sugar Creek near Troy to concurrent daily mean flow of Turtle

Creek near Clinton-_- 


\section{TABLES}

Table 1-15. Project site locations and low-flow characteristics for:

1. Lake Superior basin--_-n- 8

2. Duck-Pensaukee River basin-_-_-_-_-_-_-_ 9

3. Upper Fox River basin--_-_-_-_ 10

4. Wolf River basin-_-_-_-_-_-_-_-_-_ 13

5. Lower Fox River basin-___________-_ 15

6. Twin-Kewaunee River basin and Door County-- 16

7. Des Plaines and Pike River basin--.-.-.-.- 17

8. Trempealeau-Buffalo River-_-_-_-_-_-_--. 18

9. Black River basin-_-_-_-_-_-_-_-_-_-_- 18

10. Lower Wisconsin River basin-_-_-_-_-_-_-- 19

11. Grant-Platte River basin-_-_-_._-_-_-_--_ 22

12. Lower Rock River basin-_-_-_-_-_-_-_-_-_--- 23

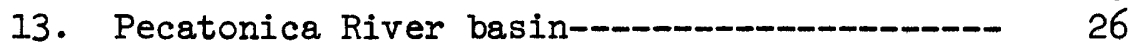

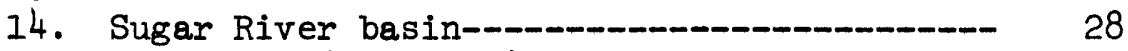

15. Fox River (Illinois) basin-_-_-_-_-_-- 29

16. Standard error of estimate for the 10-year low flow

$\left(\mathrm{SE}_{7}, 10\right)$ for major Wisconsin basins at low-flow partial-record stations--_-_-_-_-_-_-_-- 30 


\title{
Low-Flow Characteristics of Small Streams in Proposed Public Law 566 Basins
}

\author{
Stephen J. Field
}

\begin{abstract}
Low-flow characteristics of Wisconsin streams in basins considered for work under Public Law 566 are presented in this report. The low-flow characteristics presented are the annual minimum 7-day mean flow below which the flow will fall on the average of once in 2 years $\left(Q_{7}, 2\right)$ and the annual minimum 7-day mean flow below which the flow will fall on the average of once in 10 years $\left(Q_{7}, 10\right)$.

The low-flow characteristics are presented for 278 project sites in 32 basins considered for work under Public Law 566. They were determined by correlating base-flow measurements at the project site to the concurrent daily mean flow at continuous-record gaging stations in the area. The Q7,2 and $Q_{7,10}$ discharges determined by a frequency analysis at the continuousrecord gaging stations were used to estimate the $Q_{7,2}$ and $Q_{7,10}$ at the project sites.
\end{abstract}

The accuracy of the estimated low-flow characteristics is evaluated according to the type and amount of data available used. The statewide average of the accuracy determined by the standard error of estimate for the 10-year low flow ( $\left.\mathrm{SE}_{7}, 10\right)$, based on three base-flow measurements, is 45 percent but differs within the State.

\section{INTRODUCTION}

The purpose of this report is to describe the low-flow characteristics of Wisconsin streams in basins considered for watershed protection under Public Law 566. Public Law 566 is the Watershed Protection and Flood Prevention Act of 1954 enacted by the 83d Congress of the United States (Soil and Water Conservation Districts, 1973, p. 3). The study was done in cooperation with the Wisconsin Department of Natural Resources.

The Wisconsin Department of Natural Resources has the responsibility to assess the feasibility and the effects of land and water-management practices in the State. To accomplish this, the magnitude and frequency of 
the recurrence of low flows of streams must be known. Therefore, the annual minimum 7-day mean flow below which the flow will fall on the average of once in 10 years $(Q 7,10)$ and the annual minimum 7 -day mean flow below which the flow will fall on the average of once in 2 years $\left(Q_{7,2}\right)$ have been determined. In Wisconsin, the $Q_{7,10}$ is used as a guideline for water-quality standards and the $Q_{7,2}$ is used as a guideline for reservoirdischarge and water-use decisions. This report contains estimates of the Q7,2 and $Q 7,10$ at 278 sites in 32 basins being considered for work under Public Law 566. These basins are shown in figure 1.

For ease of reference the subbasins are listed alphabetically in the index. Also listed is the major basin to which it belongs and the page number where the data can be found.

For the convenience of readers who prefer to use metric units, the U.S. customary units in this report may be converted by using the following factors.

\section{MuItiply}

mile (mi)

foot ( $f t$ )

square mile $\left(\mathrm{mi}^{2}\right)$

cubic foot per second

$(\mathrm{ft} 3 / \mathrm{s})$

\section{By}

1.609

.3048

2.59

.028317
To obtain

kilometer $(\mathrm{km})$

meter $(\mathrm{m})$

square kilometer $\left(\mathrm{km}^{2}\right)$

cubic meter per second

$(\mathrm{m} 3 / \mathrm{s})$

\section{LOW-FLOW CHARACTERISTICS}

The estimated low-flow characteristics at the project sites appear in tables 1-15. These tables contain the low-flow characteristics at the project site locations in 15 of the 30 major basins (table 16) into which the Wisconsin Department of Natural Resources (DNR) has divided the State. The smaller project basins were listed under their respective major basin and are shown in tables 1-15. Information included in the tables for each site are stream name, station number, project site number, site location, drainage area, measured discharge, and accuracy level of the estimated lowflow characteristics. Sites that have no project site numbers were sites that were in the basins but not measured for this project. However, because the low-flow characteristics at these sites were determined for other projects, the data were included in this report.

\section{ANALYTICAL TECHNIQUES}

Low-flow characteristics in tables 1-15 have been estimated for three basic types of stations that involved different analytical methods. The three types of stations are: (1) continuous record of daily streamflow (continuous-record gaging stations); (2) 8 to 12 base-flow discharge measurements (low-flow partial-record stations); (3) 3 or more base-flow discharge measurements (stations established for the project). 

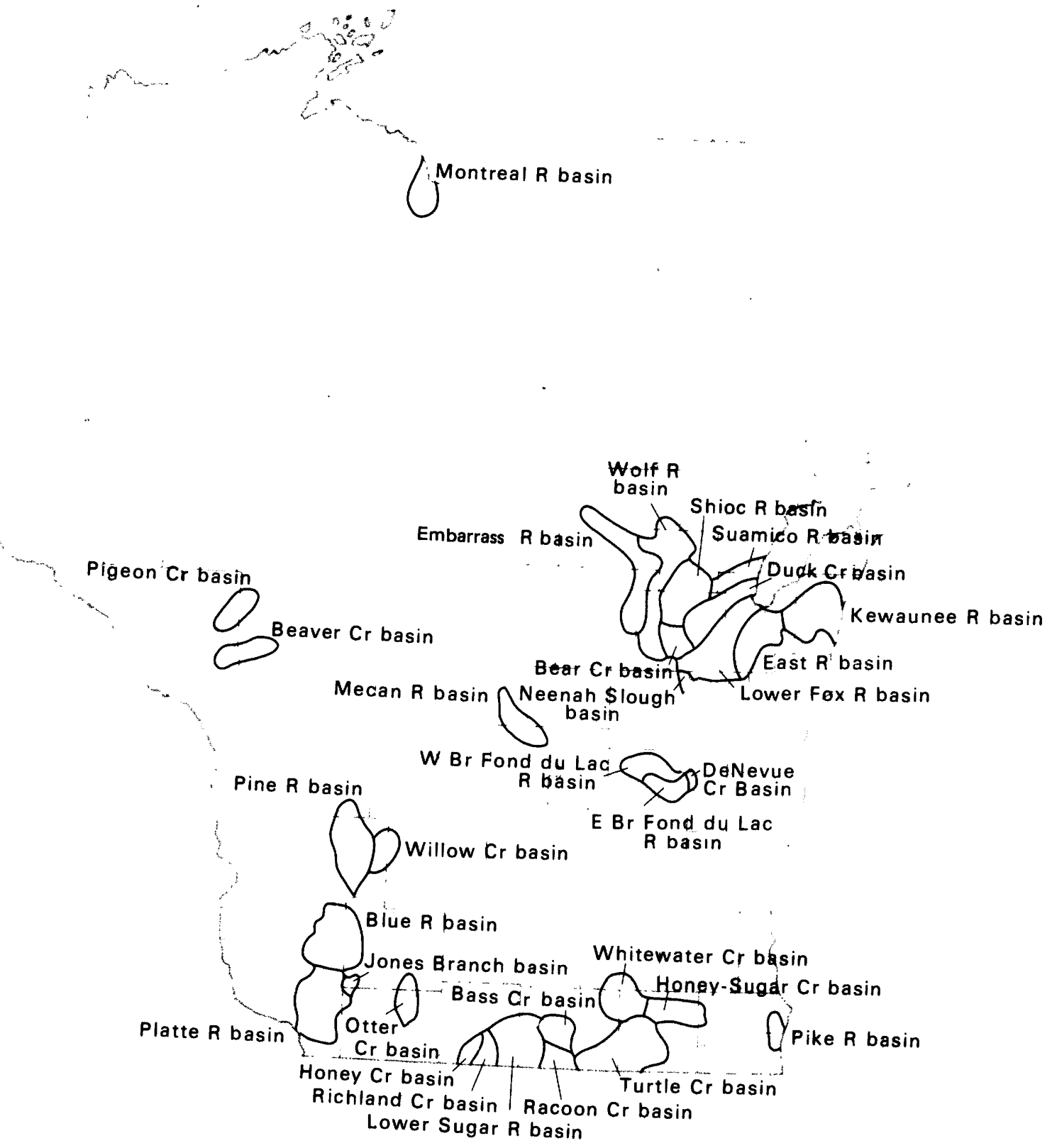

Figure 1. Locations of Public Law 566 river basins where

$Q_{7,2}$ and $Q_{7,10}$ have been estimated. 


\section{GAGING STATIONS}

The low-flow characteristics at gaging stations have been estimated by a frequency analysis of the recorded 7-day annual minimum flow by either a log-Pearson Type III probablility distribution or a graphical plot of the annual minimum 7-day mean flows (Riggs, 1972, p. 1-10). All the available data at gaging stations through the 1972 water year were used for this analysis except for basins in the lower Wisconsin, Grant-Platte, lower Rock, Pecatonica, Sugar, and Fox (Illinois) that used data through the 1975 water year.

\section{LOW-FLOW PARTIAL-RECORD STATIONS}

Low-flow characteristics at low-flow partial-record stations have been determined by a graphical correlation analysis. By correlating 8 to 12 base-flow discharge measurements at the low-flow partial-record station to the concurrent discharge at a nearby gaging station in the area a relation line was established (Gebert, 1971). The Q7,2 and Q7,10 at the continuousrecord gaging station then was transferred through the relation line to estimate $Q_{7,2}$ and $Q_{7,10}$ for the partial-record station.

\section{PROJECT STATIONS}

The estimated low-flow characteristics for project stations contained in this report are based on three or more base-flow measurements at each site. An exception to this is where one or two visits indicated no flow and the concurrent discharge at the gaging station used for correlation was greater than the $Q_{7,2}$. For these sites the $Q_{7,2}$ and $Q_{7,10}$ was assumed to be zero. The base-flow measurements at sites where three or more measurements were available were correlated with the concurrent daily mean flow at a continuous-record gaging station with similar hydrologic characteristics. The slope of the relation was compared to the established relation line at nearby low-flow partial-record stations for uniformity. If the factors that largely influence low flow are uniform in the area, the relation lines should have the same slope. The slope of relation line was adjusted to agree more closely to a regional slope, defined by the low-flow partialrecord stations, when the points defined by the three or more discharge measurements had a fairly large scatter. The Q7,2 and Q7,10 discharges, determined by a frequency analysis at the continuous-record gaging station then were transferred through the relation line to estimate the 07,2 and Q7,10 at the project sites. Figure 2 illustrates this method of correlation.

At some sites the relationships were not adequate to determine the low-flow characteristics and are designated by the letter " $a$ ". A few of these sites did not have enough range in discharge to define a relation line. Another resson for the poor definition of the relation line is that three measurements are not adequate in some basins. This is generally true when the basin of a gaging station has different hydrologic characteristics than the ungaged site. At these sites, additional measurements would be required to acquire the same degree of accuracy defined in basins with homogeneous hydrologic characteristics. 


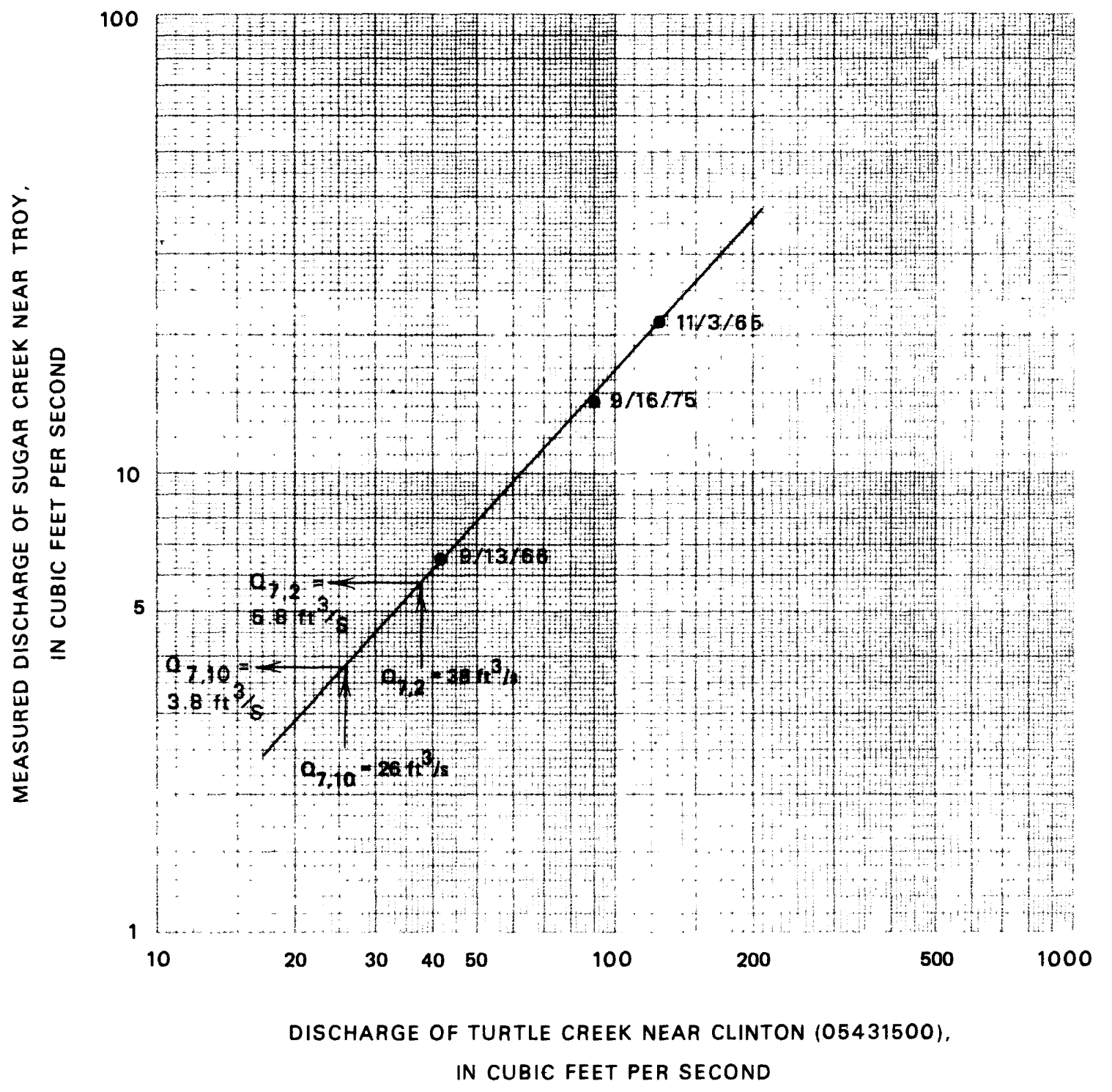

Figure 2. Method of estimating $Q_{7,2}$ and $Q_{7,10}$ from correlation of base-flow measurements at Sugar Creek near Troy to concurrent daily mean flow of Turtle Creek near Clinton. 


\section{ACCURACY}

The $Q_{7,2}$ and $Q_{7,10}$ low-flow characteristics in tables 1-15 are estimates of flow expected in the future. The estimates are based on data that were collected at each site and analyzed by several methods. Each estimate has an error associated with it, dependent on the amount and kind of data and on the analytical method. The two major sources of error in the estimates are the time-sampling error in collecting the data and the error in the analytical method.

Accuracy is determined by the standard error of estimate for the 10year low-flow discharge $\left(\mathrm{SE}_{7,10}\right)$. The estimated low-flow characteristics are within the $\mathrm{SE}_{7,10} 67$ percent of the time. An explanation of how the accuracy was determined for each method follows.

\section{GAGING STATIONS}

The accuracy for low-flow characteristics estimated from recorded discharge at continuous-record gaging stations was determined by Gebert and Holmstrom (1974, p. 7) using a frequency analysis described by Hardison (1969, p. D210-214). By analyzing all the gaging station records in Wisconsin with the assumption that 10 years of record were available at each site, they determined an $\mathrm{SE}_{7,10}$ of 18 percent. Gaging stations in the basins studied under Public Law 566 have the accuracy level of their SE7, 10 listed.

\section{LOW-FLOW PARTIAL-RECORD STATIONS}

The accuracy of low-flow characteristics at low-flow partial-record stations in Wisconsin was estimated by Gebert and Holmstrom (1974) using a method outlined by Hardison and Moss (1972, p. 42-45). Using this procedure they found an average $\mathrm{SE}_{7,10}$ of 29 percent for 265 stations. Low-flow partial-record stations in basins studied under Public Law 566 have the accuracy level of their $\mathrm{SE}_{7,10}$ listed in tables 1-15.

\section{PROJECT STATIONS}

Most of the project stations had less than six base-flow measurements and the accuracy of the low-flow characteristics of these stations cannot be appraised directly. However, Gebert and Holmstrom (1974, p. 7-8) used the data collected at low-flow partial-record stations to analyze the approximate accuracy associated with the low-flow characteristics based on only three random base-flow measurements. Their analyses resulted in an $\mathrm{SE}_{7,10}$ for the statewide average of 45 percent. Low-flow characteristics in tables 1-15 using this average have an accuracy level indicated by the letter " $b "$. If six or more discharge measurements were available at the project station, the $\mathrm{SE}_{7,10}$ was determined graphically and the accuracy listed.

The accuracy of the low-flow characteristics also differs for different areas of the State (table 16). These accuracies of Q7, 1.0 at low-flow 
partial-record stations probably are considerably better than those at the project sites because generally the low-flow characteristics can be defined better by more discharge measurements. This table can be used to evaluate the relative accuracy of low-flow estimates at project sites for different basins. For example, the $\mathrm{SE}_{7,10}$ at low-flow partial-record stations in the lower Wisconsin River basin is 11 percent, which is considerably lower than the average $S_{7,10}$ of 29 percent for all river basins in the State. Therefore, the $\mathrm{SE}_{7,10}$ for project sites in the lower Wisconsin River basin probably is less than the $\mathrm{SE}_{7,10}$ of 45 percent determined as the average for all river basins.

For those project stations where six or more previous base-flow measurements were available, a standard error of estimate was determined by measuring the standard error of the graphical regression of the log-relation plot (Riggs, 1968, p. 21-22). The accuracy of the low-flow characteristics at the project stations where six or more discharge measurements were available are listed in tables $1-15$. 
TABLE 1.--BASIN PROJECT SITE LOCATIONS AND LOH-FLOW CHADACTERISTICS

LAKE SUPERIOR BASIN

\begin{tabular}{|c|c|c|c|c|}
\hline \multirow[t]{2}{*}{$\begin{array}{l}\text { STATION } \\
\text { NO. }\end{array}$} & \multirow{2}{*}{\multicolumn{2}{|c|}{ STREAM NAME }} & \multirow[t]{2}{*}{$\begin{array}{l}\text { PROJECT } \\
\text { NO. }\end{array}$} & \multirow[b]{2}{*}{ MONTREAL RIVER BASIN } \\
\hline & & & & \\
\hline 04027852 & $\begin{array}{r}\text { MONTREAL } \\
\text { TRIBUT }\end{array}$ & $\begin{array}{l}\text { RIVER } \\
\text { TARY }\end{array}$ & 3 & $\begin{array}{l}\text { SW } 1 / 4 \text { SW } 1 / 4 \text { SEC. } 20, \text { T.44 N.S R. } 3 \text { E., } \\
\text { IRON COUNTY AT CULVERT ON U.S. HIGHWAY } \\
51.12 .6 \text { MI }(20.3 \mathrm{KM}) \text { SOUTH OF HURLEY. }\end{array}$ \\
\hline 04027876 & LAYMANS & CREEK & 6 & $\begin{array}{l}\text { SE } 1 / 4 \text { SW } 1 / 4 \text { SEC.1, T.44 N., R.2 E.P. } \\
\text { IRON COUNTY, AT CULVERT ON TOWN ROAD, } \\
9.3 \text { MI }(15.0 \mathrm{KM}) \text { SOUTH OF HURLEY. }\end{array}$ \\
\hline 04027990 & LAYMANS & CREEK & $i$ & $\begin{array}{l}\text { SW } 1 / 4 \text { NW } 1 / 4 \text { SEC.6, T.44 N., R.3 E. } \\
\text { IRON COUNTY, AROVE CONFLUENCF WITH } \\
\text { TRIBUTARY, } 9.0 \mathrm{MI}(14.5 \mathrm{KM}) \text { SOUTH OF } \\
\text { HURLEY. }\end{array}$ \\
\hline 04027890 & $\begin{array}{l}\text { LAYMANS } \\
\text { TRIBUT }\end{array}$ & $\begin{array}{l}\text { CREEK } \\
\text { TARY }\end{array}$ & 8 & $\begin{array}{l}\text { NW } 1 / 4 \text { SW } 1 / 4 \text { SEC.6. T.44 N.P R.3 E.Q } \\
\text { IPON CNUNTY, AT CULVERTS ON TOWN ROAD, } \\
9.1 \text { MI }(14.6 \text { KM) SOUTH OF HIRLEY. }\end{array}$ \\
\hline 04027805 & $\begin{array}{l}\text { LAYMANS } \\
\text { TRIRUT }\end{array}$ & $\begin{array}{l}\text { CREEK } \\
\text { TARY }\end{array}$ & 9 & $\begin{array}{l}\text { NF } 1 / 4 \text { NE } 1 / 4 \text { SEC.6, T. } 44 \text { N., R.3 E., } \\
\text { IRON CNUNTY, AT SCHOMBFRG PARK, B.S MI } \\
\text { (13.R KM) SOUTH OF HURLEY. }\end{array}$ \\
\hline 04027900 & LAYMANS & CREEK ${ }^{2}$ & lし & $\begin{array}{l}\text { NF } 1 / 4 \text { NF } 1 / 4 \text { SEC.6. T. } 44 \text { N.Q R. } 3 \text { E.P } \\
\text { IRON CNUNTY, AT RPIDGE ON U.S. HIGHWAY } \\
51 \text {. R.A MI }(14.2 \text { KM) SOUTH OF HURLEY. }\end{array}$ \\
\hline 040270155 & LAYMANS & CAEEK & 11 & $\begin{array}{l}\text { NW } 1 / 4 \text { NE } 1 / 4 \text { SEC.32. } 7.45 \text { N.. P.3 E., } \\
\text { IRON COUNTY, AT RRIDGE ON TOWN ROAN, } \\
\text { R.O MI (12.9 KM) SOITH OF HURLEY. }\end{array}$ \\
\hline $040270>0$ & MONTREAL & RIVFR & 12 & $\begin{array}{l}\text { CE } 1 / 4 \text { NE } 1 / 4 \text { SEC. } 28, T_{.45} N_{.} \text {R.3 E.. } \\
\text { IRON COUNTY, AT END OF TOWN HOAN. } \\
7.5 \text { MI }(12.1 \mathrm{KMI} \text { SOUTH OF HURLEY. }\end{array}$ \\
\hline 04027072 & $\begin{array}{l}\text { MONTREAL } \\
\text { TRIEHIT }\end{array}$ & $\begin{array}{l}\text { PIVFA } \\
\text { TARY }\end{array}$ & $1:$ & $\begin{array}{l}\text { NW } 1 / 4 \text { NW } 1 / 4 \text { 5EC.29, T.45 N., R.3 E.. } \\
\text { IRON COUNTY, AT CULVERT ON TOWN ROAD, } \\
5.8 \text { MI }(10.9 \text { KM) SOUTH OF HURLEY. }\end{array}$ \\
\hline 04027935 & $\begin{array}{l}\text { MONTREAL } \\
\text { TRIBUT }\end{array}$ & $\begin{array}{l}\text { RIVER } \\
\text { TARY }\end{array}$ & 14 & $\begin{array}{l}\text { NW } 1 / 4 \text { SW } 1 / 4 \text { SEC. } 20 . T .45 \text { N., R.3 E., } \\
\text { IRON COUNTY, AT CULVFRTS ON TOWN HOAD, } \\
\text { G.2 MI }(10.0 \text { KM) SNUTH OF HURLEY. }\end{array}$ \\
\hline $04 n 2704 n$ & $\begin{array}{l}\text { MONTREAL } \\
\text { TRIRUTT }\end{array}$ & $\begin{array}{l}\text { RIVER } \\
\text { TARY }\end{array}$ & Ib & 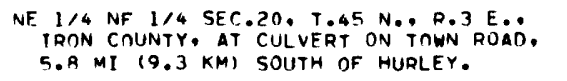 \\
\hline $040290 n 0$ & MONTREAL & $L$ RIVFR ${ }^{3}$ & 19 & $\begin{array}{l}\text { NW } 1 / 4 \text { SF } 1 / 4 \text { SEC.24, T.46 N.. R.2 E.. } \\
\text { IRON COUNTY, AT ARIOGE ON AURORA } \\
\text { STREET, BETWEEN HURLFY, WIS.. AND } \\
\text { IRONWOOD, MICH. }\end{array}$ \\
\hline
\end{tabular}

\begin{tabular}{|c|c|c|c|c|}
\hline $\begin{array}{l}\text { ORAIN- } \\
\text { AGE } \\
\text { AREA }\end{array}$ & DATE & $\begin{array}{l}\text { DIS- } \\
\text { CHARGE } \\
\left(F T^{3} / 5\right)\end{array}$ & $\begin{array}{l}\text { LOW-FLOW } \\
\text { CHARAC- } \\
\text { TERISTICS } \\
07.2 \text { Q } \\
\text { Q7.10 }\end{array}$ & $\begin{array}{l}\text { ACCU- } \\
\text { RACY } \\
\text { LEVFL } \\
\text { SE } 10 \\
\text { IPER- }\end{array}$ \\
\hline
\end{tabular}

\begin{tabular}{|c|c|c|c|c|c|}
\hline .59 & $\begin{array}{l}10-14-71 \\
06-14-73 \\
10-05-75\end{array}$ & $\begin{array}{l}.22 \\
.16 \\
.05\end{array}$ & 0.01 & $<0.01$ & $\mathrm{~b}$ \\
\hline .40 & $\begin{array}{l}10-13-71 \\
06-14-73 \\
10-05-75\end{array}$ & $\begin{array}{r}2.30 \\
3.48 \\
.30\end{array}$ & .11 & .04 & $b$ \\
\hline 5.12 & $\begin{array}{l}10-13-71 \\
n 5-14-73 \\
10-05-75\end{array}$ & $\begin{array}{r}4.65 \\
6.68 \\
.67\end{array}$ & $i^{\prime}$ & .08 & $\mathrm{~b}$ \\
\hline 9.00 & $\begin{array}{l}10-13-71 \\
06-14-73 \\
10-05-75\end{array}$ & $\begin{array}{l}5.52 \\
9.62 \\
1.56\end{array}$ & .36 & .77 & $v$ \\
\hline 2.17 & $\begin{array}{l}10-12-71 \\
n n-14-73 \\
10-05-75\end{array}$ & $\begin{array}{l}1.45 \\
1.65 \\
.62\end{array}$ & $.0 n$ &.$\cap$ & b \\
\hline 17.5 & $n 8-18-75$ & 2.14 &.\ulcorner & $\therefore$ & $Y^{\prime}$ \\
\hline 19.4 & $\begin{array}{l}10-13-71 \\
n x-14-73 \\
10-05-75\end{array}$ & $\begin{array}{c}14.1 \\
15.3 \\
3.16\end{array}$ & Firi & $\cdots "$ & b \\
\hline 30.1 & $\begin{array}{l}10-12-71 \\
06-14-73 \\
10-04-75\end{array}$ & $\begin{array}{l}28.0 \\
37.6 \\
14.0\end{array}$ & 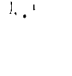 & $\cdot$ & $t$ \\
\hline . $6 B$ & $\begin{array}{l}10-13-71 \\
05-14-73 \\
10-06-75\end{array}$ & $\begin{array}{l}.37 \\
.23 \\
.05\end{array}$ & .01 & $-.0 !$ & b \\
\hline 1.84 & $\begin{array}{l}10-12-71 \\
n 6-14-73 \\
10-06-75\end{array}$ & $\begin{array}{l}.61 \\
.53 \\
.07\end{array}$ & .05 & .01 & $t$ \\
\hline 3.03 & $\begin{array}{l}10-12-71 \\
06-14-73 \\
10-104-75\end{array}$ & $\begin{array}{l}.93 \\
.49 \\
.05\end{array}$ & .7 & $.0^{-}$ & br \\
\hline 63.8 & $09-26-25$ & .10 & 4.7 & 2,1 & $=0$ \\
\hline
\end{tabular}

1. $N-F^{2}, H$ TARTIAL-RECORD STATION.

3. LI MU:A MEAOUEED

MINIMUM RECURDED. 
TABLE 2.- BASIN PROJECT SITE LOCATIONS ANO LOW-FLOW CHARACTERISTICS

DUCK-PENSAUKEE RIVER BASIN

\begin{tabular}{|c|c|c|c|c|c|c|c|c|c|}
\hline \multirow[t]{2}{*}{$\begin{array}{l}\text { STATION } \\
\text { NO. }\end{array}$} & \multirow[t]{2}{*}{ STREAM NAME } & \multirow[t]{2}{*}{$\begin{array}{l}\text { PROJECT } \\
\text { NO. }\end{array}$} & \multirow[b]{2}{*}{ SUAMICO RIYVER BASIN } & \multirow[t]{2}{*}{$\begin{array}{l}\text { DRAIN- } \\
\text { AGE } \\
\text { AREA } \\
\text { (MI2) }\end{array}$} & \multirow[t]{2}{*}{ DATE } & \multirow[t]{2}{*}{$\begin{array}{l}\text { DIS- } \\
\text { CHARGE } \\
\text { (FT } 3 / S)\end{array}$} & \multirow{2}{*}{\multicolumn{2}{|c|}{$\begin{array}{l}\text { CHARAC- } \\
\text { TERISTICS } \\
07 \% 2 \quad 07,10 \\
(F T=3)(F T 3 / 5)\end{array}$}} & \multirow[t]{2}{*}{$\begin{array}{l}\text { LEVEL } \\
\text { SE LO } \\
\text { (PER- } \\
\text { CENT) }\end{array}$} \\
\hline & & & & & & & & & \\
\hline 04071903 & $\begin{array}{l}\text { LITTLE SUAMICO } \\
\text { RIVER }\end{array}$ & 4 & $\begin{array}{l}\text { NE } 1 / 4 \text { NE } 1 / 4 \text { SEC.24, T.26 N., R.19 E., } \\
\text { OCONTO COUNTY, AT BRIDGE ON TOWN ROAO, } \\
3.0 \mathrm{MI}(4.8 \mathrm{KM}) \text { WEST OF SOBIESKI. }\end{array}$ & $34 \cdot 8$ & $\begin{array}{l}08-13-69 \\
08-27-69 \\
07-08-76\end{array}$ & $\begin{array}{l}1.67 \\
1.27 \\
1.28\end{array}$ & 0.78 & 0.45 & $\mathrm{~b}$ \\
\hline 04071915 & $\begin{array}{l}\text { LITTLE SUAMICO } \\
\text { RIVER }\end{array}$ & & $\begin{array}{l}\text { SE 1/4 SE 1/4 SEC.24, T.26 N., R.20 E.. } \\
\text { OCONTO COUNTY, AT BRIDGE ON COUNTY } \\
\text { TRUNK HIGHWAY J, O.3 MI }(0.5 \mathrm{KM}) \text { SOUTH } \\
\text { OF LITTLE SUAMICO. }\end{array}$ & $54 \cdot 8$ & $\begin{array}{l}08-13-69 \\
08-28-69 \\
10-17-72 \\
07-08-76 \\
07-14-76\end{array}$ & $\begin{array}{l}1.46 \\
.66 \\
9.73 \\
.64 \\
.29\end{array}$ & .30 & .07 & $\mathrm{~b}$ \\
\hline 04071965 & $\begin{array}{l}\text { SUAMICO RIVER } \\
\text { TRIBUTARY }\end{array}$ & 2 & $\begin{array}{l}\text { SE } 1 / 4 \text { NW } 1 / 4 \text { SEC.22, T.25 N., R.19 E., } \\
\text { AROWN COUNTY, ON TOWN ROAD, } \\
\text { O.7 MI (1.1 KM) NORTHEAST OF } \\
\text { KUNESH. }\end{array}$ & $12 \cdot 0$ & $\begin{array}{l}08-12-69 \\
08-28-69\end{array}$ & $\begin{array}{l}0.0 \\
0.0\end{array}$ & 0.0 & 0.0 & $\mathrm{~b}$ \\
\hline 04072000 & SUAMICO RIVER ${ }^{3}$ & 5 & $\begin{array}{l}\text { NW } 1 / 4 \text { NW } 1 / 4 \text { SEC.22, T.25 N.. R.20 E.. } \\
\text { BROWN COUNTY, AT BRIDGE ON COUNTY TRUNK } \\
\text { HIGHWAY B, O.5 MI }(0.8 \mathrm{KM}) \text { WEST OF } \\
\text { SUAMICO, } 7.5 \text { MI }(12.1 \mathrm{KM}) \text { NORTH OF } \\
\text { GREEN AAY, } 3.0 \mathrm{MI}(4.8 \mathrm{KM}) \text { UPSTREAM } \\
\text { FROM MOUTH. }\end{array}$ & $57 \cdot 0$ & $08-26-69$ & ${ }^{2} 2.53$ & 1.3 & .74 & 79 \\
\hline & & & DUCK CREEK BASIN & & & & & & \\
\hline $0407 ? 015$ & DUCK CREEK & 1 & $\begin{array}{l}\text { SE 1/4 NE } 1 / 4 \text { SEC.8, T.22 N., R.18 E., } \\
\text { OUTAGAMIE COUNTY, AT BRIDGE ON COUNTY } \\
\text { TRUNK HIGHWAY C, } 8.0 \text { MI } 112.9 \text { KMI } \\
\text { SOUTHEAST OF BLACK CREEK. }\end{array}$ & 32.0 & $08-12-69$ & 0.0 & 0.0 & 0.0 & $\mathrm{~b}$ \\
\hline $0407>021$ & $\begin{array}{l}\text { DUCK CREEK } \\
\text { TRIBUTARY }\end{array}$ & 2 & $\begin{array}{l}\text { SE } 1 / 4 \text { NE } 1 / 4 \text { 5EC. } 20, \text { T.?2 N., R. } 18 \text { E. } \\
\text { OUTAGAMIE COUNTY, AT BRIDGE ON COUNTY } \\
\text { TRUMK HIGHWAY C, } 4.2 \text { MI }(6.8 \mathrm{KM}) \text { EAST } \\
\text { OF MACKVILLE. }\end{array}$ & $7 . A 1$ & $08-12-69$ & 0.0 & 0.0 & 0.0 & $\mathrm{~b}$ \\
\hline 04072024 & NUCK CREFK & & $\begin{array}{l}\text { SE } 1 / 4 \text { SF } 1 / 4 \text { SEC.16, T.22 N., R.18 E.. } \\
\text { OUTAGAMIE COUNTY, AT BRIOGE ON } \\
\text { VINE RO. } 1.4 \mathrm{MI}(2.3 \mathrm{KM}) \text { SOUTHWEST } \\
\text { OF FREEDOM. }\end{array}$ & $45 \cdot 3$ & $\begin{array}{l}10-17-72 \\
07-14-76\end{array}$ & $\begin{array}{l}4.53 \\
0.0\end{array}$ & 0.0 & 0.0 & $\mathrm{~b}$ \\
\hline $0407>031$ & DUCK CPEEK & 3 & $\begin{array}{l}\text { NW } 1 / 4 \text { NF } 1 / 4 \text { SEC.11. T. } P 2 \text { N.. R.18 E. } \\
\text { NUTAGAMIE COUNTY, AT BRIDGE ON COUNTRY } \\
\text { ROAD. } 1.1 \text { MI }(1.8 \text { KM) NORTH DF FREEDOM. }\end{array}$ & 47.3 & $08-12-69$ & 0.0 & 0.0 & 0.0 & $\mathrm{~b}$ \\
\hline $0407>041$ & $\begin{array}{l}\text { DUCK CPEEK } \\
\text { TRIRUTARY }\end{array}$ & 4 & $\begin{array}{l}\text { SE 1/4 SF } 1 / 4 \text { SEC.19, T.P3 N., R.19 E.. } \\
\text { OUTAGAMIE COUNTY, AT BRIDGE ON COUNTY } \\
\text { TRUNK HIGHWAY EE, } 4.6 \text { MI } 17.4 \text { KM) } \\
\text { SOUTHWEST OF ONEIDA. }\end{array}$ & $16 \cdot A$ & $08-12-69$ & 0.0 & 0.0 & 0.0 & $\mathrm{~b}$ \\
\hline 04072050 & DUCK CPEEK ${ }^{1}$ & $F_{1}$ & $\begin{array}{l}\text { SE } 1 / 4 \text { SEC.17, T.23 N., R.19 F. } \\
\text { RROWN COUNTY, AT BRIDGE ON COUNTRY } \\
\text { POAD, } 2.9 \text { MI }(4.7 \mathrm{KM}) \text { SOUTHWEST } \\
\text { OF ONEIOA. }\end{array}$ & 9?.? & $07-27-77$ & ${ }^{2} .00$ & .06 & .01 & 139 \\
\hline 04072150 & DUCK CREEK & $\epsilon_{1}$ & 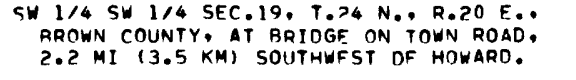 & $105 . n$ & $\begin{array}{l}0 B-11-69 \\
08-27-69 \\
07-07-76\end{array}$ & $\begin{array}{l}.94 \\
0.0 \\
<.01\end{array}$ & 0.0 & 0.0 & b \\
\hline $0407>195$ & TRNUT CAFFK & 7 & $\begin{array}{l}\text { NE } 1 / 4 \text { SE } 1 / 4 \text { SEC.24, T.24 N., R.19 E. } \\
\text { RROWN COUNTY, AT CULVERT ON TOWN ROAO, } \\
2.2 \text { MI }(3.5 \mathrm{KM}) \text { SOUTHWEST OF HOWARD. }\end{array}$ & 19.4 & $\begin{array}{l}08-11-69 \\
08-27-59 \\
n 7-08-76\end{array}$ & $\begin{array}{l}.43 \\
.24 \\
.70\end{array}$ & a & a & \\
\hline
\end{tabular}

${ }^{1}$ LWN-FLUW PARTIAL-RECORD STATION.

MIAL MITM VEASURED.

S J JII : BTATION. 


\begin{tabular}{|c|c|c|c|c|c|c|c|c|c|}
\hline \multicolumn{10}{|c|}{$\begin{array}{ll}\text { UPPER FOX RIVER BASIN } & \text { RP }\end{array}$} \\
\hline \multirow[t]{2}{*}{$\begin{array}{l}\text { STATION } \\
\text { NO. }\end{array}$} & \multirow[t]{2}{*}{ STREAM NAME } & \multirow[t]{2}{*}{$\begin{array}{l}\text { PROJECT } \\
\text { NO. }\end{array}$} & \multirow[b]{2}{*}{ MECAN RIVER BASIN } & \multirow[t]{2}{*}{$\begin{array}{l}\text { ORAIN= } \\
\text { AGE } \\
\text { AREA } \\
\text { (MI } 2 \text { ) }\end{array}$} & \multirow[t]{2}{*}{ DATE } & \multirow[t]{2}{*}{$\begin{array}{l}\text { DIS- } \\
\text { CHARGE } \\
(F T 3 / S)\end{array}$} & \multirow{2}{*}{\multicolumn{2}{|c|}{$\begin{array}{l}\text { LOW-FLOW } \\
\text { CHARAC- } \\
\text { TERISTICS } \\
Q 7,2 \text { QT, } 10 \\
\text { (FT3/S) }(F T 3 / 5)\end{array}$}} & \multirow[t]{2}{*}{$\begin{array}{l}\text { ACCU- } \\
\text { RACY } \\
\text { LEVEL } \\
\text { SE } 10 \\
\text { (PER- } \\
\text { CENTI }\end{array}$} \\
\hline & & & & & & & & & \\
\hline 04073190 & MECAN RIVER & 3 & $\begin{array}{l}\text { NE } 1 / 4 \text { NW } 1 / 4 \text { SEC. } 21, \text { T.1B N., R.9 E., } \\
\text { WAUSHARA COUNTY, AT CULVERT ON COUNTY } \\
\text { TRUNK HIGHWAY B, AT RICHFORD. }\end{array}$ & -- & $\begin{array}{l}08-25-70 \\
08-31-71 \\
09-21-71 \\
07-14-76\end{array}$ & $\begin{array}{l}14 \cdot 2 \\
20 \cdot 3 \\
18 \cdot 3 \\
20.6\end{array}$ & 13 & 7.6 & $\mathrm{~b}$ \\
\hline 04073245 & CHAFFEE CREEK & 11 & $\begin{array}{l}\text { NW } 1 / 4 \text { NW } 1 / 4 \text { SEC.5, T.17 N.9 R.9 E. } \\
\text { MARQUETTE COUNTY, AT BRIDGE ON COUNTY } \\
\text { TRUNK HIGHWAY JJ, 3.3 MI }(5.3 \mathrm{KMI} \\
\text { SOUTMUEST OF RICHFORD. }\end{array}$ & - & $\begin{array}{l}08-25-70 \\
09-01-71 \\
07-14-76\end{array}$ & $\begin{array}{l}14.3 \\
15.4 \\
16.0\end{array}$ & 13 & 9.4 & $\mathrm{~b}$ \\
\hline 04073300 & CHAFFEE CREEK ${ }^{1}$ & 17 & $\begin{array}{l}\text { NE } 1 / 4 \text { NE } 1 / 4 \text { SEC.18, T.17 N., R.10 E.., } \\
\text { MARQUETTE COUNTY, AT BRIDGE ON TOWN } \\
\text { ROAB, } 6.5 \text { MI }(10.5 \text { KM) WEST OF } \\
\text { NESWKORO. }\end{array}$ & $45 \cdot 4$ & $n 8-25-70$ & 225.9 & 26 & 18 & 11 \\
\hline 04073315 & $\begin{array}{l}\text { MECAN RIVER } \\
\text { TRIBUTARY }\end{array}$ & 19 & $\begin{array}{l}\text { NW } 1 / 4 \text { NW } 1 / 4 \text { SEC. } 27, T \text { T } 17 \text { N.. R.10 E., } \\
\text { MAREUETTE COUNTY, AT BRIDGE ON DUCK } \\
\text { CREEK AVENUE, } 5.2 \text { MI }(8.4 \mathrm{KM}) \text { SOUTHWEST } \\
\text { OF NESHKORO. }\end{array}$ & -- & $\begin{array}{l}08-25-70 \\
09-01-71\end{array}$ & $\begin{array}{l}0.00 \\
0.00\end{array}$ & 0.0 & 0.0 & $\mathrm{~b}$ \\
\hline \multirow[t]{2}{*}{04073330} & MECAN RIVER & 20 & $\begin{array}{l}\text { SW 1/4 SE 1/4 SEC.1, T. } 16 \text { N.. R.10 E. } \\
\text { MAROUETTE COUNTY, AT BRIDOE ON COUNTY } \\
\text { TRUNK MIGMWAY N, } 5.3 \text { MI }(8.5 \mathrm{KM}) \text { SOUTH } \\
\text { OF NESHKORO. }\end{array}$ & $-\infty$ & $\begin{array}{l}n 8-25-70 \\
09-01-71 \\
07-14-76\end{array}$ & $\begin{array}{l}68.4 \\
92.7 \\
86.2\end{array}$ & 66 & 49 & $b$ \\
\hline & & & WEST BRANCH FONO DU LAC RIVER BASIN & & & & & & \\
\hline 04082804 & $\begin{array}{l}\text { WEST BRANCH } \\
\text { FOND DU LAC }\end{array}$ & RIVER & $\begin{array}{l}\text { SW } 1 / 4 \text { SW } 1 / 4 \text { SEC.4. T.15 N.P R.15 E.. } \\
\text { FOND DU LAC COUNTY, ON COUNTY TRUNK } \\
\text { HIGHWAY M, } 2.6 \mathrm{MI}(4.2 \mathrm{KM}) \text { WEST OF } \\
\text { ROSENDALE. }\end{array}$ & .75 & $\begin{array}{l}11-16-71 \\
11-15-73\end{array}$ & $\begin{array}{l}0.00 \\
0.00\end{array}$ & 0.0 & 0.0 & $\mathrm{~b}$ \\
\hline 04082813 & $\begin{array}{l}\text { WEST BRANCH } \\
\text { FOND DU LAC }\end{array}$ & RIVER & $\begin{array}{l}\text { NW } 1 / 4 \text { SW } 1 / 4 \text { SEC. } 36 . \text { T.16 N., R. } 15 \text { E.. } \\
\text { FOND DU LAC COUNTY. AT CULVERTS ON } \\
\text { COUNTY TRUNK HIGHWAY OOO. AT SEWAGE } \\
\text { PLANT, O.9 MI }(1.4 \mathrm{KM}) \text { NORTHEAST OF } \\
\text { ROSENDALE. }\end{array}$ & 5.32 & $09-13-76$ & ${ }^{2} 0.00$ & .03 & 0.0 & $\mathrm{~b}$ \\
\hline 04082846 & $\begin{array}{l}\text { WFST BRANCH } \\
\text { FOND DU LAC } \\
\text { TRIBUTARY }\end{array}$ & RIVER & $\begin{array}{l}\text { NE 1/4 NW 1/4 SEC.26, T.16 N., R.15 E. } \\
\text { FOND DU LAC COUNTY, AT BRIDGE ON STATE } \\
\text { HIGHWAY } 26,2.0 \mathrm{MI} \text { (3.? KM) NORTH OF } \\
\text { ROSENDALE. }\end{array}$ & 30.5 & $\begin{array}{l}11-17-71 \\
11-14-73 \\
07-13-76\end{array}$ & $\begin{array}{r}3.01 \\
2.04 \\
.19\end{array}$ & $\mathrm{a}$ & $a$ & \\
\hline 04082851 & $\begin{array}{l}\text { TRIBUTARY TO } \\
\text { WEST BRANCH } \\
\text { FOND DU LAC } \\
\text { TRIBUTARY }\end{array}$ & RIVER & $\begin{array}{l}\text { SE 1/4 NF } 1 / 4 \text { SEC.23, T.16 N., R.15 E.. } \\
\text { FOND DU LAC COUNTY, AT CUL VERT ON } \\
\text { PRIVATE ROAD, } 2.8 \text { MI }(4.5 \mathrm{KM}) \text { NORTH OF } \\
\text { ROSENDALE. }\end{array}$ & .96 & $\begin{array}{l}11-18-71 \\
11-14-73\end{array}$ & 0.25 & 0.0 & 0.0 & $\mathrm{~b}$ \\
\hline 04082866 & $\begin{array}{l}\text { WEST BPANCH } \\
\text { FOND DU LAC } \\
\text { TRIBUTARY }\end{array}$ & RIVF.R & $\begin{array}{l}\text { NE } 1 / 4 \text { SE } 1 / 4 \text { SEC.36, T.16 N., R.15 E. } \\
\text { FOND DU LAC COUNTY, AT BRIDGE ON COUNTY } \\
\text { TRUNK HIGHWAY OOO, } 1.5 \mathrm{MI}(2.4 \mathrm{KM}) \text { EAST } \\
\text { OF ROSENDALE. }\end{array}$ & ค. 80 & $\begin{array}{l}11-16-71 \\
11-13-73 \\
07-13-76 \\
08-24-76\end{array}$ & $\begin{array}{l}1.18 \\
.34 \\
0.0 \\
0.0\end{array}$ & 0.0 & 0.0 & $b$ \\
\hline 04082871 & $\begin{array}{l}\text { WEST RRANCH } \\
\text { FOND DU LAC }\end{array}$ & RIVFR & $\begin{array}{l}\text { SE } 1 / 4 \text { SW } 1 / 4 \text { SEC.29. T.16 N.. R.16 E.P. } \\
\text { FOND DU LAC COUNTY NEAR END OF PRIVATE } \\
\text { POAD, } 3.3 \text { MI }(5.3 \mathrm{KMI} \text { EAST OF } \\
\text { ROSENDALE. }\end{array}$ & $56 \cdot 6$ & $\begin{array}{l}11-16-71 \\
11-14-73 \\
07-13-76 \\
08-24-76\end{array}$ & $\begin{array}{l}6.06 \\
4.69 \\
.30 \\
0.0\end{array}$ & 0.0 & 0.0 & $\mathrm{~b}$ \\
\hline 04082876 & $\begin{array}{l}\text { WEST BRANCH } \\
\text { FOND DU LAC } \\
\text { TRIBUTARY }\end{array}$ & RIVFA & $\begin{array}{l}\text { SH } 1 / 4 \text { SW } 1 / 4 \text { SEC.16. T. } 16 \text { N., R, } 16 \text { E.. } \\
\text { FONO D(I LAC COUNTY. AT CULVERT ON } \\
\text { COUNTY TRUNK HIGHWAY N, } 4.8 \text { MI }(7.7 \mathrm{KM}) \\
\text { NORTHEAST OF ROSENDALE. }\end{array}$ & .10 & $\begin{array}{l}11-17-71 \\
11-14-73\end{array}$ & $\begin{array}{l}0.00 \\
0.00\end{array}$ & 0.0 & 0.0 & $\mathrm{~b}$ \\
\hline 04082891 & $\begin{array}{l}\text { TRIBUTARY TO } \\
\text { WEST BRANCH } \\
\text { FOND DU LAC } \\
\text { TRIBUTARY }\end{array}$ & RIVED & $\begin{array}{l}\text { SE } 1 / 4 \text { SW } 1 / 4 \text { SEC.20, T.16 N.PR.16 E., } \\
\text { FOND DU LAC COUNTY. ON COUNTRY ROAD, } \\
3.8 \mathrm{MI}(6.1 \text { KM) NORTHEAST OF ROSENDALE. }\end{array}$ & .43 & $\begin{array}{l}11-17-71 \\
11-15-73\end{array}$ & $\begin{array}{l}0.00 \\
0.00\end{array}$ & 0.0 & 0.0 & $\mathrm{~b}$ \\
\hline 04082891 & $\begin{array}{l}\text { WEST BRANCH } \\
\text { FOND DU LAC } \\
\text { TRIBUTADY }\end{array}$ & PIVEA & $\begin{array}{l}\text { NE } 1 / 4 \text { NW } 1 / 4 \text { SEC.16, T.16 N.P R.16 F., } \\
\text { FOND DII LAC COUNTY, ON COUNTRY ROAO, } \\
\text { S.3 MI }(8.5 \text { KM) NORTHEAST OF ROSENDALE. }\end{array}$ & ?.Qn & $\begin{array}{l}11-17-71 \\
11-15-73\end{array}$ & $\begin{array}{l}0.00 \\
<.05\end{array}$ & 0.0 & 0.0 & $\mathrm{~b}$ \\
\hline 04082896 & $\begin{array}{l}\text { WEST BPANCH } \\
\text { FOND DU LAC } \\
\text { TRIBUTARY }\end{array}$ & RIVER & $\begin{array}{l}\text { SW } 1 / 4 \text { SE } 1 / 4 \text { SEC.16, T.16 N., R.16 E., } \\
\text { FOND DU LAC COUNTY, ON COUNTY TRUNK } \\
\text { HIGHWAY N, } 5.0 \mathrm{MI} \text { (R.0 KM) NORTHEAST OF } \\
\text { ROSENDALE. }\end{array}$ & 1.55 & $\begin{array}{l}11-17-71 \\
11-14-73\end{array}$ & $\begin{array}{l}0.00 \\
0.00\end{array}$ & 0.0 & 0.0 & $\mathrm{~b}$ \\
\hline $04 \cap 83000$ & $\begin{array}{l}\text { WEST RRANCH } \\
\text { FOND DIJ LAC }\end{array}$ & RIVER $^{3}$ & $\begin{array}{l}\text { NE } 1 / 4 \text { NF } 1 / 4 \text { SEC. } 20, \text { T. } 15 \text { N.P R.17 E.. } \\
\text { FOND DU LAC COUNTY, ON COUNTY TRUNK } \\
\text { HIGHWAY T. } 2.0 \text { MI }(3.2 \text { KM) SOUTHWEST OF } \\
\text { DOWNTOWN FOND OU LAC. }\end{array}$ & $\mathrm{R}_{4} \cdot 5$ & (6) & 40.00 & 0.0 & 0.0 & $b$ \\
\hline
\end{tabular}


TABLE 3.--BASIN PROJECT SITE LOCATIONS ANO LOW-FLOW CHARACTERISTICS

UPPER FOX RIVER BASIN

\begin{tabular}{|c|c|c|c|c|c|c|c|c|c|}
\hline \multirow[t]{2}{*}{$\begin{array}{l}\text { STATION } \\
\text { NO. }\end{array}$} & \multirow[t]{2}{*}{ STREAM NAME } & \multirow[t]{2}{*}{$\begin{array}{l}\text { PROJECT } \\
\text { NO. }\end{array}$} & \multirow[b]{2}{*}{ EAST BRANCH FOND DU LAC RIVER BASIN } & \multirow[t]{2}{*}{$\begin{array}{l}\text { ORAIN- } \\
\text { AGE } \\
\text { AREA } \\
\text { (YI } 2 \text { ) }\end{array}$} & \multirow[t]{2}{*}{ DATE } & \multirow[t]{2}{*}{$\begin{array}{l}\text { OIS- } \\
\text { CHARGE } \\
\text { (FT3/S) }\end{array}$} & \multicolumn{2}{|c|}{$\begin{array}{l}\text { CHARAC- } \\
\text { TERISTICS } \\
\text { OT,2 } Q 7,10 \\
\left(F T^{3} / S\right)\left(F T^{3} / 5\right)\end{array}$} & \multirow[t]{2}{*}{$\begin{array}{l}\text { LEVEL } \\
\text { SEIO } \\
\text { (PER- } \\
\text { CENT) }\end{array}$} \\
\hline & & & & & & & & & \\
\hline 04083035 & $\begin{array}{l}\text { EAST BRANCH } \\
\text { FONO DU LAC RIVER }\end{array}$ & 13 & $\begin{array}{l}\text { SE } 1 / 4 \text { NE } 1 / 4 \text { SEC.9, T.14 N., R.16 E., } \\
\text { FONB DU LAC COUNTY, ON COUNTRY ROAO, } \\
8.5 \text { MI }\{13.7 \text { KMI SOUTHWEST OF POST } \\
\text { OFFICE AT FOND DU LAC. }\end{array}$ & 8.55 & $11-17-71$ & 0.00 & 0.0 & 0.0 & $\mathrm{~b}$ \\
\hline 04083075 & CAMPGROUND CREEK & 15 & $\begin{array}{l}\text { NW } 1 / 4 \text { NE } 1 / 4 \text { SEC.29, T.14 N., R.17 E., } \\
\text { FONO DU LAC COUNTY, ON COUNTY TRUNK } \\
\text { HIGHWAY F, } 8.3 \text { MI }(13.4 \text { KM) SOUTH OF } \\
\text { POST OFFICE AT FOND DU LAC. }\end{array}$ & .46 & $11-16-71$ & 0.00 & 0.0 & 0.0 & $b$ \\
\hline 04083095 & $\begin{array}{l}\text { CAMPGROUND CREEK } \\
\text { TRIBUTARY }\end{array}$ & 16 & $\begin{array}{l}\text { NW } 1 / 4 \text { SW } 1 / 4 \text { SEC.21, T.14 N.PR.17 E., } \\
\text { FONB DU LAC COUNTY, ON COUNTRY ROAD, } \\
7.9 \text { MI (12.7 KM) SOUTH OF POST OFFICE } \\
\text { AT FOND DU LAC. }\end{array}$ & $\cdot 25$ & $11-16-71$ & 0.00 & 0.0 & 0.0 & $\mathrm{~b}$ \\
\hline 04083115 & $\begin{array}{l}\text { CAMPGROUNO CREFK } \\
\text { TRIBUTARY }\end{array}$ & 17 & $\begin{array}{l}\text { SW 1/4 NW 1/4 SEC.21, T.14 N.P } 17 \text { E., } \\
\text { FOND OU LAC COUNTY ON COUNTRY ROAD, } \\
7.5 \text { MI (12.1 KM) SOUTH OF POST OFFICE } \\
\text { AT FOND DU LAC. }\end{array}$ & $\cdot 26$ & $11-16-71$ & 0.00 & 0.0 & 0.0 & $\mathrm{~b}$ \\
\hline 04083160 & CAMPGROUND CRFEK & & $\begin{array}{l}\text { SE } 1 / 4 \text { SW } 1 / 4 \text { SEC.11, T.14 N.P R.16 E.P } \\
\text { FOND DII LAC COUNTY, JUST UPSTREAM FROM } \\
\text { SEWAGE TREATMENT PLANT, AT OAKFIELD. }\end{array}$ & 9.44 & $09-13-76$ & ${ }^{2} 3.21$ & 2.9 & 1.8 & 36 \\
\hline 04083195 & $\begin{array}{l}\text { SEVENMILE CREEK } \\
\text { TRIBUTARY }\end{array}$ & 22 & $\begin{array}{l}\text { SE 1/4 NW } 1 / 4 \text { SEC.18, T.15 N., R.16 E.. } \\
\text { FOND DU LAC COUNTY, ON COUNTY TRUNK } \\
\text { HIGHWAY C. } 9.3 \text { MI }(15.0 \text { KMI WEST OF } \\
\text { POST OFFICE AT FOND DU LAC. }\end{array}$ & .61 & $11-17-71$ & 0.00 & 0.0 & 0.0 & $b$ \\
\hline 04083215 & $\begin{array}{l}\text { SEVENMILE CREEK } \\
\text { TRIBUTARY }\end{array}$ & 23 & $\begin{array}{l}\text { NE 1/4 SW } 1 / 4 \text { SEC.19, T.15 N., R.16 E., } \\
\text { FOND DU LAC COUNTY, AT BRIDGE ON COUNTY } \\
\text { TRUNK HIGHWAY C. 9, A MI (15.1 KM) WEST } \\
\text { OF POST OFFICE AT FONO DU LAC. }\end{array}$ & $1 \cdot 29$ & $\begin{array}{l}11-17-71 \\
n 6-24-76 \\
07-13-76\end{array}$ & $\begin{array}{l}.66 \\
.32 \\
.14\end{array}$ & .09 & .02 & b \\
\hline 04083250 & SEVENMILE CREEK & & $\begin{array}{l}\text { NW } 1 / 4 \text { NE } 1 / 4 \text { SEC. } 34, \text { T.15 N., R.16 E.. } \\
\text { FOND DIS LAC COUNTY, } \\
\text { AT BRINGE ON STATE HIGHWAY } 103, \\
\text { AT LAMARTINE. }\end{array}$ & 17.5 & $\begin{array}{l}07-18-73 \\
n B-22-73 \\
n 9-24-74 \\
n 6-24-76 \\
n 7-13=76\end{array}$ & $\begin{array}{l}5<.01 \\
5.01 \\
0.0 \\
0.0 \\
0.0\end{array}$ & 0.0 & 0.0 & $\mathrm{~b}$ \\
\hline 04083375 & $\begin{array}{l}\text { EAST BRANCH } \\
\text { FOND DU LAC RIVFR } \\
\text { TRIBUTARY }\end{array}$ & 33 & $\begin{array}{l}\text { NH } 1 / 4 \text { SF } 1 / 4 \text { SEC.5, T.14 N. R.17 E.. } \\
\text { FOND DU LAC COUNTY, ON COUNTY TRUNK } \\
\text { HIGHWAY FFF. } 4.9 \text { MI } 17.9 \text { KM SOUTH OF } \\
\text { POST OFFICE AT FOND DU LAC. }\end{array}$ & 1.03 & $|1-1 x-7|$ & 0.00 & 0.0 & 0.0 & $b$ \\
\hline $04 \cap 87305$ & $\begin{array}{l}\text { FAST BRANCH } \\
\text { FOND DU LAC RIVER } \\
\text { TRIBUTARY }\end{array}$ & 34 & $\begin{array}{l}\text { NE } 1 / 4 \text { SE } 1 / 4 \text { SEC.5, T.14 N.. R.17 E., } \\
\text { FOND DU LAC COUNTY, ON COUNTY TRUNK } \\
\text { HIGHWAY FFF, } 4.7 \text { MI } 17.6 \text { KM) SOUTH OF } \\
\text { POST OFFICE AT FONN DU LAC. }\end{array}$ & 1.24 & $11-16-71$ & 0.00 & 0.0 & 0.0 & $\mathrm{~b}$ \\
\hline 04083475 & $\begin{array}{l}\text { FAST BRANCH } \\
\text { FONO DU LAC RIVFD } \\
\text { TRIBUTARY }\end{array}$ & 41 & $\begin{array}{l}\text { SW 1/4 NE } 1 / 4 \text { SEC. } 34, \text { T. } 15 \text { N. P.15 E.. } \\
\text { FONO DU LAC COLINTY. ON STATE HIGHWAY } \\
135,3.3 \text { MI }(5.3 \text { KM) SOUTH OF POST } \\
\text { OFFICE AT FONO DU LAC. }\end{array}$ & .46 & $11-15-71$ & 0.00 & 0.0 & 0.0 & b \\
\hline $04 \cap 83495$ & $\begin{array}{l}\text { EAST BRANCH } \\
\text { FOND DU LAC RIVFD } \\
\text { TRIBUTARY }\end{array}$ & 42 & $\begin{array}{l}\text { NW 1/4 NE 1/4 SEC.34, T.15 N.9 R.17 E., } \\
\text { FOND OU LAC COUNTY, ON STATE HIGHWAY } \\
135,3.0 \text { MI (4.8 KM) SOUTH OF POST } \\
\text { RFFICE AT FOND DU LAC. }\end{array}$ & .59 & $11-15-71$ & 0.00 & 0.0 & 0.0 & b \\
\hline 04087500 & $\begin{array}{l}\text { EAST BRANCH } \\
\text { FONO DUS LAC RIVER }\end{array}$ & 43 & $\begin{array}{l}\text { NE } 1 / 4 \text { SW } 1 / 4 \text { SEC.22, T. } 15 \text { N. P R.17 E., } \\
\text { FONO DU LAC COUNTY. ON COUNTY TRUNK } \\
\text { HIGHWAY VV. } 1.6 \text { MI (2.S KM) SOUTH OF } \\
\text { POST OFFICE AT FOND DU LAC. }\end{array}$ & 77.9 & (7) & ${ }^{4} 0.00$ & 1.7 & 0.35 & 38 \\
\hline
\end{tabular}


TABLE 3.--DAEIN PROJECT SITE LOCATIONS AND LOW-FLOW CHARACTERISTICS

UPPER FOX RIVER BASIN

\begin{tabular}{|c|c|c|c|c|c|c|c|c|c|}
\hline \multirow[t]{2}{*}{$\begin{array}{l}\text { STATION } \\
\text { NO. }\end{array}$} & \multirow[t]{2}{*}{ STREAM NAME } & \multirow[t]{2}{*}{$\begin{array}{l}\text { PROJECT } \\
\text { NO. }\end{array}$} & \multirow[b]{2}{*}{ DE NEVUE CREEK BASIN } & \multirow[t]{2}{*}{$\begin{array}{l}\text { DRAIN- } \\
\text { AGE } \\
\text { AREA } \\
\text { (MI2) }\end{array}$} & \multirow[t]{2}{*}{ DATE } & \multirow[t]{2}{*}{$\begin{array}{l}\text { DTS- } \\
\text { CHARGE } \\
\left.\text { (FT } T^{3} / S\right)\end{array}$} & \multicolumn{2}{|c|}{$\begin{array}{l}\text { CHARAC- } \\
\text { TERISTICS }\end{array}$} & $\begin{array}{l}\text { RACY } \\
\text { LEVEL } \\
\text { SEIO } \\
\text { (PER- } \\
\text { CENT) }\end{array}$ \\
\hline & & & & & & & & & \\
\hline 04083920 & DENEVUE CREEK & 2 & $\begin{array}{l}\text { NE } 1 / 4 \text { SW } 1 / 4 \text { SEC. } 25, T .15 \text { N.P R.17 E., } \\
\text { FOND DU LAC COUNTY, ON COUNTRY ROAD, } \\
3.0 \text { MI (4.8 KM) SOUTHEAST OF POST } \\
\text { OFFICE AT FOND DU LAC. }\end{array}$ & $5 \cdot 25$ & $11-16-71$ & 0.00 & 0.0 & 0.0 & b \\
\hline 04083935 & $\begin{array}{l}\text { DE NEVEU CREEK } \\
\text { TRIBUTARY }\end{array}$ & & $\begin{array}{l}\text { SE } 1 / 4 \text { NE } 1 / 4 \text { SEC.B, T.14 N., R.18 E. } \\
\text { FOND DU LAC COUNTY, JUST UPSTREAM FROM } \\
\text { SEWAGE TREATMENT PLANT, } 0.3 \mathrm{MI}(4.8 \mathrm{KM}) \\
\text { NORTH OF EDEN. }\end{array}$ & $2 \cdot 85$ & $\begin{array}{l}07-18-73 \\
08-21-73 \\
09-25-74 \\
07-13-76\end{array}$ & $\begin{array}{l}.44 \\
.61 \\
.30 \\
.36\end{array}$ & a & $a$ & \\
\hline \multirow[t]{2}{*}{04084020} & DENEVUE CREEK & 7 & $\begin{array}{l}\text { NE } 1 / 4 \text { NE } 1 / 4 \text { SEC. } 25, \text { T.15 N.. R.17 E.., } \\
\text { FOND DU LAC COUNTY, ON U.S. HIGHWAY } 45 \text {, } \\
3.1 \text { MI (5.0 KM) SOUTHEAST OF POST } \\
\text { OFFICE AT FOND DU LAC. }\end{array}$ & 16.4 & $n 7-13-76$ & ${ }^{2} 1.05$ & .70 & .30 & 40 \\
\hline & & & NEENAH SLOUGH BASIN & & & & & & \\
\hline 04084417 & $\begin{array}{l}\text { FOX RIVER } \\
\text { TRIBUTARY }\end{array}$ & 1 & $\begin{array}{l}\text { NH 1/4 SH } 1 / 4 \text { SEC. } 28, T^{\top} 20 \text { N. R.17 E. } \\
\text { WINNEBAGO COUNTY, ON STATE HIGHWAY Ii., } \\
\text { AT NEENAH. }\end{array}$ & $19 \cdot 1$ & $\begin{array}{l}08-12-69 \\
08-28-69 \\
11-16-71 \\
07-09-76\end{array}$ & $\begin{array}{r}3.17 \\
3.19 \\
4.19 \\
.88\end{array}$ & .47 & .14 & b \\
\hline
\end{tabular}

\footnotetext{
${ }^{1}$ LOW-FLOW PARTIAL-RECORD STATION.

${ }^{2}$ MINIMUM MEASURED

${ }^{3}$ GAGING STATION.

MINIMUM RECORDED.

${ }^{5}$ ESTIMATE.

${ }^{6}$ NO FLOW FOR MANY DAYS IN MOST YEARS.

701-17-40 T0 01-29-40,01-03-49, 10-12-49, 7-12-50.
} 
MOLF AIVER BASIN

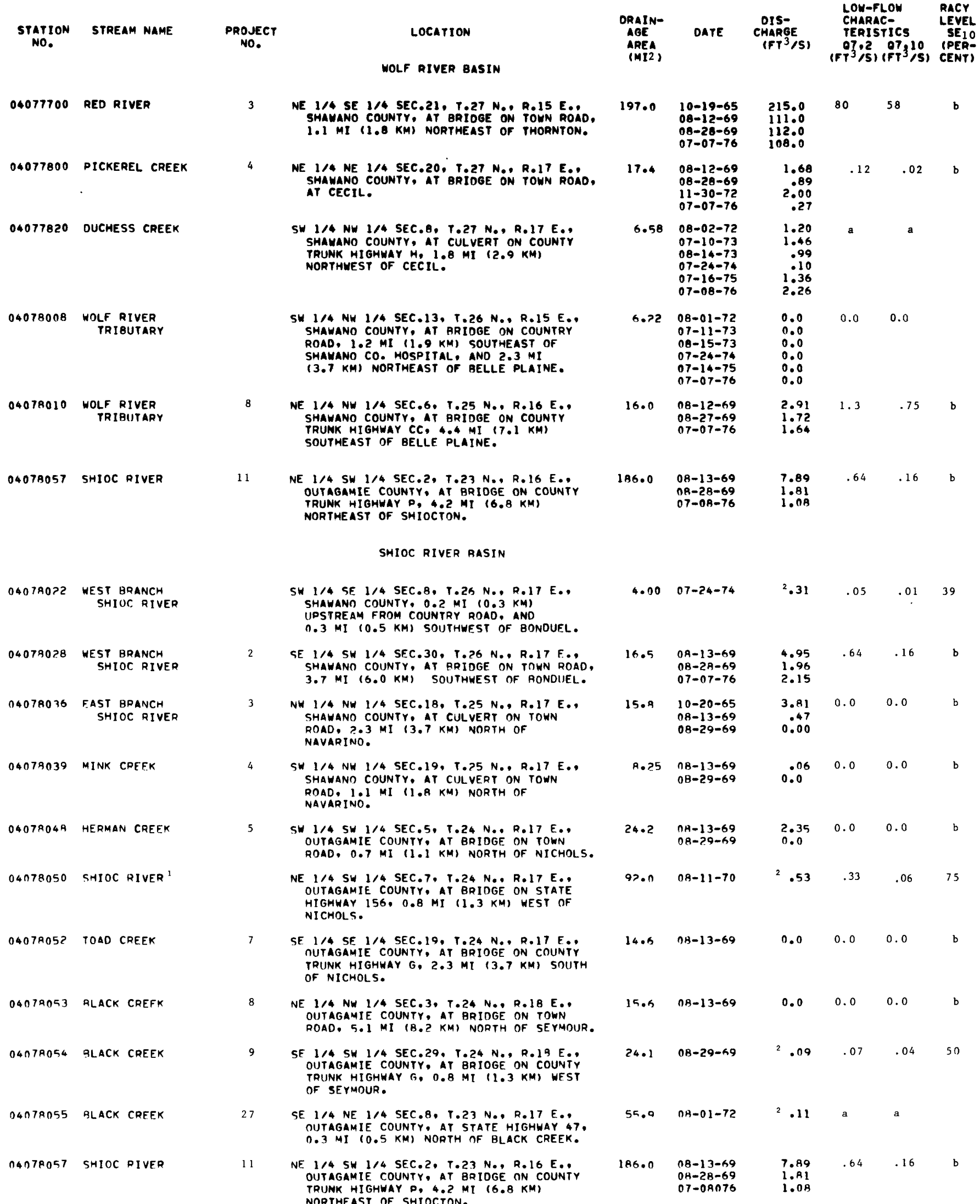


STATION STREAM NAME PROJECY LOCATIDN

EMBARRASS RIVER BASIN

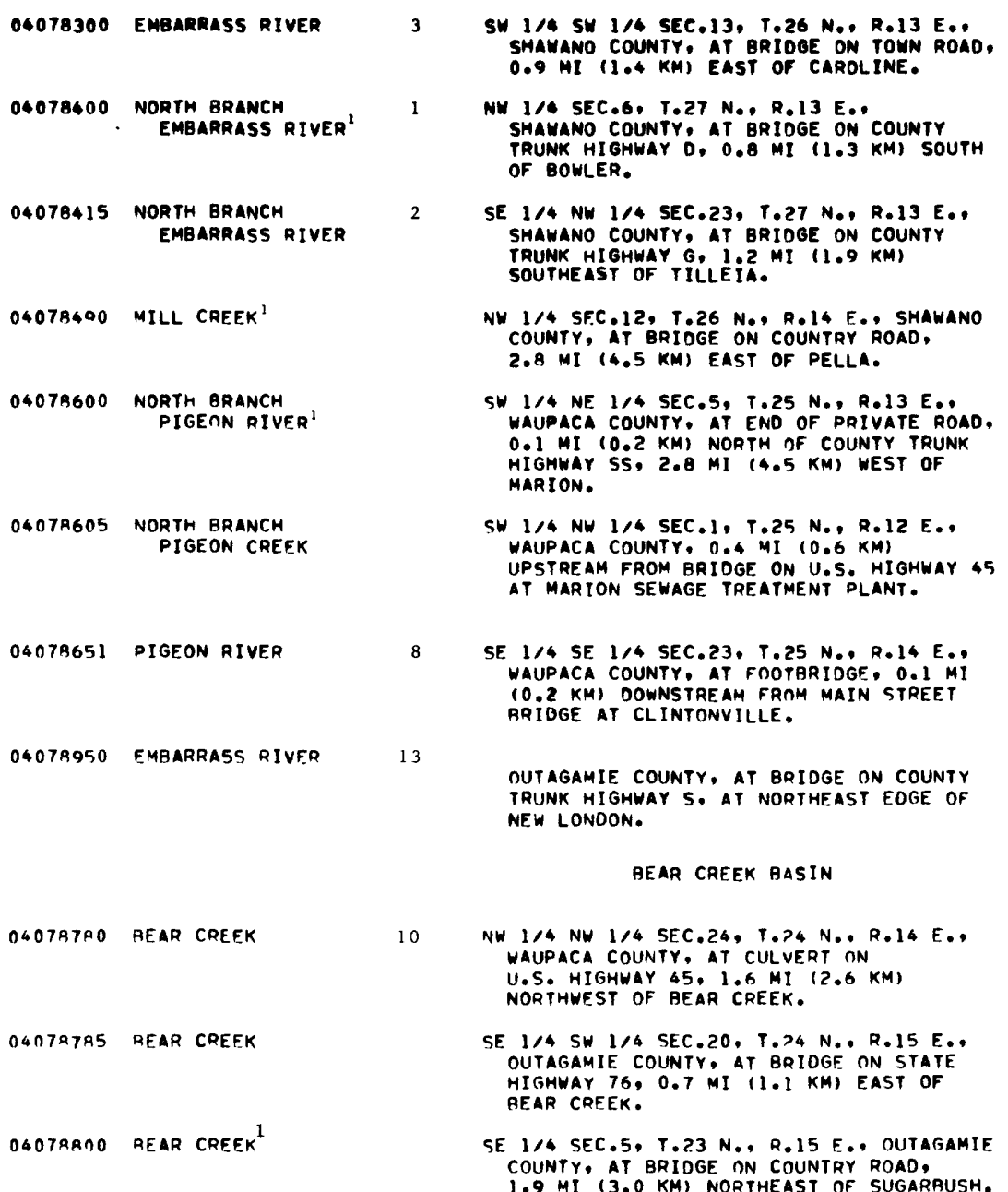

\begin{tabular}{|c|c|c|c|c|c|}
\hline 265.0 & $07-07-76$ & ${ }^{2} 42.4$ & 46 & 26 & 19 \\
\hline 39.5 & $08-06-64$ & 29.16 & 9.8 & 6.0 & 14 \\
\hline $72 \cdot 3$ & $\begin{array}{l}08-26-65 \\
10-19-65 \\
08-11-69 \\
08-27-69\end{array}$ & $\begin{array}{l}15.7 \\
72.1 \\
39.7 \\
28.9\end{array}$ & 14.3 & 8.3 & b \\
\hline 23.1 & $07-07-76$ & ${ }^{2} 7.55$ & 4.9 & 2.5 & 27 \\
\hline 10.8 & $07-07-76$ & ${ }^{2} 2.34$ & 1.6 & .78 & 18 \\
\hline 20.4 & $\begin{array}{l}07-12-73 \\
08-15-73 \\
07-24-74 \\
07-15-75 \\
07-07-76\end{array}$ & $\begin{array}{r}12.0 \\
28.2 \\
7.30 \\
8.20 \\
3.69\end{array}$ & 3.3 & 1.7 & b \\
\hline 109.0 & $08-28-69$ & ${ }^{2} 16.4$ & 16 & 8.1 & 41 \\
\hline 676.0 & $\begin{array}{l}08-12-69 \\
08-28-69 \\
07-09-76\end{array}$ & $\begin{array}{l}296.0 \\
159.0 \\
109.0\end{array}$ & 92 & 50 & $\mathrm{~b}$ \\
\hline 10.5 & $\begin{array}{l}0 B-11-69 \\
08-28-69 \\
07-n 8-76\end{array}$ & $\begin{array}{l}.25 \\
.18 \\
.20\end{array}$ & a & a & \\
\hline 15.3 & $07-07-76$ & ${ }^{2} \cdot 17$ & $<.01$ & $<.01$ & 96 \\
\hline 26.3 & $07-07-76$ & 2.10 & .04 & .01 & 22 \\
\hline
\end{tabular}


LOWER FOX RIVER BASIN

\begin{tabular}{|c|c|c|c|c|c|c|c|c|c|}
\hline \multirow[t]{2}{*}{$\begin{array}{l}\text { STATION } \\
\text { NO. }\end{array}$} & \multirow[t]{2}{*}{ STREAM NAME } & \multirow[t]{2}{*}{$\begin{array}{l}\text { PROJECT } \\
\text { NO. }\end{array}$} & \multirow[b]{2}{*}{ LOWER FOX RIVER BASIN } & \multirow[t]{2}{*}{$\begin{array}{l}\text { DRAIN- } \\
\text { AGE } \\
\text { AREA } \\
\text { (MI2) }\end{array}$} & \multirow[t]{2}{*}{ DATE } & \multirow[t]{2}{*}{$\begin{array}{l}\text { OIS- } \\
\text { CHARGE } \\
\left(F^{3} / S\right)\end{array}$} & \multirow{2}{*}{\multicolumn{2}{|c|}{$\begin{array}{l}\text { CHARAC- } \\
\text { TERISTICS } \\
Q 7,2 \text { Q7,10 } \\
\left(F T^{3} / S\right)\left(F T^{3} / S\right)\end{array}$}} & \multirow[t]{2}{*}{$\begin{array}{l}\text { LEVEL } \\
\text { SE } 10 \\
\text { (DER } \\
\text { CENT) }\end{array}$} \\
\hline & & & & & & & & & \\
\hline 04084911 & PLUM CREEK & 6 & $\begin{array}{l}\text { NW 1/4 SW 1/4 SEC.11. T.21 N.. R.19 E., } \\
\text { BROWN COUNTY, AT BRIDGE ON COUNTY TRUNK } \\
\text { HIGWWAY D, } 1.6 \text { MI }(2.6 \mathrm{KM}) \text { SOUTH OF } \\
\text { WRIGHTSTOWN. }\end{array}$ & $20 \cdot 4$ & $\begin{array}{l}08-12-69 \\
08-27-69 \\
07-07-76\end{array}$ & $\begin{array}{l}.76 \\
.23 \\
.03\end{array}$ & .02 & $<.01$ & $\mathrm{~b}$ \\
\hline 04085070 & ASHWAUBENON CREEK ${ }^{1}$ & 10 & $\begin{array}{l}\text { ON COMMON BOUNDARY OF LAND GRANTS } \\
28 \text { AND } 29, \text { T.23 N.P R, } 20 \text { E.P BROWN } \\
\text { COUNTY, AT CULVERTS ON COUNTY TRUNK } \\
\text { HIGHWAY G, } 0.5 \text { MI }(0,8 \text { KM) WEST OF } \\
\text { DE PERE. }\end{array}$ & $25 \cdot 2$ & $08-11-70$ & ${ }^{2}<.01$ & $<.01$ & $<.01$ & 58 \\
\hline 04085076 & $\begin{array}{l}\text { DUTCHMAN CREEK } \\
\text { TRIBUTARY }\end{array}$ & & $\begin{array}{l}\text { SW 1/4 NW 1/4 SEC, } 7 \text {, T.23 N., R.2O E.. } \\
\text { BROWN COUNTY, AT CUL VERT ON COUNTY } \\
\text { TRUWK HIGHWAY GH, } 3.9 \text { MI } 16.3 \text { KMI } \\
\text { NORTHWEST OF POST OFFICE, AT DE PERE. }\end{array}$ & 3.40 & $\begin{array}{l}07-26-72 \\
10-12-74 \\
08-02-73 \\
10-30-75 \\
07-14-76\end{array}$ & $\begin{array}{l}.10 \\
.98 \\
.06 \\
.15 \\
.08\end{array}$ & .02 & $<.01$ & 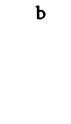 \\
\hline \multirow[t]{2}{*}{04085080} & OUTCHMAN CREEK & 12 & $\begin{array}{l}\text { E 1/2 LAND GRANT } 24, \text { T.23 N.. R.20 E.. } \\
\text { RROWN COUNTY, AT BRIOGE ON COUNTY } \\
\text { TRUNK HIGHWAY HH, } 1.9 \mathrm{MI}(3.1 \mathrm{KM}) \\
\text { NORTH OF DE PERE. }\end{array}$ & $31 \cdot 8$ & $\begin{array}{l}08-13-69 \\
08-28-69 \\
07-08-76\end{array}$ & $\begin{array}{l}.30 \\
.20 \\
.38\end{array}$ & .09 & .01 & $\mathrm{~b}$ \\
\hline & & & EAST RIVER BASIN & & & & & & \\
\hline 04085093 & EAST RIVER & 1 & $\begin{array}{l}\text { NE } 1 / 4 \text { SW } 1 / 4 \text { SEC.G, T.21 N.. R.20 E. } \\
\text { PROWN COUNTYYAT BRIDGF ON TDWN ROAR, } \\
1.5 \mathrm{MI}(2.4 \mathrm{KM}) \text { WEST OF GREFNLEAF. }\end{array}$ & $2 n \cdot 2$ & $\begin{array}{l}08-13-69 \\
08-28-69 \\
08-25-70\end{array}$ & $\begin{array}{r}.89 \\
.16 \\
0.00\end{array}$ & a & a & \\
\hline 04085096 & $\begin{array}{l}\text { EAST RI VER } \\
\text { TRIBUTARY }\end{array}$ & 2 & $\begin{array}{l}\text { NW } 1 / 4 \text { SW } 1 / 4 \text { SEC.6. T.21 N., R.2O E. } \\
\text { RROWN COUNTY AT BRIDGE ON TOWN ROAD, } \\
1.7 \mathrm{MI}(2.7 \mathrm{KM}) \text { WEST OF GREFNLEAF. }\end{array}$ & 7.30 & $\begin{array}{l}n A-13-69 \\
D R-25-70\end{array}$ & $\begin{array}{l}0.00 \\
0.00\end{array}$ & 0.0 & 0.0 & $b$ \\
\hline 04085102 & $\begin{array}{l}\text { EAST RIVFR } \\
\text { TRIRIITARY }\end{array}$ & 3 & $\begin{array}{l}\text { NF. } 1 / 4 \text { SE } 1 / 4 \text { SEC.G. T.21 N. P. RO E.. } \\
\text { RROWN COUNTY AT RRIOGE ON COUNTRY } \\
\text { ROAOQ I.O MI } 1.6 \text { KMI NORTHWEST OF } \\
\text { GREENLEAF. }\end{array}$ & $11 \cdot 4$ & $\begin{array}{l}O A-13-69 \\
O A-28-69 \\
O A-25-70\end{array}$ & $\begin{array}{r}.50 \\
.17 \\
0.00\end{array}$ & a & a & \\
\hline 04045104 & FAST PIVER & 4 & 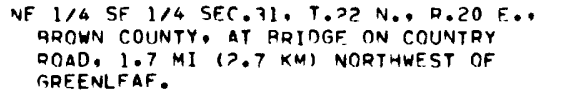 & 37.5 & $n 7-14-76$ & 2.02 & .06 & .01 & a \\
\hline 04085108 & FAST RIVER & 5 & $\begin{array}{l}\text { NE } 1 / 4 \text { NE } 1 / 4 \text { SFC.P0, T.?2 N., R.20 E.. } \\
\text { RROWN COUNTY, AT BRIDGF ON COUNTY TRUNK } \\
\text { HIGHWAY ZZ. } 4.0 \text { MI } 16.4 \mathrm{KM}) \text { NORTH OF } \\
\text { GREENLEAF. }\end{array}$ & 49.8 & $\begin{array}{l}n H-13-69 \\
n A-2 R-69 \\
n R=25=70 \\
10=30-75 \\
C 7-14=76\end{array}$ & $\begin{array}{l}1.74 \\
.27 \\
0.00 \\
1.38 \\
0.0\end{array}$ & 0.0 & 0.0 & b \\
\hline 04045110 & FAST RIVFD ${ }^{1}$ & 6 & $\begin{array}{l}\text { NF 1/4 SF } 1 / 4 \text { SEC.3, T.2? N., R.2O E.. } \\
\text { RROWN COUNTY, AT BRIDGF ON STATE } \\
\text { HIGHWAY 32. 2.5 MI }(4.1 \text { KM) SOUTH OF } \\
\text { nE PERF. }\end{array}$ & $57 \cdot 1$ & $08-25-70$ & ${ }^{2} .07$ & .18 & .06 & 45 \\
\hline 04085110 & EAST RIVER & 7 & $\begin{array}{l}\text { ON LAND GRANT 33, T.23 N., R.?O E.. RROWN } \\
\text { COUNTY, AT HRIDGE ON COUNTY TRIINK } \\
\text { HIGHWAY G, } 1.7 \text { MI }(2.7 \mathrm{KMI} \text { SOUTHEAST OF } \\
\text { DE PERE. }\end{array}$ & 65.1 & $\begin{array}{l}0 R-13-69 \\
08-28-69 \\
08-25-70\end{array}$ & $\begin{array}{r}2.19 \\
.40 \\
.14\end{array}$ & .35 & .17 & b \\
\hline $0408=175$ & HAIRO CREFK & 10 & $\begin{array}{l}\text { NW } 1 / 4 \text { SW } 1 / 4 \text { SEC.33. T.?4 N. P.21 E. } \\
\text { RROWN COUNTY, AT BPIDGE ON COUNTY TRUNK } \\
\text { HIGHWAY CC (DUNZ AVENUE), NEAR EAST } \\
\text { CITY LTMITS, AT GREFN RAY. }\end{array}$ & 23.9 & $\begin{array}{l}B R-13-69 \\
C R-2 A-69 \\
C A-25-70\end{array}$ & $\begin{array}{l}.39 \\
.08 \\
.04\end{array}$ & .08 & .05 & $\mathrm{~b}$ \\
\hline
\end{tabular}


THIN-KEWAUNEE RIVER GASIN AND DOOR COUNTY

\begin{tabular}{|c|c|c|}
\hline $\begin{array}{l}\text { STATION } \\
\text { NOE. }\end{array}$ & STREAM NAME & $\begin{array}{c}\text { PAOJECT } \\
\text { NO. }\end{array}$ \\
\hline 04085192 & $\begin{array}{l}\text { LAKE MICHIGAN } \\
\text { TRIBUTARY }\end{array}$ & \\
\hline 04085193 & $\begin{array}{l}\text { KEWAUNEE RIVER } \\
\text { TRIBUTARY }\end{array}$ & 1 \\
\hline 40951931 & $\begin{array}{l}\text { KEWAUNEE RIVFR } \\
\text { TRIBUTARY }\end{array}$ & $1 \mathrm{a}$ \\
\hline 40951943 & KEWAUNEE RIVER & 5 \\
\hline
\end{tabular}

40951947 SCHOOL CRFEK

04085195 SCHOOL CREEK

40951955 LUXEMRURG CRFEK

40851967 CASCO CREEK

DUDA5197 CASCO CREEK

04085198 SCARBORO CRFEK

40851983 SCARBORO CREFK 40R51987 LITTLE SCARBORO
CREEK

OA OHF190 KEWAUNEE RIVFR

04DRG?חD KEWAUNEE RIVFR ${ }^{3}$
NE 1/4 NE 1/4 SEC.20, T.24 N. R R.25 E.,
KEWAUNEE COUNTY, AT BRIDGE ON CDUNTRY ROAO, 1.0 MI $11.6 \mathrm{KM})$ EAST OF ALASKA. KEWAUNEE COUNTY, ON COUNTRY ROAD $2.9 \mathrm{MI}(4.7 \mathrm{KM})$ NORTHWEST OF LUXEMBURG.

NE 1/4 NW 1/4 SEC.4, T.24 N., R.23 E., KEWAUNEE COUNTY, ON COINTY TRUNK HIGMWAY $K, 3.5 \mathrm{MI}(5.6 \mathrm{kM})$ NORTH OF LUXEMBURG.

NW 1/4 NW 1/4 SEC.11, T.?4 N.. R.23 E.. KEWAUNFE COUNTY, NEAR RRIDGE ON TOWN KEWAUNEE COUNTY, NEAR RRIDGE ON TOWN
POAD, 2.5 MI $14.0 \mathrm{KM})$ NORTHEAST OF POAD, 2.5
LUXEMBURG.

NW 1/4 NW 1/4 SEC.20, T.24 N.. R.23 E.. KEWAINEE COUNTY, ON STATE HIGHWAY 54 , $2.0 \mathrm{MI}(3.2 \mathrm{KM})$ WEST OF LUXEMBIJRG.

SW 1/4 NW 1/4 SEC.15, T.24 N., R.23 E.. KEWAUNEE COUNTY, ON COUNTY TRUNK HIGHWAY A, 1.2 MI $(1.9 \mathrm{KW})$ NORTH OF LIXXEMBIJRG 。

NW 1/4 NF 1/4 SEC.22, T.24 N.. R.23 E.. KE.WAUNEE COUNTY, AT ARIOGE ON COUNTRY KE.WAUNE.E COUNTY, AT ARIOGE ON COUNTRY ROADQ O.7
LUXEMRIJRG

NE $1 / 4$ NW $1 / 4$ 5EC. $17, T .24$ N.. R.24 E., KEWAUNFE COUNTY, AT BRIDGE ON COUNTY hIgHWAY C, AT CASCD.

SE. $1 / 4$ SW $1 / 4$ SEC.18, T.P4 N.. R.24 E.. KEWAUNEE COUNTY, ON COINTRY ROAO. $1.2 \mathrm{MI}(1.9 \mathrm{KM})$ SOUTHWEST OF CASCO.

11 SW 1/4 SW $1 / 4$ SEC.39, T.24 N.. R.23 E.. KEWAIJNFE COUNTY, ON COINTRY ROAD. KEWAINAE COUNTY, ON COIJNTRY ROAD

12 NE $1 / 4$ SE $1 / 4$ SEC. $25, T .24$ N.P. R.23 E.. KEWAUNEE COUNTY. ON COIINTY TRUNK KEWAUNEE COUNTY ON COINNTY TRUNK
HISHWAY A. $3.1 \mathrm{MI}(5.0 \mathrm{KM})$ SOUTHFAST OF HI GHWAY A.

NW 1/4 NE $1 / 4$ SEC. $31, T .24 \mathrm{~N}, ., R_{0} 24 \mathrm{E} .0$ KEWAINEE COUNTY, ON COINTYY TRUNK HIGHWAY A, 4.2 MI $(6.8 \mathrm{KM})$ SOUTHEAST OF LUXEMRIIRG.

14 SW 1/4 SE 1/4 SEC.?2, T.?4 N., R.24 E.. KEWAUNEE COUNTY, ON COIJNTY TRUNK HIGHWAY $C, 5.1$ MI $(9.2 \mathrm{KM})$ EAST OF HUXEMRURG.

SE $1 / 4$ SW $1 / 4$ SEC.14, T. 23 N.. R.24 E.. KFWAUNEE COUNTY, ON LEFY RANK JUST KF.WAUNEE COUNTY, ON LEFY RANK JUST DOWNSTREAM FROM HRIDTE ON COUNTY TRUNK HIGHWAY F, $2.3 \mathrm{MI}(3.7 \mathrm{KM})$ WEST OF KFWAUNEE. ABOUT $7.0 \mathrm{MI} 111.3 \mathrm{KM}$ UPSTREAM FROM MOUTH.
SW 1/4 SE 1/4 SEC.5, T.24 N.. R.23 E.

DRAIN-
AGE
AREA DATE
(MI 2 )
CHARACTERISTICS SE RACY (FT $\left.T^{3} / 5\right)\left(F T^{3} / S\right)$ ( CENT)

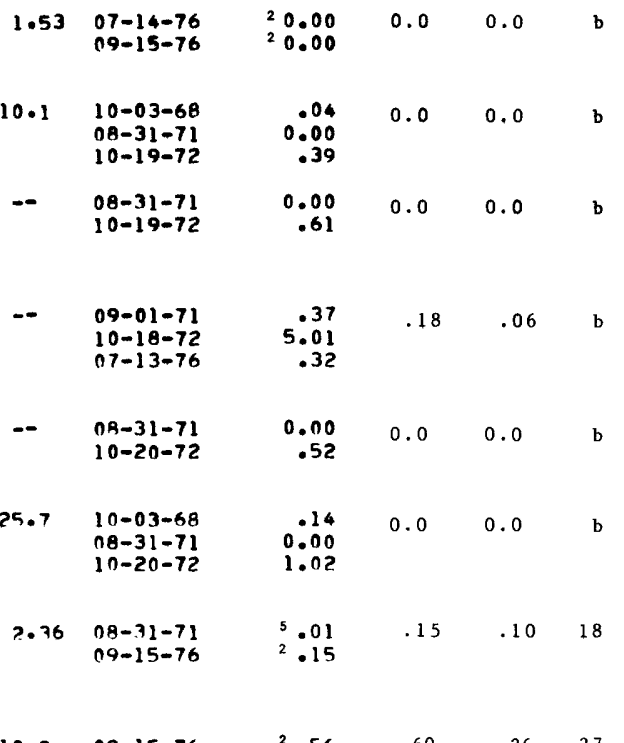

$10.7 \quad 09-15-76$

$-\quad n 9-01-7$ $10-15-72$ $07-13-76$

16.h $\quad 10-03-68$ $10-03-31-71$
$10-18-72$

-- $\quad 09-01-71$ $09-01-71$
$10-18-72$ $10-18-72$
$07-13-76$

n9-01-71 $10-17-72$ $10-17-72$
$07-13-76$

$09-01-71$

$10-17-72$ $10-17-72$
$07-13-76$

$11-03-66$ $11-03-66$
$11-04-66$
2.56

1.1

6.18

$1 \cdot 21$

1.21
1.28
2.60

2.32
4.78

2.28

1.44
2.55
1.46

1.46

$10 . \mathrm{H}$

10.8
32.6
11.8

44.5
44.5
10.0

$10.0 \quad 6.9 \quad 13$

MINIMUM MEASURED

${ }^{3}$ GAGING STATION.

MINIMUM RECORDED

${ }^{5}$ ESTIMATE. 
TABLE 7.--BASIN PROJECT SITE LOCATIONS AND LOW-FLOW CHARACTERISTICS

DES PLAINES AND PIKE RIVER BASIN

\begin{tabular}{|c|c|c|c|c|c|c|c|c|c|}
\hline \multirow[t]{2}{*}{$\begin{array}{l}\text { STATION } \\
\text { NO. }\end{array}$} & \multirow[t]{2}{*}{ STREAM NAME } & \multirow[t]{2}{*}{$\begin{array}{l}\text { PROJECT } \\
\text { NO. }\end{array}$} & \multirow[b]{2}{*}{ PIKE RIVER BASIN } & \multirow[t]{2}{*}{$\begin{array}{l}\text { DRAIN- } \\
\text { AGE } \\
\text { AREA } \\
\text { (MI } 2 \text { ) }\end{array}$} & \multirow[t]{2}{*}{ DATE } & \multirow[t]{2}{*}{$\begin{array}{l}\text { DIS- } \\
\text { CHARGE } \\
\left.\text { (FT }^{3} / S\right)\end{array}$} & \multirow{2}{*}{\multicolumn{2}{|c|}{$\begin{array}{l}\text { CHARAC- } \\
\text { TERISTICS } \\
07,2 \text { OT,10 } \\
(F T 3 / S)\left(F T^{3} / 5\right)\end{array}$}} & \multirow[t]{2}{*}{$\begin{array}{l}\text { RACY } \\
\text { LEVEL } \\
\text { SE } 10 \\
\text { (PER } \\
\text { CENT) }\end{array}$} \\
\hline & & & & & & & & & \\
\hline 04087243 & PIKE RIVER & 10 & $\begin{array}{l}\text { SE } 1 / 4 \text { NW } 1 / 4 \text { SEC } 14, \text { T.3 N., R.22 E., } \\
\text { RACINE COUNTY AT CULVERT ON NIGHWAY } \\
20,4.4 \text { MI } 17.1 \text { KMI WEST OF RACINE. }\end{array}$ & 4.42 & $09-19-74$ & 2.03 & $<.01$ & $<.01$ & 18 \\
\hline 04087244 & $\begin{array}{l}\text { PIKE RIVER } \\
\text { TRIBUTARY }\end{array}$ & 11 & $\begin{array}{l}\text { SW } 1 / 4 \text { NE } 1 / 4 \text { SEC } 21 \text { T T.3 N. R. } 22 \text { E., } \\
\text { RACINE COUNTY, AT CULVERT ON FANCHER } \\
\text { ROAD (COUNTY TRUNK HIGHWAY H). } 0.3 \mathrm{MI} \\
\text { (0.5 KM) NORTH OF STURTEVANT. }\end{array}$ & .65 & $\begin{array}{l}07-17-73 \\
08-08-73 \\
10-26-73 \\
n 9-19-74 \\
09-18-75\end{array}$ & $\begin{array}{l}20.00 \\
20.00 \\
20.00 \\
20.00 \\
20.00\end{array}$ & 0.0 & 0.0 & $\mathrm{~b}$ \\
\hline 40872465 & $\begin{array}{l}\text { PIKE RIVER } \\
\text { TRIBUTARY }\end{array}$ & 8 & $\begin{array}{l}\text { SE } 1 / 4 \text { SW } 1 / 4 \text { SEC.PT, T.3 N. R.22 E. } \\
\text { RACINE COUNTY, ON PRIVATE ROAO } 0.6 \mathrm{MI} \\
11.0 \text { KM }) \text { ABOVE MOUTH. } 1.3 \mathrm{MI}(2.1 \mathrm{KM}) \\
\text { SOUTHEAST OF STURTEVANT. }\end{array}$ & 1.10 & $\begin{array}{l}10-25-73 \\
09-19-74\end{array}$ & $\begin{array}{l}<.05 \\
0.00\end{array}$ & 0.0 & 0.0 & $\mathrm{~b}$ \\
\hline 04087247 & $\begin{array}{l}\text { PIKE RIVER } \\
\text { TRIBUTARY }\end{array}$ & 7 & $\begin{array}{l}\text { SE I/4 NF } 1 / 4 \text { SEC.33, T.3 N., R.22 E.P } \\
\text { RACINE COUNTY, ON COUNTY TRUNK HIGHWAY } \\
\text { EA. } 1.5 \text { MI } 12.4 \text { KMI SOUTH OF } \\
\text { STURTEVANT. }\end{array}$ & 1.08 & $\begin{array}{l}10-25-73 \\
09-19-74\end{array}$ & $\begin{array}{r}.43 \\
0.00\end{array}$ & 0.0 & 0.0 & $\mathrm{~b}$ \\
\hline 04087250 & PIKE RIVER ${ }^{3}$ & 18 & $\begin{array}{l}\text { NW } 1 / 4 \text { SW } 1 / 4 \text { SEC. } 27 \text { T.? N., R.22 E., } \\
\text { KENOSHA COUNTY. ON STITE MIGHWAY } 430 \\
3,9 \text { MI }(6.3 \text { KM) NORTHWEST OF ONWNTOWN } \\
\text { KENOSHA. }\end{array}$ & $7 \cdot 35$ & $\begin{array}{l}07-11-63 \\
09-18-63\end{array}$ & $\begin{array}{l}2.01 \\
2.01\end{array}$ & 0.0 & 0.0 & $\mathrm{~b}$ \\
\hline 04087253 & $\begin{array}{l}\text { DIKE RIVER } \\
\text { TRIBUTARY }\end{array}$ & 15 & $\begin{array}{l}\text { SW } 1 / 4 \text { SE } 1 / 4 \text { SEC.9, T.2 N.P R.22 E., } \\
\text { KENOSHA COUNTY. AT CULVERT ON CHICAGO } \\
\text { \& MILWAUKEE R.R., AT NORTH EOGE NF } \\
\text { SOMERS. }\end{array}$ & .93 & $\begin{array}{l}07-17-73 \\
09-19-75\end{array}$ & $\begin{array}{l}0.00 \\
0.00\end{array}$ & 0.0 & 0.0 & $\mathrm{~b}$ \\
\hline 04087255 & $\begin{array}{l}\text { PIKE CREEK } \\
\text { TRIBUTARY }\end{array}$ & 17 & $\begin{array}{l}\text { SW 1/4 SF 1/4 SEC.3, T.2 N., R.22 E., } \\
\text { KENOSHA COUNTY, AT RDINGE ON COUNTY } \\
\text { TRUNK HIGHWAY A, } 1.7 \text { MI } 12.7 \mathrm{KMI} \\
\text { NORTHEAST OF SOMERS. }\end{array}$ & 2.48 & $\begin{array}{l}10-25-73 \\
n 9-19-74\end{array}$ & $\begin{array}{r}.04 \\
0.00\end{array}$ & 0.0 & 0.0 & $\mathrm{~b}$ \\
\hline 04087257 & PIKE RIVER ${ }^{3}$ & 5 & 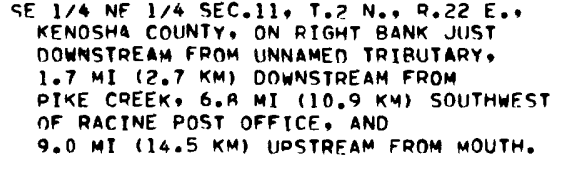 & $3 \AA .7$ & $11-11-71$ & ${ }^{4} .53$ & 0.0 & 0.0 & $\mathrm{~b}$ \\
\hline 04097260 & PIKE RIVER ? & 39 & $\begin{array}{l}\text { NH 1/4 SW } 1 / 4 \text { SEC.6, T.2 N., R.23 E., } \\
\text { KENOSHA COUNTY. ON COUNTY TRUNK HIGHWAY } \\
\text { YE } 5.0 \text { MI }(8.0 \text { KM) NORTH OF DOWNTOWN } \\
\text { KENOSHA. }\end{array}$ & $4 n \cdot 4$ & $09-11-63$ & 2.01 & 0.15 & 0.02 & $\mathrm{~b}$ \\
\hline
\end{tabular}


TABLE B.--BASIN PROJECT SITE LOCATIONS AND LOW-FLOM CHARACTERISTICS

TREMPEALEAU-BUFFALO RIVER BASIN

STATION STREAM NAME

NO.

05379249 PIGEON CREEK
PROJECT

NO.

LOCATION

PIGEON CREEK RASIN

\begin{tabular}{|c|c|}
\hline DRAIN- & DATE \\
\hline
\end{tabular}

AGE

(MI 2)

SW 114 SE 1/4 SEC . 34, T.23 N., R.7 W. TREMPEALEAU COUNTY, $300 \mathrm{FT}(150 \mathrm{M})$ DOWNSTREAM OF DAM IN PIGEON FALLS.

58.4

$\begin{array}{ll}08-10-72 & 31.3 \\ 06-27-73 & 77.1 \\ 09-12-73 & 44.1 \\ 08-20-76 & 25.2\end{array}$

TABLE 9.--AASIN PROJECT SITE LOCATIONS ANO LOW-FLOH CHADACTERISTICS

BLACK RIVER RASIN

\begin{tabular}{|c|c|c|c|}
\hline \multirow[t]{2}{*}{$\begin{array}{l}\text { STATION } \\
\text { NO. }\end{array}$} & STREAM NAME & $\begin{array}{l}\text { PROJECT } \\
\text { ND. }\end{array}$ & LOCATION \\
\hline & & & BEAVER CREEK RASIN \\
\hline $0538>0,0$ & REAVER CDFEK & 13 & 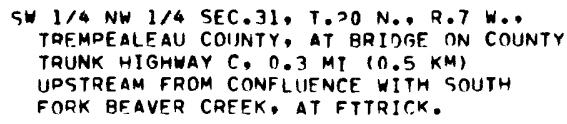 \\
\hline 05382057 & $\begin{array}{l}\text { SOUTH FORK } \\
\text { REAVFR CREFK }\end{array}$ & 2 & 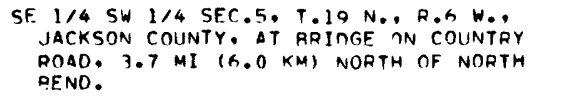 \\
\hline $0538>072$ & GERMAN COULEE & 4 & $\begin{array}{l}\text { NE I/4 SW I/4 SEC.5, T.1Q N. R.6 W. } \\
\text { JACKSON COUNTY. AT RRINGE ONI COUNTRY } \\
\text { ROAD. } 4.1 \mathrm{MI}(6.6 \mathrm{KM}) \text { NORTH OF NORTH } \\
\text { REND. }\end{array}$ \\
\hline $0538 ? 100$ & $\begin{array}{l}\text { SOUTH FORK } \\
\text { REAVER CRFFK }\end{array}$ & 10 & 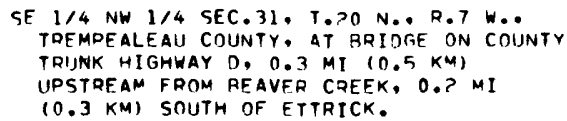 \\
\hline
\end{tabular}

\begin{tabular}{|c|c|c|c|c|c|}
\hline $\begin{array}{l}\text { ORAIN- } \\
\text { AGE } \\
\text { ARE A } \\
\left(M I^{2}\right)\end{array}$ & DATF. & $\begin{array}{l}\text { DIS- } \\
\text { CHARGE } \\
\left(F T^{3} / S\right)\end{array}$ & $\begin{array}{r}\text { LOH-1 } \\
\text { CHAR } \\
\text { TERI } \\
37.2 \\
\text { IFT } 315\end{array}$ & $\begin{array}{l}\text { Ow } \\
- \\
\text { ICS } \\
07: 10 \\
\left(\mathrm{FT}^{3} / \mathrm{S}\right)\end{array}$ & $\begin{array}{l}\text { ACCI)- } \\
\text { RACY } \\
\text { LEVEL } \\
\text { SF } 10 \\
\text { (PFQ- } \\
\text { CENT) }\end{array}$ \\
\hline 51.0 & $08-10-70$ & ${ }^{2} 17.4$ & 14 & 9.2 & 10 \\
\hline-- & $0 H-18-70$ & 0.00 & 0.0 & 0.0 & b \\
\hline-- & $0 R-1 A-70$ & 0.00 & 0.0 & 0.0 & b \\
\hline 37.1 & $08-19-64$ & $24.2 ?$ & 6.1 & 4.4 & 11 \\
\hline
\end{tabular}

${ }^{1}$ LOW-FLOW PARTIAL-RECORD STATION.

MINIMUM MEASURED. 
LOMER MISCONSIN RIVER BASIN

\begin{tabular}{|c|c|c|c|c|c|c|c|c|c|}
\hline \multirow[t]{2}{*}{$\begin{array}{l}\text { STATION } \\
\text { NO. }\end{array}$} & \multirow[t]{2}{*}{ STREAM NAME } & \multirow[t]{2}{*}{$\begin{array}{l}\text { PROJECT } \\
\text { NO. }\end{array}$} & \multirow[b]{2}{*}{ PINE RIVER BASIN } & \multirow[t]{2}{*}{$\begin{array}{l}\text { DRAIN- } \\
\text { AGE } \\
\text { AREA } \\
\text { (MI') }\end{array}$} & \multirow[t]{2}{*}{ DATE } & \multirow[t]{2}{*}{$\begin{array}{l}\text { DIS- } \\
\text { CMARGE } \\
\left(F T^{3} / S\right)\end{array}$} & \multirow{2}{*}{\multicolumn{2}{|c|}{$\begin{array}{l}\text { CHARAC- } \\
\text { TERISTICS } \\
07,207,10 \\
\left(F T^{3} / 5\right)\left(F T^{3} / 5\right)\end{array}$}} & \multirow[t]{2}{*}{$\begin{array}{l}\text { LEVEL } \\
\text { SE } 10 \\
\text { (PER: } \\
\text { CENT) }\end{array}$} \\
\hline & & & & & & & & & \\
\hline $05 \$ 06710$ & PINE RIVER & $36 a$ & $\begin{array}{l}\text { SH } 1 / 4 \text { SW } 1 / 4 \text { SEC.6, T.12 N., R. I E. } \\
\text { RICMLAND COUNTY, AT BRIDGE ON STATE } \\
\text { HIGHWAY BO, AT YUBA. }\end{array}$ & $27 \cdot 2$ & $09-09-74$ & ${ }^{2} 10.5$ & 4.0 & 2.9 & 13 \\
\hline 05406716 & MELANCTHON CREEK & $5 c$ & $\begin{array}{l}\text { NE 1/4 NE 1/4 5EC, } 10.9 \text { T.12 N., R.1 E. } \\
\text { RICHLAND COUNTY, APPROXIMATELY } 500 \text { FT } \\
\text { (150 M) NORTH OF PRIVATE FARM DRIVE, } \\
\text { APPROXIMATELY } 100 \text { FT (30 M) EAST OF } \\
\text { COUNTY TRUNK HIGHWAY C. O.3 MI (O.5 KM) } \\
\text { UPSTREAM FROM MOUTH OF GRINSELL CREEK. }\end{array}$ & 4.66 & $\begin{array}{l}06-03-74 \\
09-09-74 \\
05-14-75\end{array}$ & $\begin{array}{l}3.98 \\
2.97 \\
3.09\end{array}$ & 2.3 & 2.0 & b \\
\hline 05406728 & $\begin{array}{l}\text { WEST BRANCH } \\
\text { PINE RIVER }\end{array}$ & $9 a$ & $\begin{array}{l}\text { NH } 1 / 4 \text { NH } 1 / 4 \text { SEC. } 15 \text {, T } 12 \text { N., R. } 1 \text { W. } \\
\text { RICHLAND COUNTY ABOUT } 500 \text { FT (150 M) } \\
\text { BELOH MOUTH OF BASSWOOD CREEK. } 2.2 \mathrm{MI} \\
\text { (3.5 KM) NORTHWEST OF SLOOM CITY. }\end{array}$ & $10 \cdot 5$ & $\begin{array}{l}06-04-74 \\
09-09-74 \\
05-15-75\end{array}$ & $\begin{array}{l}6.89 \\
5.57 \\
5.87\end{array}$ & 3.2 & 2.5 & $\mathrm{~b}$ \\
\hline 05406730 & $\begin{array}{l}\text { WEST BRANCH } \\
\text { PINE RIVER }\end{array}$ & $9 b$ & 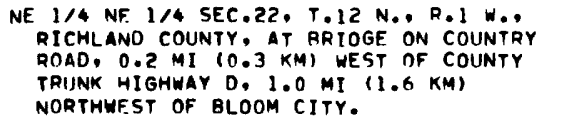 & 13.0 & $\begin{array}{l}06-04-74 \\
09-09-74 \\
05-15-75\end{array}$ & $\begin{array}{l}9.01 \\
8.16 \\
7.75\end{array}$ & 4.0 & 3.2 & $\mathrm{~b}$ \\
\hline 05406732 & $\begin{array}{l}\text { WEST BRANCH } \\
\text { PINE RIVER }\end{array}$ & 10 & $\begin{array}{l}\text { NE } 1 / 4 \text { NH } 1 / 4 \text { SEC. } 26, \text { T.12 N. OR.1 W. } \\
\text { RICHLANO COUNTY, AT BRIDGE ON COUNTY } \\
\text { TRUNK HIGHWAY H. AT BLOOM CITY. }\end{array}$ & $14 \cdot 6$ & $\begin{array}{l}08-19-70 \\
10-17-72 \\
06-04-74\end{array}$ & $\begin{array}{l}5.85 \\
8.89 \\
10.8\end{array}$ & 4.3 & 3.4 & $\mathrm{~b}$ \\
\hline 05406735 & GAULT HOLLOW CPFFK & $10 \mathrm{a}$ & 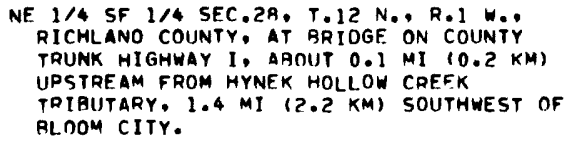 & 2.46 & $\begin{array}{l}06-04-74 \\
09-10-74 \\
05-14-75\end{array}$ & $\begin{array}{l}2.33 \\
2.48 \\
2.33\end{array}$ & a & a & \\
\hline 05406737 & HYNEK HOLLOW CREEK & $10 \mathrm{~b}$ & 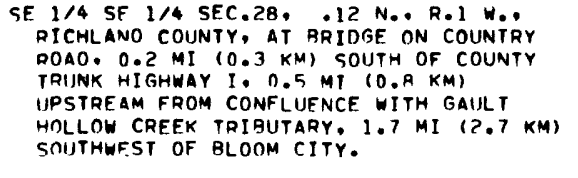 & 2.59 & $\begin{array}{l}06-04-74 \\
09-10-74 \\
05-14-75\end{array}$ & $\begin{array}{l}1.57 \\
1.42 \\
1.55\end{array}$ & .74 & .60 & b \\
\hline 05406740 & $\begin{array}{l}\text { WEST BPANCH } \\
\text { PINE RIVER } \\
\text { TRIBUTARY }\end{array}$ & $11 \mathrm{~b}$ & $\begin{array}{l}\text { NW } 1 / 4 \text { SW } 1 / 4 \text { SEC. } 26 \text {. T.12 N. R R.1 W., } \\
\text { RICHLAND COUNTY, AT RRIDGE ON COINTY } \\
\text { TRIINK HIGHWAY H. } 0.5 \text { MI (D. R KM) SOUTH } \\
\text { OF BLOOM CITY. }\end{array}$ & 6.12 & $\begin{array}{l}10-17-72 \\
06-04-74 \\
09-10-74 \\
15-14-75\end{array}$ & $\begin{array}{l}4.16 \\
5.29 \\
4.40 \\
4.96\end{array}$ & 2.4 & 1.8 & $\mathrm{~b}$ \\
\hline 05406743 & $\begin{array}{l}\text { WEST BRANCH } \\
\text { PINE RIVER }\end{array}$ & 12 & $\begin{array}{l}\text { NE. } 1 / 4 \text { NE } 1 / 4 \text { SEC. } 9 \text {, T.1I N.. R.1 E.P } \\
\text { RICHLAND COUNTY, AT PRIDGE ON COUNTY } \\
\text { TRUNK HIGHWAY } 0,0.5 \text { MI }(0 . \text { K KM) WFST } \\
\text { OF ROCKBRIDGE. }\end{array}$ & 37.6 & $\begin{array}{l}08-19-70 \\
10-18-72 \\
06-03-74\end{array}$ & $\begin{array}{l}16.4 \\
20.3 \\
30.7\end{array}$ & 12 & 9.4 & $b$ \\
\hline 05406745 & DINE RIVEP & 13 & $\begin{array}{l}\text { SE 1/4 NW } 1 / 4 \text { SEC.1OE TIII N., R.I E., } \\
\text { RICHLAND COUNTY AT RRIDGE ON STATE } \\
\text { HIGHWAY BO. AT ROCKRRIDGE. }\end{array}$ & 117 & $\begin{array}{l}08-19-70 \\
10-18-72 \\
n G-03-74\end{array}$ & $\begin{array}{l}48.1 \\
76.2 \\
102\end{array}$ & 38 & 29 & b \\
\hline 05404750 & DINE RIVER ${ }^{1}$ & 15 & $\begin{array}{l}\text { NW } 1 / 4 \text { SEC.27, T.11 N.. R.1 E. R RICHLAND } \\
\text { COUNTY. AT GRIDGE ON STATE HIGHWY AN. } \\
1.0 \mathrm{MI}(1.6 \mathrm{KM} \text { SOUTH OF BUCK CREEK. }\end{array}$ & 129 & $09-07-67$ & ${ }^{2} 48.8$ & 46 & 37 & 13 \\
\hline 04405752 & FANCY CREEK & $15 \mathrm{a}$ & 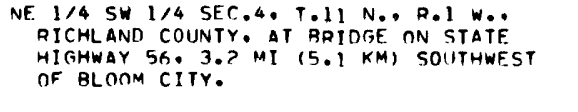 & $4 \cdot 14$ & $\begin{array}{l}n 6-03-74 \\
n 9-09-74 \\
05-15-75\end{array}$ & $\begin{array}{l}1.94 \\
1.52 \\
2.01\end{array}$ & 1.2 & 1.0 & b \\
\hline 05406754 & FANCY CRFFK & $15 \mathrm{~b}$ & $\begin{array}{l}\text { SW 1/4 NF } 1 / 4 \text { SEC.10. T.II N. R.1 W. } \\
\text { RICHLAND COUNTY, AT RRIDGE ON COUNTY } \\
\text { TRUNK HIGHWAY H. 2.? MI } 13.5 \mathrm{KM}) \\
\text { NORTHWEST OF GILLINGHAM. }\end{array}$ & 6.57 & $\begin{array}{l}06-03-74 \\
09-09-74 \\
05-15-75\end{array}$ & $\begin{array}{l}4.11 \\
3.49 \\
4.66\end{array}$ & 2.5 & 2.1 & $\mathrm{~b}$ \\
\hline 05406757 & $\begin{array}{l}\text { FANCY CREEK } \\
\text { TRIBUTARY }\end{array}$ & $16 a$ & $\begin{array}{l}\text { NW } 1 / 4 \text { NF. } 1 / 4 \text { SEC.16, T.11 N.. R.1 W. } \\
\text { PICHLAND COUNTY, AT RRTDGE ON PRIVATE } \\
\text { ORIVE. } 2.7 \text { MI }(4.3 \mathrm{KM}) \text { NORTHWEST OF } \\
\text { GILLINGHAM. }\end{array}$ & 1.59 & $\begin{array}{l}n 6-03-74 \\
n 9-09-74 \\
n 5-15-75\end{array}$ & $\begin{array}{l}.73 \\
.71 \\
.79\end{array}$ & .35 & .28 & $\mathrm{~b}$ \\
\hline 05404758 & $\begin{array}{l}\text { FANCY CREEK } \\
\text { TRIBIJTARY }\end{array}$ & $16 \mathrm{~b}$ & $\begin{array}{l}\text { SW 1/4 NW } 1 / 4 \text { SEC. } 15 \text {, T.11 N., R.I W., } \\
\text { RICHLANO COUNTY, INOFT (30 M) } \\
\text { NORTHEAST ANO ALONG SINE OF COINTRY } \\
\text { ROADE } 2.2 \text { MI }(3.5 \mathrm{KM}) \text { WFST OF } \\
\text { GILLINGHAM. }\end{array}$ & $? \cdot ? 0$ & $\begin{array}{l}06-03-74 \\
09-09-74 \\
05-15-75\end{array}$ & $\begin{array}{r}1.64 \\
.79 \\
.95\end{array}$ & .41 & .30 & $b$ \\
\hline 05406763 & $\begin{array}{l}\text { TRIRUTARY TO } \\
\text { FANCY CRFEK } \\
\text { TRIBUITARY }\end{array}$ & $16 \mathrm{c}$ & 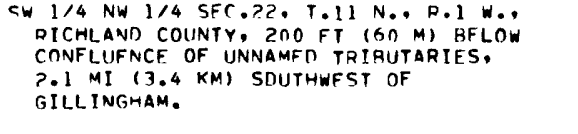 & 2.13 & $\begin{array}{l}06-03-74 \\
09-10-74 \\
05-14-75\end{array}$ & $\begin{array}{l}1.45 \\
1.17 \\
1.49\end{array}$ & .84 & .74 & $\mathrm{~b}$ \\
\hline $054 n \times 7 \times 4$ & $\begin{array}{l}\text { TRIRITTARY TO } \\
\text { FANCY CRFEK } \\
\text { TRIBUTADY }\end{array}$ & $16 \mathrm{~d}$ & $\begin{array}{l}\text { SE } 1 / 4 \text { SE } 1 / 4 \text { SEC. } 15 \text {. T.11 N.. R.1 W.P } \\
\text { RICHLAND COUNTY, AT PRIDGE ON COUNTY } \\
\text { TRUNK HIGHWY Z, } 1.3 \text { MI }(2.1 \text { KM) WFST } \\
\text { OF GILLINGHAM. }\end{array}$ & 2.73 & $\begin{array}{l}06-03-74 \\
09-10-74 \\
05-14-75\end{array}$ & $\begin{array}{l}1.83 \\
1.53 \\
1.82\end{array}$ & 1.2 & 1.1 & b \\
\hline
\end{tabular}




\begin{tabular}{|c|c|c|c|c|c|c|c|c|c|}
\hline \multirow[t]{2}{*}{$\begin{array}{l}\text { STATION } \\
\text { NO. }\end{array}$} & \multirow[t]{2}{*}{ STREAM NAME } & \multirow[t]{2}{*}{$\begin{array}{l}\text { PROJECT } \\
\text { NO. }\end{array}$} & \multirow[b]{2}{*}{ PINE RIVER BASIN--CONTINUED } & \multirow[t]{2}{*}{$\begin{array}{l}\text { DRAIN- } \\
\text { AGE } \\
\text { AREA } \\
\text { (MI } I^{2} \text { ) }\end{array}$} & \multirow[t]{2}{*}{ DATE } & \multirow[t]{2}{*}{$\begin{array}{l}\text { DIS- } \\
\text { CHARGE } \\
\left(F T^{3} / S\right)\end{array}$} & \multirow{2}{*}{\multicolumn{3}{|c|}{ 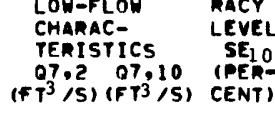 }} \\
\hline & & & & & & & & & \\
\hline 05406780 & FANCY CREEK & 19 & $\begin{array}{l}\text { NE 1/4 SE 1/4 SEC.32, T.11 N., R.1 E., } \\
\text { RICHLAND COUNTY AT BRIDGE ON STATE } \\
\text { HIGHWAYS } 56 \text { AND BO, } 3.5 \text { MI } 15.6 \mathrm{KMI} \\
\text { NORTH OF RICHLAND CENTER. }\end{array}$ & $28 \cdot 4$ & $\begin{array}{l}08-19-70 \\
10-17-72 \\
06-03-74\end{array}$ & $\begin{array}{l}10.7 \\
13.4 \\
20.2\end{array}$ & 8.8 & 7.6 & b \\
\hline 05406781 & HORSE CREEK & $19 \mathrm{a}$ & $\begin{array}{l}\text { NH } 1 / 4 \text { NW } 1 / 4 \text { SEC.1. TIIO N.P R.1 W., } \\
\text { RICMLAND COUNTY, AT BRIDGE ON COUNTRY } \\
\text { ROAB. O.1 MI }(0.2 \text { KM) SOUTH OF COUNTY } \\
\text { TRUNK HIGHWAY A, } 3.5 \mathrm{MI}(5.6 \mathrm{KM}) \text { SOUTH } \\
\text { OF GILLINGHAM. }\end{array}$ & 3.44 & $\begin{array}{l}06-03-74 \\
09-10-74 \\
05-14-75\end{array}$ & $\begin{array}{l}2.50 \\
1.81 \\
2.50\end{array}$ & 1.1 & .93 & $\mathrm{~b}$ \\
\hline 05406782 & $\begin{array}{l}\text { TRIBUTARY TO } \\
\text { HORSE CREEK }\end{array}$ & $19 \mathrm{~b}$ & 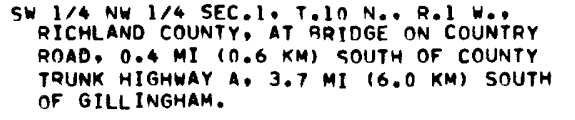 & 1.47 & $\begin{array}{l}06-03-74 \\
09-10-74 \\
05-14-75\end{array}$ & $\begin{array}{r}1.02 \\
.50 \\
.86\end{array}$ & .22 & .15 & $\mathrm{~b}$ \\
\hline 05406723 & HORSE CREEK & $19 \mathrm{c}$ & $\begin{array}{l}\text { SE } 1 / 4 \text { NW } 1 / 4 \text { SEC. } 6 \text {, T.10 N. R.1 E.P } \\
\text { RICHLANO COUNTY, AT BRIDGE ON COUNTRY } \\
\text { ROAD. O.1 MI }(0.2 \text { KM) SOUTH OF COUNTY } \\
\text { TRUNK HIGHWAY A, } 2.9 \text { MI }(4.7 \mathrm{KM}) \\
\text { NORTHWEST OF RICHLAND CENTER. }\end{array}$ & 7.42 & $\begin{array}{l}06-03-74 \\
09-10-74 \\
05-14-75\end{array}$ & $\begin{array}{l}4.58 \\
3.43 \\
4.76\end{array}$ & 2.2 & 1.9 & $\mathrm{~b}$ \\
\hline 05406785 & RRUSH CREEK & $20 a$ & $\begin{array}{l}\text { NW } 1 / 4 \text { NW } 1 / 4 \text { SEC.13. T.10 N.. R.1 W.P } \\
\text { RICMLAND COUNTY, AT BRIDGE ON DEADEND } \\
\text { TOWNSHIP ROAO O.1 MI }(0.2 \text { KM) NORTH OF } \\
\text { U.S. HIGHWAY I4. } 3.1 \text { MI }(5.0 \mathrm{KM}) \text { WEST } \\
\text { OF RICHLAND CENTER. }\end{array}$ & $1 \cdot 29$ & $\begin{array}{l}06-03-74 \\
09-09-74 \\
05-14-75\end{array}$ & $\begin{array}{l}.85 \\
.42 \\
.84\end{array}$ & .15 & .10 & $b$ \\
\hline 05406786 & $\begin{array}{r}\text { RRUSH CREEK } \\
\text { TRIBUTARY }\end{array}$ & $20 \mathrm{~b}$ & $\begin{array}{l}\text { NE } 1 / 4 \text { NW } 1 / 4 \text { SEC.13, T.10 N.. R.1 H., } \\
\text { RICHLAND COUNTY AT RRIOGE ON U.S. } \\
\text { HIGHWAY } 1402.9 \text { MI } 14.7 \mathrm{KMI} \text { WEST OF } \\
\text { RICHLANO CENTER. }\end{array}$ & $1 \cdot 32$ & $\begin{array}{l}06-03-74 \\
09-09-74 \\
05-14-75\end{array}$ & $\begin{array}{r}1.08 \\
.40 \\
1.11\end{array}$ & .09 & .04 & $b$ \\
\hline 05406797 & BRUSH CREEK & $4 \mathrm{~b}$ & $\begin{array}{l}\text { NE } 1 / 4 \text { NE } 1 / 4 \text { SEC.13, T.10 N., R.I H.P } \\
\text { RICHLANO COUNTY, AT RRIDGE ON PRIVATE } \\
\text { ROAD. 2.5 MI }(4.0 \text { KM) WEST TF RICHLAND } \\
\text { CENTER. }\end{array}$ & 3.13 & $\begin{array}{l}06-03-74 \\
09-09-74 \\
05-14-75\end{array}$ & $\begin{array}{l}2.55 \\
1.94 \\
? .14\end{array}$ & 1.5 & 1.3 & b \\
\hline 05406795 & DINE RIVER & 23 & $\begin{array}{l}\text { SH } 1 / 4 \text { NH } 1 / 4 \text { SEC. } 21 \text { T.10 N.P R.1 E., } \\
\text { RICHLAND COUNTY, AT SEWAGE TREATMENT } \\
\text { PLANT, AT RICHLAND CENTER. }\end{array}$ & 190 & $\begin{array}{l}07-06-72 \\
10-18-72 \\
06-27-73 \\
08-22-73 \\
10-07-75\end{array}$ & $\begin{array}{l}74.3 \\
110 \\
189 \\
129 \\
87.6\end{array}$ & 65 & 55 & b \\
\hline $05406 A>0$ & DINE RIVER & 25 & $\begin{array}{l}\text { SF } 1 / 4 \text { SF } 1 / 4 \text { SEC.27. T.10 N.. R.1 E.. } \\
\text { RICHLAND COUNTY, AT RRIOGE ON COUNTY } \\
\text { TRINK HIGHWAY O. } 2.5 \text { MI (4. K KM) } \\
\text { SOUTHEAST OF RICHLAND CENTER. }\end{array}$ & $19 A$ & $07-30-65$ & ${ }^{2} 62.3$ & 74 & 63 & 10 \\
\hline 05406875 & $\triangle S H$ CREEK & $26 a$ & 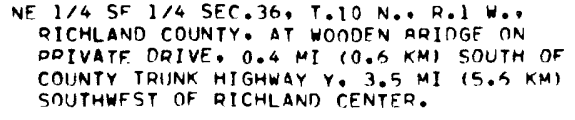 & 7.00 & $\begin{array}{l}06-03-74 \\
09-10-74 \\
05-14-75\end{array}$ & $\begin{array}{l}1.25 \\
1.36 \\
1.13\end{array}$ & a & a & \\
\hline 05406940 & $\triangle S H$ CRFEK & $26 b$ & 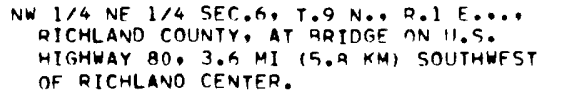 & $4 \cdot 174$ & $\begin{array}{l}06-03-74 \\
09-10-74 \\
05-14-75\end{array}$ & $\begin{array}{l}2.15 \\
2.18 \\
1.97\end{array}$ & a & a & \\
\hline OS 4 OnganO & ASH CREEK ${ }^{3}$ & 28 & $\begin{array}{l}\text { SE } 1 / 4 \text { NF } 1 / 4 \text { SEC.2. T.9 N.. R. I E... } \\
\text { RICHLAND COUNTY, AT RRTDGE ON COUNTY } \\
\text { TRINK HIGHWAY TR, } 4 \text {. M MI }(6.4 \text { KM) } \\
\text { SOITHEAST OF RICHLAND CENTER. }\end{array}$ & 12.4 & $09-07-57$ & 23.15 & 3.0 & 2.4 & 9 \\
\hline $0 S 404051$ & DINE RIVER & $28 \mathrm{a}$ & 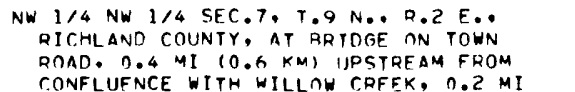 & 224 & $\begin{array}{l}06-04-74 \\
09-09-74 \\
05-14-75\end{array}$ & $\begin{array}{l}171 \\
133 \\
183\end{array}$ & 91 & 77 & $b$ \\
\hline
\end{tabular}


LOMER WISCONSIN RIVER BASIN

\begin{tabular}{|c|c|c|c|c|c|c|c|c|c|}
\hline \multirow[t]{2}{*}{$\begin{array}{l}\text { STATION } \\
\text { NO. }\end{array}$} & \multirow[t]{2}{*}{ STREAM NAME } & \multirow[t]{2}{*}{$\begin{array}{l}\text { PROJECT } \\
\text { NO. }\end{array}$} & \multirow{2}{*}{\multicolumn{2}{|c|}{ WILLOW CREEK AASIN }} & \multirow[t]{2}{*}{ DATE } & \multirow[t]{2}{*}{$\begin{array}{l}\text { DIS- } \\
\text { CHARGE } \\
\text { (FT /S) }\end{array}$} & \multicolumn{3}{|c|}{ 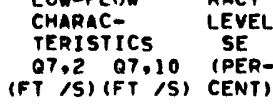 } \\
\hline & & & & & & & & & \\
\hline 05406853 & WILLOW CREEK & 1 & $\begin{array}{l}\text { SW } 1 / 4 \text { SE } 1 / 4 \text { SEC.10. T.11 N., R.2 E.. } \\
\text { RICHLAND COUNTY. O.3 MI }(0.5 \mathrm{KM}) \\
\text { UPSTREAM FROM BRIOGE ON STATE HIGHWAY } \\
58,1.2 \mathrm{MI}(1.9 \mathrm{KM}) \text { NORTH OF LOYD. }\end{array}$ & 8.91 & $\begin{array}{l}05-07-74 \\
08-09-76 \\
10-26-76\end{array}$ & $\begin{array}{l}4.59 \\
2.42 \\
2.30\end{array}$ & 1.8 & 1.4 & b \\
\hline 0540 RA55 & HAPPY HOLLOW CREEK & 2 & $\begin{array}{l}\text { NE } 1 / 4 \text { NW } 1 / 4 \text { SEC.15, T.11 N., R.2 E., } \\
\text { RICHLAND COUNTY, AT MOUTH. } 1.0 \mathrm{MI} \\
\text { I1.6 KMI NORTH OF LOYD. }\end{array}$ & 5.25 & $\begin{array}{l}05-07-74 \\
08-09-76 \\
10-26-76\end{array}$ & $\begin{array}{l}3.89 \\
2.03 \\
1.99\end{array}$ & 1.6 & 1.2 & b \\
\hline 05406858 & SMITH HOLLOW CREFK & 3 & $\begin{array}{l}\text { SH } 1 / 4 \text { SW } 1 / 4 \text { SEC.14, T.11 N.P R.2 E.. } \\
\text { RICMLAND COUNTY, } 200 \text { FT }(60 \mathrm{M}) \text { ABOVE } \\
\text { BRIDGE ON PRIVATE ROAD, } 1.4 \mathrm{MI}(2.2 \mathrm{KM}) \\
\text { EAST OF LOYD. }\end{array}$ & 3.72 & $\begin{array}{l}05-07-74 \\
08-09-76 \\
10-26-76\end{array}$ & $\begin{array}{r}.86 \\
.33 \\
.36\end{array}$ & .24 & .15 & b \\
\hline $054068 \times 0$ & $\begin{array}{l}\text { SMITH HOLLOW CRFEK } \\
\text { TRIBUTARY }\end{array}$ & 4 & $\begin{array}{l}\text { NE } 1 / 4 \text { NW } 1 / 4 \text { SEC.24. T.11 N., R.2 E., } \\
\text { RICHLAND COUNTY, O.6 MI (1.0 KM) } \\
\text { UPSTREAM FROM SMITH HOLLDW CREEK. } \\
2.0 \mathrm{MI}(3.2 \mathrm{KM}) \text { EAST OF LOYN. }\end{array}$ & $3 \cdot 42$ & $\begin{array}{l}05-07-74 \\
08-09-76 \\
10-26-76\end{array}$ & $\begin{array}{l}.75 \\
.21 \\
.25\end{array}$ & .14 & .09 & $\mathrm{~b}$ \\
\hline 05406867 & WHEAT HOLLOW CRFFK & 7 & $\begin{array}{l}\text { NW } 1 / 4 \text { NW } 1 / 4 \text { SEC. } 34 \text {. T.11 N.P R.2 E.P. } \\
\text { RICHLAND COUNTY, AT BRIDGE ON COUNTRY } \\
\text { ROAD, } 0.1 \text { MI }(0.2 \mathrm{KM}) \text { UPSTREAM FROM } \\
\text { MOUTH. } 2.1 \mathrm{MI}(3.4 \mathrm{KM}) \text { SOUTH OF LOYD. }\end{array}$ & 2.97 & $\begin{array}{l}05-07-74 \\
08-09-76 \\
10-26-76\end{array}$ & $\begin{array}{r}1.03 \\
.57 \\
.01\end{array}$ & .46 & .36 & $\mathrm{~b}$ \\
\hline 05406877 & LITTLE WILLOW CRFEK & 12 & $\begin{array}{l}\text { SW } 1 / 4 \text { NW } 1 / 4 \text { SEC.6, T.1O N., R.2 E.P. } \\
\text { RICHLANO COUNTY. AT RRIDGE ON COUNTRY } \\
\text { ROAB. } 2.0 \mathrm{MI}(3.2 \mathrm{KM}) \text { WEST NF NEPTIUNE. }\end{array}$ & 9.71 & $\begin{array}{l}05-07-74 \\
08-09-76 \\
10-26-76\end{array}$ & $\begin{array}{l}3.42 \\
1.97 \\
1.79\end{array}$ & 1.4 & 1.1 & $\mathrm{~b}$ \\
\hline 05406879 & $\begin{array}{l}\text { LITTLE WILLOW CREEK } \\
\text { TRIBUTARY }\end{array}$ & 13 & $\begin{array}{l}\text { SE } 1 / 4 \text { SW } 1 / 4 \text { SEC.7, T.IN N.. R.2 E... } \\
\text { QICHLAND COUNTY. AT MOIITH, } 1.4 \mathrm{MI} \\
\text { (2.2 KM) NORTHWEST OF ITHACA. }\end{array}$ & $? \cdot 32$ & $\begin{array}{l}05-07-74 \\
08-09-76 \\
10-26-76\end{array}$ & $\begin{array}{r}1.12 \\
.64 \\
.54\end{array}$ & .37 & .25 & $\mathrm{~b}$ \\
\hline 054 OKRR 4 & HELL HOLLOW CRFFK & 16 & 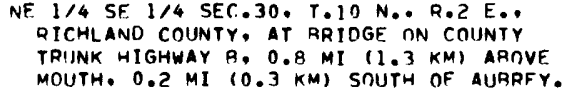 & $? .77$ & $05-07-74$ & 0.00 & 0.0 & 0.0 & $\mathrm{~b}$ \\
\hline 05404800 & WILLOW CPFFK' & $30 \mathrm{a}$ & 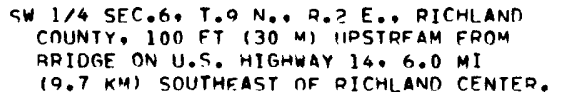 & A?. 5 & $07-30-45$ & ${ }^{2} 26.5$ & 31 & 27 & 8 \\
\hline & & & RLUE RIVEP BASIN & & & & & & \\
\hline$n n+0>n>0$ & $\begin{array}{l}\text { 2LIJE RIVFR } \\
\text { TFIRUTARY }\end{array}$ & & $\begin{array}{l}\text { NE } 1 / 4 \text { SW } 1 / 4 \text { SEC.24, TAR N., REI W.. } \\
\text { GRANT COUNTY, AT SEWAGE TREATMENT } \\
\text { PLANT. IN MONTFORT. }\end{array}$ & 0.58 & $\begin{array}{l}07-07-72 \\
06-28-73 \\
08-23-73 \\
10-08-75\end{array}$ & $\begin{array}{l}.55 \\
1.26 \\
1.1 ? \\
.64\end{array}$ & .36 & .26 & $\mathrm{~b}$ \\
\hline 06407011 & PLIIT HIVER & 4 & $\begin{array}{l}\text { NW } 1 / 4 \text { NW } 1 / 4 \text { SEC. } 2 \text {, T.6 N., R. } 1 \text { W. } \\
\text { GRANT COUNTY, AT BRIOGF ON TOWN ROAD. } \\
4.5 \mathrm{MI}(7.2 \mathrm{KM}) \text { WEST OF HIGHLANO. }\end{array}$ & 29.9 & $\begin{array}{l}10-19-72 \\
n 7-2 ?-76 \\
10-27-76\end{array}$ & $\begin{array}{l}12.5 \\
14.2 \\
12.7\end{array}$ & a & a & \\
\hline nqo.07n 21 & WIT SPPING PRANCH & 9 & $\begin{array}{l}\text { SE } 1 / 4 \text { NW } 1 / 4 \text { SEC.11. T.7 N.P R. I W., } \\
\text { GRANT COUNTY, AT BQINGF ON PRIVATE } \\
\text { ROAD, S.1 MI }(0.8 \mathrm{kM}) \text { SOUTH OF MUSCONA. }\end{array}$ & $1>0$ & $\begin{array}{l}10-19-72 \\
07-22-76 \\
10-27-76\end{array}$ & $\begin{array}{l}4.30 \\
5.13 \\
4.47\end{array}$ & a & a & \\
\hline $1+4.47021$ & WLIIF RTVFD & 10 & $\begin{array}{l}\text { SE } 1 / 4 \text { NF } 1 / 4 \text { SEC. 32, T.A N., R.1 W. } \\
\text { FRANT COUNTY AT RRIDGF ON COUNTY TRIJNK } \\
\text { HIGHWAY G, } 4.8 \text { MI }(7.7 \text { KMI SOUTHEAST OF } \\
\text { RLUE RIVER. }\end{array}$ & 79.6 & $\begin{array}{l}18-19-70 \\
10-17-72 \\
07-22-76 \\
10-27-76\end{array}$ & $\begin{array}{l}31.3 \\
35.5 \\
40.0 \\
35.9\end{array}$ & 27 & 21 & b \\
\hline 04,4070401 & FFNNIMURF FORK & 12 & 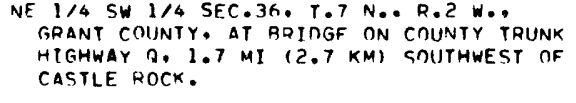 & $23 \cdot 3$ & $\begin{array}{l}10-18-72 \\
n 7-22-76 \\
10-27-76\end{array}$ & $\begin{array}{l}1.39 \\
.40 \\
.40\end{array}$ & .20 & .08 & b \\
\hline n) $F_{n}+0>n \rightarrow 0$ & FENNIMOPF FODK ${ }^{1}$ & & $\begin{array}{l}\text { SOUTH RDIINDARY SEC.31. T.A N.. R.I W. } \\
\text { GPANT COUNTY. AT BRINGF ON COUNTY TRUNK } \\
\text { HIGHWAY M, } 5.0 \text { MI (A.0 KM) SOUTH OF } \\
\text { RLUE RIVER. }\end{array}$ & 77.7 & $09-06-67$ & ${ }^{2} 19.3$ & 22 & 18 & $\mathrm{~b}$ \\
\hline$n \sim 407 n<1$ & SANE BPANCH & 19 & 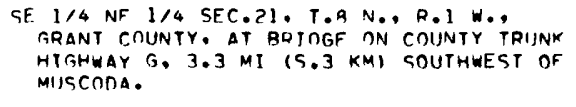 & 9.42 & $\begin{array}{l}08-19-70 \\
10-17-72 \\
07-23-76 \\
10-27-76\end{array}$ & $\begin{array}{l}1 \cdot 4 R \\
2 \cdot 86 \\
? \cdot 90 \\
2.72\end{array}$ & 1.8 & 1.3 & $\mathrm{~b}$ \\
\hline 0 म44070म? & $\begin{array}{l}\text { RLUE: HIVER } \\
\text { TRIMITADY }\end{array}$ & 20 & $\begin{array}{l}\text { SW } 1 / 4 \text { NF } 1 / 4 \text { SEC.15. T.9 N., R.I W. } \\
\text { GRANT COUNTY, AT RPINGF NN COUNTY TRUNK } \\
\text { HTGHAY G. 2.0 MI }(3.2 \mathrm{KM} \text { SOUTHWEST } \\
\text { OF MUSCODA. }\end{array}$ & $P .92$ & $10-17-72$ & 0.00 & 0 & 0 & $\mathrm{~b}$ \\
\hline $044070 \% 2$ & HLUE RIVER & 21 & 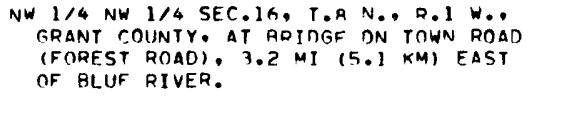 & $181 \cdot n$ & $\begin{array}{l}07-27-64 \\
07-30-55 \\
10-17-72 \\
07-73-76 \\
10-27-76\end{array}$ & $\begin{array}{l}56.4 \\
46.7 \\
77.1 \\
74.6 \\
72.1\end{array}$ & 58 & 47 & $\mathrm{~b}$ \\
\hline
\end{tabular}




\begin{tabular}{|c|c|c|c|c|c|c|c|c|c|}
\hline \multirow[t]{2}{*}{$\begin{array}{l}\text { STATION } \\
\text { NO. }\end{array}$} & \multirow[t]{2}{*}{ STREAM NAME } & \multirow[t]{2}{*}{$\begin{array}{c}\text { PROJECT } \\
\text { NO. }\end{array}$} & \multirow[b]{2}{*}{ PLATTE RIVER RASIN } & \multirow[t]{2}{*}{$\begin{array}{l}\text { DRAIN- } \\
\text { AGE } \\
\text { AREA } \\
\text { (MI2) }\end{array}$} & \multirow[t]{2}{*}{ DATE } & \multirow[t]{2}{*}{$\begin{array}{l}\text { DIS- } \\
\text { CHARGE } \\
\text { (FT3/S) }\end{array}$} & \multirow{2}{*}{\multicolumn{2}{|c|}{$\begin{array}{l}\text { LOW-FLOW } \\
\text { CHARAC- } \\
\text { TERISTICS } \\
\text { OT,2 } 97,10 \\
\left(F T^{3} / S\right)\left(F T^{3} / S\right)\end{array}$}} & \multirow[t]{2}{*}{$\begin{array}{l}\text { RACY } \\
\text { LEVEL } \\
\text { SE } 10 \\
\text { (PER } \\
\text { CENT) }\end{array}$} \\
\hline & & & & & & & & & \\
\hline 05413750 & PLATTE RIVER & 3 & $\begin{array}{l}\text { NE } 114 \text { SE } 1 / 4 \text { SEC.13, T.5 N., R.2 W.P } \\
\text { GRANT COUNTY, ON COUNTY TRUNK HIGHWAY } \\
\text { E, 4.1 MI }(6.6 \mathrm{KM}) \text { SOUTHEAST DF } \\
\text { STITZER. }\end{array}$ & 27.0 & $\begin{array}{l}09-18-69 \\
08-18-70 \\
09-01-71\end{array}$ & $\begin{array}{l}11.5 \\
11.4 \\
8.92\end{array}$ & a & a & \\
\hline 05413790 & CROW BRANCH & 5 & $\begin{array}{l}\text { NW } 1 / 4 \text { NW } 1 / 4 \text { SEC.19; T.5 N., R.1 W., } \\
\text { GRANT COUNTY, ON TOWN ROAD, } 4.4 \text { MI } \\
\text { (7.1 KM) SOUTHEAST OF STITZER. }\end{array}$ & 8.89 & $\begin{array}{l}09-18-69 \\
08-19-70 \\
09-01-71\end{array}$ & $\begin{array}{l}2.93 \\
1.94 \\
2.37\end{array}$ & 1.6 & 1.1 & $\mathrm{~b}$ \\
\hline 05413810 & LEGGETT CREEK & 9 & $\begin{array}{l}\text { SW } 1 / 4 \text { SW } 1 / 4 \text { SEC.13, T.5 N., R.? W., } \\
\text { GRANT COUNTY ON COUNTY TRUNK HIGHWAY } \\
\text { ER 3.5 MI }(5.6 \mathrm{KM}) \text { SOUTHEAST OF } \\
\text { STITZER. }\end{array}$ & 18.4 & $\begin{array}{l}09-18-69 \\
08-18-70 \\
09-01-71\end{array}$ & $\begin{array}{l}4.36 \\
5.34 \\
4.67\end{array}$ & $\mathbf{a}$ & $\mathbf{a}$ & \\
\hline 05413850 & $\begin{array}{l}\text { PLATTE RIVER } \\
\text { TRIBUTARY }\end{array}$ & 10 & $\begin{array}{l}\text { NW } 1 / 4 \text { SF } 1 / 4 \text { SEC. } 33, T^{T} .5 \text { N., R.2 W. } \\
\text { GRANT COUNTY, } 0.1 \text { MI }(n .2 \text { KM) } \\
\text { DOWNSTREAM FROM TOWN ROAD, } 5.1 \text { MI } \\
\text { (B.2 KM) EAST OF LANCASTER. }\end{array}$ & $13 \cdot 3$ & $\begin{array}{l}09-18-69 \\
08-18-70 \\
09-01-71\end{array}$ & $\begin{array}{l}2.61 \\
2.85 \\
3.38\end{array}$ & a & a & \\
\hline 05413890 & PACON BRANCH & 12 & $\begin{array}{l}\text { NE 1/4 SW } 1 / 4 \text { SEC.2, T.4 N., R.2 W.P. } \\
\text { GRANT COUNTY AT BRIDGE ON TOWN ROAD, } \\
4.7 \mathrm{MI}(7.6 \mathrm{KM}) \text { NORTHEAST OF ELLENBORO. }\end{array}$ & $7 \cdot 56$ & $\begin{array}{l}09-18-69 \\
08-17-70 \\
09-01-71\end{array}$ & $\begin{array}{r}1.49 \\
.75 \\
.71\end{array}$ & .35 & .16 & $\mathrm{~b}$ \\
\hline 05413900 & PLATTE RIVER & 13 & $\begin{array}{l}\text { NW } 1 / 4 \text { NW } 1 / 4 \text { SEC } 10 \text {, T.4 N., R.2 W. } \\
\text { GRANT COUNTY AT RRIDGE ON COUNTY TRUINK } \\
\text { HIGHWAY AP } 5.5 \text { MI }(8.9 \text { KM) EAST OF } \\
\text { LANCASTER. }\end{array}$ & $9 n \cdot A$ & $\begin{array}{l}09-18-69 \\
08-17-70 \\
09-01-71\end{array}$ & $\begin{array}{l}38.8 \\
32.6 \\
30.0\end{array}$ & 25 & 19 & $\mathrm{~b}$ \\
\hline 05413930 & AUSTIN BRANCH & 14 & $\begin{array}{l}\text { NW } 1 / 4 \text { SE 1/4 SEC.17, T.4 N., R.2 W., } \\
\text { SRANT COUNTY, AT MOUTH. } 2.7 \text { MI } 14.3 \mathrm{kM}) \\
\text { NORTHWFST OF ELLENAORO. }\end{array}$ & 5.52 & $06-21-72$ & ${ }^{2} 3.00$ & 1.6 & 1.0 & 22 \\
\hline 05413970 & MC PHERSON RRANCH & 16 & $\begin{array}{l}\text { SE } 1 / 4 \text { SE } 1 / 4 \text { SEC. } 29, T \text { T N., R.? W., } \\
\text { GRANT COUNTY AT BRIDGE ON TOWN ROAD, } \\
0.8 \mathrm{MI}(1.3 \mathrm{KM}) \text { WEST OF ELLENBORO. }\end{array}$ & 5.12 & $\begin{array}{l}09-18-69 \\
08-18-70 \\
09-31-71\end{array}$ & $\begin{array}{l}2.62 \\
2.56 \\
2.56\end{array}$ & $a$ & $a$ & \\
\hline 05414000 & PLATTE RIVER ${ }^{3}$ & & 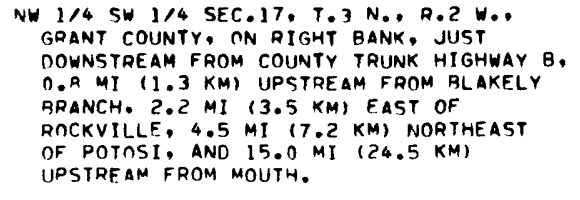 & $14 ?$ & $11-24-50$ & ${ }^{4} 0.00$ & 34 & 22 & 7 \\
\hline 09414010 & RLAKELY RRANCH & 19 & $\begin{array}{l}\text { NW } 1 / 4 \text { NE } 1 / 4 \text { SEC.2O. T. T N., R.? W.P } \\
\text { GRANT COUNTY, AT BRIDGE ON TOWN ROAD, } \\
\text { P.A MI }(4.2 \text { KM) EACT OF ROCKVILLE. }\end{array}$ & 2.68 & $\begin{array}{l}04-19-70 \\
08-31-71\end{array}$ & $\begin{array}{l}0.00 \\
0.00\end{array}$ & 0.0 & 0.0 & b \\
\hline 05414046 & IITTLE PLATTE DIVFD & & $\begin{array}{l}\text { SW } 1 / 4 \text { SE } 1 / 4 \text { SEC. } 24 \text {, T.5 N., R.I W.. } \\
\text { SRANT COUNTY, AT BRIDGE ON STATE } \\
\text { HIGHWAY SO. 1.0 MI (1.6 KM) SOIITH OF } \\
\text { LIVINGSTON. }\end{array}$ & 1.20 & $\begin{array}{l}06-23-72 \\
09-01-72 \\
08-03-73 \\
10-17-75 \\
07-26-76\end{array}$ & $\begin{array}{r}.25 \\
1.21 \\
1.13 \\
.40 \\
.17\end{array}$ & .09 & .03 & $\mathrm{~b}$ \\
\hline 05414150 & LITTLE PLATTF RIVFD' & 8 & $\begin{array}{l}\text { SW } 1 / 4 \text { SE } 1 / 4 \text { SEC.4, T.3 N. PR.1 W., } \\
\text { GRANT CNUNTY ON STATE HIGHWAY } 81 \text {, } \\
1.9 \text { MI IJ.1 KM) NORTHWEST OF } \\
\text { PI.ATTEVILLE. }\end{array}$ & $54 \cdot 4$ & $09-07-57$ & 26.70 & 5.6 & 2.8 & 21 \\
\hline $0541479 n$ & DEAR BRANCH? & 9 & $\begin{array}{l}\text { NW } 1 / 4 \text { SFC. } 4 \text { T T } 3 \text { N., D. } W \text { W GRANT } \\
\text { COINTY, AT BOX CULVFRT ON STATF } \\
\text { HIGHAY } 81,2.3 \text { MI }(3.7 \text { KMI NORTHWFST } \\
\text { OF PLATTEVILLE. }\end{array}$ & 2.90 & $09-07-67$ & ${ }^{2} \cdot 49$ & .62 & .36 & 14 \\
\hline $04414 ? 25$ & YOINNG ARANCH & 10 & $\begin{array}{l}\text { NW } 1 / 4 \text { SE } 1 / 4 \text { SEC.K, T.3 N. D R.1 W. } \\
\text { GRANT COUNTY AT RRIDGF ON TOWN ROAD, } \\
3.3 \text { MI } 15.3 \text { KMI NORTHWFST OF } \\
\text { PLATTEVILLE. }\end{array}$ & $3 \cdot 34$ & $\begin{array}{l}09-17-69 \\
09-19-70 \\
09-02-71\end{array}$ & $\begin{array}{l}.35 \\
.10 \\
.20\end{array}$ & .12 & .07 & $\mathrm{~b}$ \\
\hline 05414210 & ROUNOTREE BRANCH & & $\begin{array}{l}\text { SE } 1 / 4 \text { NF } 1 / 4 \text { SEC.17, T.3 N.. R.1 W. } \\
\text { GRANT COUNTY AT SEWAGE TREATMENT PLANT } \\
\text { IN PLATTEVILLE. }\end{array}$ & $1>\cdot 5$ & $\begin{array}{l}08-30-72 \\
06-28-73 \\
08-02-73 \\
10-16-75 \\
07-26-76\end{array}$ & $\begin{array}{r}4.75 \\
1 ? .4 \\
8.94 \\
4.94 \\
6.83\end{array}$ & 1.7 & .90 & $b$ \\
\hline $054142>0$ & IITTLE PLATTE RTVFD & 15 & $\begin{array}{l}\text { NE } 1 / 4 \text { SF } 1 / 4 \text { SEC.II. T.? N.P R.Z W.P. } \\
\text { GRANT COISNTY AT RRIDGF ON TOWN ROAD. } \\
2.5 \text { MI }(4.0 \text { KMI NORTHEAST OF } \\
\text { DICKFYVILLE. }\end{array}$ & 96.6 & $\begin{array}{l}0 Q-18-69 \\
n P-18-70 \\
n R-30-71\end{array}$ & $\begin{array}{l}40.1 \\
25.9 \\
20.8\end{array}$ & 16 & 10 & $\mathrm{~b}$ \\
\hline 04414250 & ALOCKHOUSF CRFFK' & & $\begin{array}{l}\text { SW } 1 / 4 \text { SFC.11. T.2 N.OB R.2 W. . GRANT } \\
\text { COIJNTY, AT BRIDGE ON U.S. } 4 \text { IGHWAY } 151 \text {, } \\
2.5 \mathrm{MI}(4.0 \mathrm{KM}) \text { NORTH IF DICKEYVILLE. }\end{array}$ & $36 \cdot 3$ & $09-07-66$ & ${ }^{2} 4.10$ & 6.9 & 3.9 & 17 \\
\hline 05414278 & INDIAN CREEK & & $\begin{array}{l}\text { SW } 1 / 4 \text { NF } 1 / 4 \text { SEC.29, T.P N., R.? W.. } \\
\text { GRANT COUNTY, AT BRIDGF ON INWN ROAD, } \\
\text { P.D MI }(3.4 \text { KM) WFST OF DICKEYVILLE. }\end{array}$ & 3.72 & $\begin{array}{l}n 6-22-72 \\
0 B-30-72\end{array}$ & $\begin{array}{l}2.05 \\
5.01\end{array}$ & $<.01$ & $<.01$ & $\mathrm{~b}$ \\
\hline
\end{tabular}




\begin{tabular}{|c|c|c|c|c|c|c|c|c|c|}
\hline \multirow[t]{2}{*}{$\begin{array}{l}\text { STATION } \\
\text { NO. }\end{array}$} & \multirow[t]{2}{*}{ STREAM NAME } & \multirow[t]{2}{*}{$\begin{array}{l}\text { PROJECT } \\
\text { NO. }\end{array}$} & \multirow[b]{2}{*}{ WHITEWATER CREEK BASIN } & \multirow[t]{2}{*}{$\begin{array}{l}\text { DRAIN- } \\
\text { AGE } \\
\text { AAEA } \\
\text { (MI 2) }\end{array}$} & \multirow[t]{2}{*}{ DATE } & \multirow[t]{2}{*}{$\begin{array}{l}\text { DTS- } \\
\text { CHARGE } \\
\text { (FT3/S) }\end{array}$} & \multirow{2}{*}{\multicolumn{2}{|c|}{$\begin{array}{l}\text { LOW-FLOW } \\
\text { CHARAC- } \\
\text { TERISTICS } \\
07,2 \text { Q } 97,10 \\
\text { (FT3/S) }(F T 3 / 5)\end{array}$}} & \multirow[t]{2}{*}{$\begin{array}{l}\text { RACY } \\
\text { LEVEL } \\
\text { SE } \\
\text { IPEQ } \\
\text { CENTI }\end{array}$} \\
\hline & & & & & & & & & \\
\hline 05426500 & WHITEWATER CAEEK ${ }^{3}$ & 2 & $\begin{array}{l}\text { SW } 104 \text { NW 1/4 SEC.26, T.4 N., R.15 E., } \\
\text { WALWORTH COUNTY, AT OUTLET OF RICE } \\
\text { LAKE, ON TOWN ROAD, } 4.1 \text { MI }(6.6 \mathrm{KM}) \\
\text { SOUTH OF WHITEWATER. }\end{array}$ & -- & $\begin{array}{l}07-18-54 \\
70 \\
07-28-54\end{array}$ & 0.00 & 0.43 & 0.17 & 32 \\
\hline 05426900 & WHI TEWATER CAEEK & 5 & $\begin{array}{l}\text { NE } 1 / 4 \text { SE } 1 / 4 \text { SEC, } 15, T_{14} N_{1}{ }^{R} 15 \text { E.. } \\
\text { WALWORTH COUNTY, } 2.5 \text { MI }(4,0 \text { KM) } \\
\text { SOUTHEAST OF POST OFFICE AT WHITEWATER. }\end{array}$ & 20.5 & $\begin{array}{l}09-24-71 \\
16-27-72 \\
09-17-75\end{array}$ & $\begin{array}{l}11.6 \\
11.7 \\
15.9\end{array}$ & 9.0 & 6.8 & b \\
\hline 05427000 & WHITEWATER CRFEK ${ }^{3}$ & 6 & $\begin{array}{l}\text { NE } 1 / 4 \text { SW } 1 / 4 \text { SEC.10, T.4 N., R.15 E., } \\
\text { WALWORTH COUNTY, ON TOWN ROAD, } 1.5 \mathrm{MI} \\
(2.4 \mathrm{KM}) \text { SOUTHFAST OF WHITEWATER. }\end{array}$ & $27 \cdot 7$ & $01-17-50$ & 46.60 & 10 & 7.6 & 13 \\
\hline $054270 n 2$ & SPRING BROOK & 8 & $\begin{array}{l}\text { SE } 1 / 4 \text { NE } 1 / 4 \text { SEC. } 22 \text { T.4 N. R. R.14 E. } \\
\text { ROCK COUNTY. AT BRIDGE AT JUNCTION OF } \\
\text { STATE HIGHWAY S9 AND COUNTRY ROAD, } \\
\text { O.5 MI } 10.8 \mathrm{KMI} \text { NORTHEAST OF LIMA } \\
\text { CENTER. }\end{array}$ & $\cdot 18$ & $\begin{array}{l}09-25-71 \\
09-18-75\end{array}$ & $\begin{array}{l}0.00 \\
0.00\end{array}$ & 0.00 & 0.00 & $b$ \\
\hline 05427009 & $\begin{array}{l}\text { SPRING BROOK } \\
\text { TRIBUTARY }\end{array}$ & 13 & $\begin{array}{l}\text { NE 1/4 SF 1/4 SEC.18, T.4 N.P R.15 E.. } \\
\text { WALWORTH COUNTY, AT CULVERT ON TOWN } \\
\text { ROAD, } 2.5 \text { MI }(4.0 \text { KM) SOUTHWEST OF } \\
\text { WHITEWATER. }\end{array}$ & 2.06 & $\begin{array}{l}09-24-71 \\
09-19-75\end{array}$ & $\begin{array}{l}0.00 \\
0.00\end{array}$ & 0.00 & 0.00 & $b$ \\
\hline 05427010 & SPRING BROOK & 14 & $\begin{array}{l}\text { NW 1/4 SE } 1 / 4 \text { SEC.8, T.4 N.. R.15 E.. } \\
\text { WALWRTH COUNTY ON STATE HIGHWAY B9, } \\
1.2 \text { MI } 11.9 \text { KM) SOUTHWEST OF POST } \\
\text { OFFICE AT WHITEWATER. }\end{array}$ & 16.7 & $\begin{array}{l}09-24-71 \\
06-26-72 \\
09-18-75\end{array}$ & $\begin{array}{l}2.38 \\
4.95 \\
4.96\end{array}$ & 1.5 & .80 & $b$ \\
\hline 05427013 & WHITEWATER CREFK & 15 & $\begin{array}{l}\text { NW } 1 / 4 \text { NW } 1 / 4 \text { SEC. } 4 \text {, T.4 N., R.15 E.P̈, } \\
\text { WALWORTH COUNTY, AT RRIDGE ON PRIVATF } \\
\text { ROAD, AT COUNTY LINE, O.6 MI (1.0 KMI } \\
\text { NORTH OF POST OFFICE AT WHITEWATER. }\end{array}$ & $47 \cdot 6$ & $09-25-71$ & ${ }^{2} 14.3$ & 12 & 9.2 & 16 \\
\hline 05427014 & $\begin{array}{l}\text { WHITENATER CDFFK } \\
\text { TRIBUTARY }\end{array}$ & 16 & $\begin{array}{l}\text { NW 1/4 NW } 1 / 4 \text { SEC.34, T.5 N., R.15 E. } \\
\text { JEFFERSON COIJNTY ON STATE HIGHWAY SO. } \\
\text { 1.7 MI }(2.7 \text { KM N NORTHEAST OF POST } \\
\text { OFFICE AT WHITEWATER. }\end{array}$ & 7.05 & $\begin{array}{l}09-25-71 \\
09-17-75 \\
10-17-73\end{array}$ & $\begin{array}{r}.24 \\
.36 \\
2.14\end{array}$ & .09 & .04 & b \\
\hline 05427015 & $\begin{array}{l}\text { TRIBUTARY TO } \\
\text { WHITFWATER CAFFK } \\
\text { TRTHUTARY }\end{array}$ & 17 & $\begin{array}{l}\text { SF. } 1 / 4 \text { SW } 1 / 4 \text { SEC.28, T.5 N.P R.15 E.. } \\
\text { JEFFERSON COUNTY, ON COUNTY TRUNK } \\
\text { HITHWAY U, I.7 MI I } 2.7 \text { KM) NORTH OF } \\
\text { POST OFFICE AT WHITFHATER. }\end{array}$ & - 25 & $\begin{array}{l}09-25-71 \\
09-17-75\end{array}$ & $\begin{array}{l}0.00 \\
0.00\end{array}$ & 0.00 & 0.00 & $\mathrm{~b}$ \\
\hline 05427017 & WHITEWATFR CRFFK & 18 & $\begin{array}{l}\text { SE. } 1 / 4 \text { SF } 1 / 4 \text { SEC.29, T. } 5 \text { N., R.15 E.P } \\
\text { JFFFERSON COUNTY, AT BRIDGE NN COUNTY } \\
\text { TRUNK HIGHWY U. } 1.7 \text { MI }(2.7 \text { KM) NORTH } \\
\text { OF POST OFFICE AT WHTTFHATER. }\end{array}$ & 55.4 & $\begin{array}{l}n 9-14-56 \\
10-17-73 \\
n 9-16-75\end{array}$ & $\begin{array}{l}15.4 \\
43.6 \\
24.7\end{array}$ & 13 & 9.8 & $\mathrm{~b}$ \\
\hline 04427019 & GALLCWAY CREFK & 19 & $\begin{array}{l}\text { NF 1/4 SF } 1 / 4 \text { SEC.35, T.S N.. R.14 E.. } \\
\text { JFFFERSON COIINTY AT CULVERT ON TOWN } \\
\text { ROADE } 3.5 \text { MI }(5.6 \text { KM) WEST OF } \\
\text { WHITEWATER. }\end{array}$ & 5.95 & $\begin{array}{l}n 9-24-71 \\
n 9-18-75\end{array}$ & $\begin{array}{r}0.00 \\
.08\end{array}$ & 0.00 & 0.00 & b \\
\hline $054270>11$ & $\begin{array}{l}\text { r.ALLSWAY CAFFK } \\
\text { TQIPUTARY }\end{array}$ & 20 & 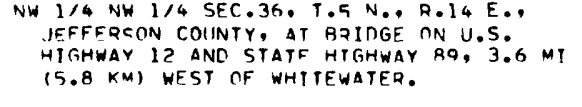 & .90 & $\begin{array}{l}09-24-71 \\
09-18-75\end{array}$ & $\begin{array}{l}0.00 \\
0.00\end{array}$ & 0.00 & 0.00 & b \\
\hline 04427027 & $\begin{array}{l}\text { FALLCWAY CPEFE } \\
\text { TPIBUTARY }\end{array}$ & 24 & $\begin{array}{l}\text { SE } 1 / 4 \text { SW } 1 / 4 \text { SEC.24, T.5 N., R.14 E. } \\
\text { JFFFFRSON COIJNTY. AT PRINGE ON TOWN } \\
\text { ROAD. } 4 \text { O MI (K.4 KM) NORTHWEST OF } \\
\text { WHITFWATER. }\end{array}$ & - GA & $\begin{array}{l}09-24-71 \\
09-18-75\end{array}$ & $\begin{array}{l}0.00 \\
0.00\end{array}$ & 0.00 & 0.00 & $\mathrm{~b}$ \\
\hline $1044>1036$ & $\begin{array}{l}\text { FALLTWAY CRFFK } \\
\text { TRIRIITARY }\end{array}$ & 27 & $\begin{array}{l}\text { SE 1/4 NW 1/4 SEC.6, T.4 N., R.15 E., } \\
\text { WALWNRTH COUNTY, AT RRIOGE ON U.Sं } \\
\text { HIGHWAY 1? AND STATF HIGHWAY R9, 1.8 MI } \\
\text { (2.9 KM) WEST OF POST OFFICE AT } \\
\text { WHITEWATFR. }\end{array}$ & -- & $\begin{array}{l}n 9-24-71 \\
09-19-75\end{array}$ & $\begin{array}{r}0.00 \\
.04\end{array}$ & 0.00 & 0.00 & $\mathrm{~b}$ \\
\hline 09427035 & $\begin{array}{l}\text { FALLC.WAY CREFK } \\
\text { TZIBIITARY }\end{array}$ & 29 & 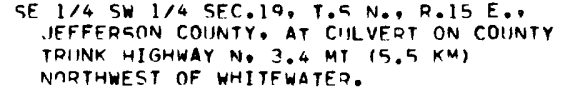 & -- & $\begin{array}{l}09-25-71 \\
09-16-75\end{array}$ & 0.01 & 0.00 & 0.00 & b \\
\hline 05427077 & WHITEWATFP CDFFK & 30 & $\begin{array}{l}\text { NF } 1 / 4 \text { SW } 1 / 4 \text { SEC.1B, T.5 N., R.15 F., } \\
\text { IFFFFRCON COUNTY, AT BRIOGE ON COUNTY } \\
\text { TRIINK HIGHWAY DN, AT COLD SPRING. }\end{array}$ & 6R. & $\begin{array}{l}08-15-67 \\
11-17-73 \\
09-16-75\end{array}$ & $\begin{array}{l}16.8 \\
54 \cdot 9 \\
25.2\end{array}$ & 17 & 13 & $\mathrm{~b}$ \\
\hline
\end{tabular}


LOWER ROCK RIVER BASIN

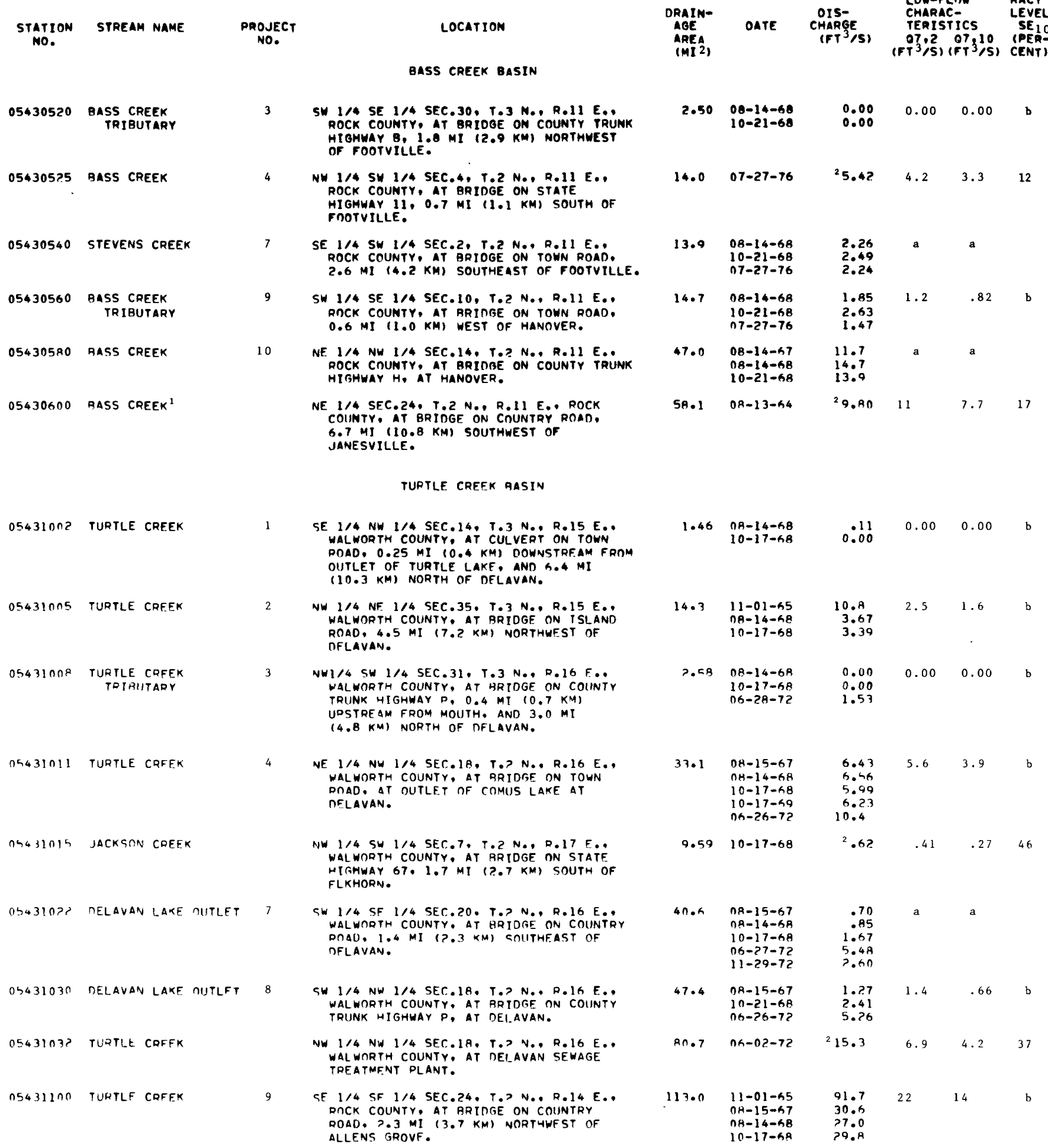


TABLE 12.--BASIN PROJECT SITE LOCATIONS AND LOH-FLOW CHARACTERISTICS

LOWER ROCK RIVER BASIN

\begin{tabular}{|c|c|c|c|c|c|c|c|c|c|c|c|}
\hline $\begin{array}{l}\text { STATION } \\
\text { NO. }\end{array}$ & STREAM & a NAME & & $\begin{array}{l}\text { PAOJECT } \\
\text { NO. }\end{array}$ & LOCATION & $\begin{array}{l}\text { DRAIN- } \\
\text { AGE } \\
\text { ADEA } \\
\text { (MI } 2 \text { ) }\end{array}$ & DATE & $\begin{array}{l}\text { OIS- } \\
\text { CHARGE } \\
\left(F^{3} / S\right)\end{array}$ & $\begin{array}{l}\text { LOW-F } \\
\text { CHARA } \\
\text { TERIS } \\
07 \cdot 2 \\
\left(F T^{3} / S\right)\end{array}$ & $\begin{array}{l}\text { LOW } \\
\text { C- } \\
\text { TiCs } \\
\text { Q7:10 } \\
\left(F T^{3} / S\right)\end{array}$ & $\begin{array}{l}\text { ACCU- } \\
\text { RACY } \\
\text { LEVEL } \\
\text { SE } \\
\text { IPER } \\
\text { CENT) }\end{array}$ \\
\hline 05431125 & LITTLE & TURTLE & CREEK & & $\begin{array}{l}\text { NW } 1 / 4 \text { SE } 1 / 4 \text { SEC.32, T.1 N., R.15 E., } \\
\text { WALWORTH COUNTY, AT SEWAGE TREATMENT } \\
\text { PLANT, } 1.0 \mathrm{MI} 11.6 \mathrm{KMI} \text { WEST OF SHARON. }\end{array}$ & 1.71 & $\begin{array}{l}06-02-72 \\
07-25-73 \\
11-06-73 \\
09-22-75\end{array}$ & $\begin{array}{l}.05 \\
.19 \\
.44 \\
.02\end{array}$ & $<.01$ & $<.01$ & b \\
\hline 05431250 & LADD CR & REEK & & 12 & $\begin{array}{l}\text { SW 1/4 SF 1/4 SEC.17, T.1 N., R.15 E. } \\
\text { WALWORTH COUNTY, AT BRIOFE' ON SALT BOX } \\
\text { ROAD, 3.0 MI }(4.8 \mathrm{KM}) \text { SOUTH OF } \\
\text { ALLENS GROVE. }\end{array}$ & 6.73 & $\begin{array}{l}n 8-14-68 \\
10-17-68 \\
0 K-26-72\end{array}$ & $\begin{array}{r}.75 \\
.63 \\
1.26\end{array}$ & .40 & .20 & b \\
\hline 05431400 & LITTLE & TURTLE & CREEK & & $\begin{array}{l}\text { NE } 1 / 4 \text { SEC.L. T.1 N., R.15 E., WALWORTH } \\
\text { COUNTY, AT BRINGE ON COUNTRY ROAO, } \\
0.2 \mathrm{MI}(0.3 \mathrm{kM}) \text { SOUTH OF ALLENS GROVE. }\end{array}$ & $41 \cdot A$ & $07-30-65$ & ${ }^{2} \cdot 30$ & 1.6 & .54 & 47 \\
\hline $054314>0$ & $\begin{array}{l}\text { LITTLE } \\
\text { TRIBU }\end{array}$ & $\begin{array}{l}\text { TURTLE } \\
\text { JTARY }\end{array}$ & CREFK & 16 & $\begin{array}{l}\text { NE 1/4 SF 1/4 SEC.32, T.2 N., R.15 E.. } \\
\text { WALWORTH COUNTY, AT CULVERT ON STATE } \\
\text { HIGHWAY 15, } 1.2 \text { MI } 11.0 \text { KMI EAST OF } \\
\text { ALLENS GROVE. }\end{array}$ & 11.0 & $\begin{array}{l}08-14-68 \\
10-17-68 \\
06-26-72\end{array}$ & $\begin{array}{l}1.84 \\
1.69 \\
2.16\end{array}$ & a & a & \\
\hline 05431500 & TURTLE & CREEK $^{3}$ & & & $\begin{array}{l}\text { SW } 1 / 4 \text { SE } 1 / 4 \text { SEC.29. T.? N.. R.14 E., } \\
\text { ROCK COUNTY, AT STATE HIGHWAY } 140, \\
2.5 \text { MI }(4.0 \mathrm{KM}) \text { NORTH OF CLINTON. }\end{array}$ & $20 ?$ & $1 \geq-29-56$ & ${ }^{4} 8.00$ & 38 & 26 & 7 \\
\hline 05431550 & $\begin{array}{l}\text { TURTLE } \\
\text { TRIRU }\end{array}$ & $\begin{array}{l}\text { CREEK } \\
\text { JTARY }\end{array}$ & & 20 & $\begin{array}{l}\text { SE } 1 / 4 \text { SW } 1 / 4 \text { SEC. } 35, T .2 N \text { N., R.13 E., } \\
\text { ROCK COUNTY, AT BRIOGE ON COUNTRY ROAD, } \\
\text { R.3 MI }(0.5 \text { KM) UPSTREAM FROM MOUTH, } \\
\text { ANR } 1.1 \text { MI }(1.8 \text { KM) NORTHFAST OF } \\
\text { SHOPIERE. }\end{array}$ & $7 \cdot 28$ & $\begin{array}{l}08-14-58 \\
10-17-68\end{array}$ & $\begin{array}{l}0.00 \\
0.00\end{array}$ & 0.00 & 0.00 & b \\
\hline 05431650 & TURTLE & CDF.EK & & 22 & $\begin{array}{l}\text { NE } 1 / 4 \text { NH } 1 / 4 \text { SEC. } 9 \text {, T.1 N., R.13 E., } \\
\text { ROCK COUNTY, AT BRIDGE ON LATHERS ROAD. } \\
1.3 \text { MI }(2.1 \text { KM) WEST OF SHOPIERE. }\end{array}$ & $2>3$ & $\begin{array}{l}08-15-67 \\
08-14-68 \\
10-17-68\end{array}$ & $\begin{array}{l}41.0 \\
49.3 \\
43.4\end{array}$ & a & a & \\
\hline 05431700 & TURTLE & CREEK & & 23 & $\begin{array}{l}\text { SH 1/4 SW } 1 / 4 \text { SEC.30, T.1 N.. R.13 E., } \\
\text { ROCK COUNTY, AT BRIDGE ON STATE } \\
\text { HIGHWAY 15, AT BELOIT. }\end{array}$ & 237 & $\begin{array}{l}08-14-67 \\
08-14-68 \\
10-17-68\end{array}$ & $\begin{array}{l}34.1 \\
49.2 \\
45.0\end{array}$ & a & a & \\
\hline
\end{tabular}

${ }^{1}$ LOW-FLOW PARTIAL-RECORD STATION.

${ }^{2} M I N I M U M$ MEASURED

${ }^{3}$ GAGING STATION.

${ }^{4}$ MINIMUM RECORDED.
${ }^{8}$ RESULT OF BRIDGE CONSTRUCTION. 
PECATONICA RIVER BASIN

\begin{tabular}{|c|c|c|c|c|c|c|c|c|c|}
\hline \multirow[t]{2}{*}{$\begin{array}{l}\text { STATION } \\
\text { NO. }\end{array}$} & \multirow[t]{2}{*}{ STREAM NAME } & \multirow[t]{2}{*}{$\begin{array}{l}\text { PROJECT } \\
\text { NO. }\end{array}$} & \multirow[b]{2}{*}{ JONES BRANCH BASIN } & \multirow[t]{2}{*}{$\begin{array}{l}\text { ORAIN- } \\
\text { ARE } \\
\text { AREA } \\
\text { (MI I ) }\end{array}$} & \multirow[t]{2}{*}{ DATE } & \multirow[t]{2}{*}{$\begin{array}{l}\text { DIS- } \\
\text { CHARGE } \\
\text { (FT } 3 / S)\end{array}$} & \multirow{2}{*}{\multicolumn{2}{|c|}{$\begin{array}{l}\text { CHARAC- } \\
\text { TERISTICS } \\
\text { Q7,2 } Q 7,10 \\
\text { (FT3/S) }\left(F T^{3} / S\right)\end{array}$}} & \multirow[t]{2}{*}{$\begin{array}{l}\text { RACY } \\
\text { LEVEL } \\
\text { SE } 10 \\
\text { (PER- } \\
\text { CENT) }\end{array}$} \\
\hline & & & & & & & & & \\
\hline 05432080 & $\begin{array}{l}\text { JONES BRANCH } \\
\text { TRIBUTARY }\end{array}$ & 3 & $\begin{array}{l}\text { SW 1/4 NW 1/4 SEC.26, T.4 N., R.1 E.: } \\
\text { LAYFAYETTE COUNTY, } 1.8 \mathrm{MI}(2.9 \mathrm{KM}) \\
\text { NORTHEAST OF LESLIE. }\end{array}$ & .99 & $\begin{array}{l}08-17-70 \\
08-31-71\end{array}$ & $\begin{array}{l}0.00 \\
0.00\end{array}$ & 0.00 & 0.00 & b \\
\hline 05432088 & $\begin{array}{l}\text { JONES BRANCH } \\
\text { TRIBUTARY }\end{array}$ & 7 & $\begin{array}{l}\text { SE 1/4 SE 1/4 SEC.23, T.4 N., R.1 E... } \\
\text { LAFAYETTE COUNTY 0.1 MI }(0.2 \mathrm{KM}) \\
\text { UPSTREAM FROM MOUITH, } 2.7 \mathrm{MI}(4.3 \mathrm{KM}) \\
\text { NORTHEAST OF LESLIE. }\end{array}$ & 0.44 & $\begin{array}{l}08-17-70 \\
n 8-31-71\end{array}$ & $\begin{array}{l}0.00 \\
0.00\end{array}$ & 0.00 & 0.00 & $\mathbf{b}$ \\
\hline \multirow[t]{2}{*}{$0543 ? 099$} & $\begin{array}{l}\text { JONES BRANCH } \\
\text { TRIBUTARY }\end{array}$ & 8 & $\begin{array}{l}\text { GE. 1/4 NF 1/4 SEC.23, T.4 N., R.1 E... } \\
\text { LAFAYETTE COUNTY, AT BRIDGE ON YOWN } \\
\text { ROAD, 7.1 MI } 15.0 \text { KM) NORTHEAST OF } \\
\text { LESLIE. }\end{array}$ & 0.16 & $\begin{array}{l}08-17-70 \\
08-31-71\end{array}$ & $\begin{array}{l}0.00 \\
0.00\end{array}$ & 0.00 & 0.00 & b \\
\hline & & & OTTER CREEK RASIN & & & & & & \\
\hline $054325 \cap 4$ & $\begin{array}{l}\text { OTTER CREEK } \\
\text { TRIBUTARY }\end{array}$ & 3 & $\begin{array}{l}\text { SE } 1 / 4 \text { SE } 1 / 4 \text { SEC.10, T.4 N., R.3 E.P. } \\
\text { IOWA COUNTY, AT ARIDGE ON TOWN ROAD, } \\
3.8 \text { MI }(6.1 \text { KMI SOUTHEAST OF MINERAL } \\
\text { POINT. }\end{array}$ & 1.51 & $\begin{array}{l}08-17-70 \\
09-01-71\end{array}$ & $\begin{array}{l}0.00 \\
0.00\end{array}$ & 0.00 & 0.00 & b \\
\hline $054325 n 5$ & $\begin{array}{l}\text { OTTER CREEK } \\
\text { TRIBUTARY }\end{array}$ & $3 a$ & $\begin{array}{l}\text { SW } 1 / 4 \text { NF } 1 / 4 \text { SEC.15, T.4 N.. R. } 3 \text { E., } \\
\text { IOWA COUNTY. AT BRIDGE ON COUNTY TRUNK } \\
\text { HIGHWAY } 5,3.9 \text { MI }(6.3 \mathrm{KM}) \text { WFST OF } \\
\text { WALDWICK. }\end{array}$ & 1.79 & $\begin{array}{l}08-17-70 \\
09-01-71\end{array}$ & $\begin{array}{l}0.00 \\
0.00\end{array}$ & 0.00 & 0.00 & b \\
\hline 05432506 & $\begin{array}{l}\text { DTTER CREFK } \\
\text { TRIBUTARY }\end{array}$ & 4 & 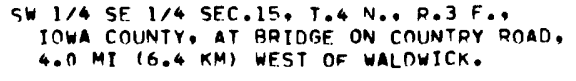 & 1.98 & $\begin{array}{l}08-17-70 \\
09-01-71\end{array}$ & $\begin{array}{l}0.00 \\
0.00\end{array}$ & 0.00 & 0.00 & $\mathrm{~b}$ \\
\hline 05432507 & $\begin{array}{l}\text { TRIBUTARY TO } \\
\text { OTTER CREEK } \\
\text { TRIHIITARY }\end{array}$ & 5 & $\begin{array}{l}\text { NE } 1 / 4 \text { NW } 1 / 4 \text { SEC.22, T.4 N., R. } 3 \text { F.. } \\
\text { LAYFAYFTTE COUNTY, AT RRIDGF ON TOWN } \\
\text { ROAD, } 4.1 \text { MI } 16.6 \mathrm{KM}) \text { WEST OF WALOWICK. }\end{array}$ & $1 .>5$ & $\begin{array}{l}08-17-70 \\
09-01-71\end{array}$ & $\begin{array}{l}0.00 \\
0.00\end{array}$ & 0.00 & 0.00 & $\mathrm{~b}$ \\
\hline $0343 ? 5 \cap 4$ & OTTER CREFK & 6 & $\begin{array}{l}\text { NW } 1 / 4 \text { NW } 1 / 4 \text { SEC.26. T.4 N.P R.3 F. } \\
\text { LAYFAYETTE. COUNTY, AT RRIDGE ON TOWN } \\
\text { ROADQ } 3.9 \text { MI } 16.3 \text { KM) SOUTHWFST OF } \\
\text { WALDWICK. }\end{array}$ & $R \cdot 36$ & $\begin{array}{l}n 8-18-70 \\
n 9-01-71 \\
n a-13-76\end{array}$ & $\begin{array}{l}2.19 \\
? .97 \\
1.42\end{array}$ & 2.3 & 1.3 & b \\
\hline 05432500 & $\begin{array}{r}\text { OTTER CPEFK } \\
\text { TRIRIJTADY }\end{array}$ & 7 & $\begin{array}{l}\text { SW } 1 / 4 \text { SF } 1 / 4 \text { SEC.1. T.4 N.P P.3 E. } \\
\text { INWA COUNTY AY BRIDGE ON TOWN POAD, } \\
\text { ?.? MI }(3.5 \mathrm{KMI} \text { NORTHWFST OF WALOWICK. }\end{array}$ & .97 & $\begin{array}{l}n A-17-70 \\
09-01-71\end{array}$ & $\begin{array}{l}0.00 \\
0.00\end{array}$ & 0.00 & 0.00 & $\mathrm{~b}$ \\
\hline 05437511 & $\begin{array}{l}\text { OTTEP CQEFK } \\
\text { TRI BUTADY }\end{array}$ & 8 & $\begin{array}{l}\text { SE } 1 / 4 \text { SW } 1 / 4 \text { SEC.?3. T.4 N., P. } 3 \text { F. } \\
\text { LAYFAYETTE COUNTY. AT ARIDGF ON TOWN } \\
\text { ROAD } 3.7 \text { MI (6.0 KM) SOUTHWEST OF } \\
\text { WALDWICK. }\end{array}$ & 3.65 & $\begin{array}{l}08-18-70 \\
n 0-01-71 \\
09-13-76\end{array}$ & $\begin{array}{r}.96 \\
1.05 \\
.07\end{array}$ & .92 & .68 & $\mathrm{~b}$ \\
\hline 09432415 & $\begin{array}{l}\text { OTTEL CREFK } \\
\text { THTLUTAARY }\end{array}$ & 11 & $\begin{array}{l}\text { SF } 1 / 4 \text { SF } 1 / 4 \text { SEC. } 35 . \text { T.4 N.. P. } 3 \text { F.. } \\
\text { LAYFAYFTE COUNTY. AT MOUTH. 3.? MI } \\
15.1 \text { KMI WEST OF FAYETTF. }\end{array}$ & 1.40 & $\begin{array}{l}n A-18-70 \\
n 9-01-71 \\
n 9-13-76\end{array}$ & $\begin{array}{l}.15 \\
.>7 \\
.12\end{array}$ & .16 & .08 & $\mathrm{~b}$ \\
\hline 05417414 & $\begin{array}{l}\text { OTTER CPFFK } \\
\text { THIMIITARY }\end{array}$ & 15 & $\begin{array}{l}\text { SW 1/4 NW } 1 / 4 \text { SEC. } 17, T .7 \mathrm{~N} . \text {. R.4 E. } \\
\text { LAYFAYETTF COUNTY, AT PRIDGE ON COINTY } \\
\text { TRINK HIGHWAYF, I.6 MT }(2.6 \mathrm{KM}) \\
\text { SOUTHWFST OF FAYETTE. }\end{array}$ & $1 \cdot 71$ & $\begin{array}{l}n 4-18-70 \\
0 R-31-71\end{array}$ & $\begin{array}{l}0.00 \\
0.00\end{array}$ & 0.00 & 0.00 & b \\
\hline$n+4+3>5>0$ & $\begin{array}{l}\text { TTEFF CPFFK } \\
\text { TAISIITAQY }\end{array}$ & 16 & 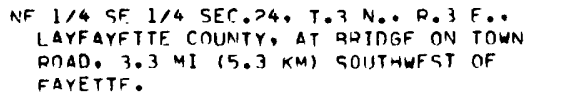 & 5.25 & $\begin{array}{l}n R-1 B-70 \\
n B-31-71 \\
n 9-13-76\end{array}$ & $\begin{array}{l}.19 \\
.31 \\
.04\end{array}$ & .14 & .02 & b \\
\hline $054375>?$ & $\begin{array}{l}\text { OTTEF CPFFK } \\
\text { TRI YIITAQY }\end{array}$ & 18 & 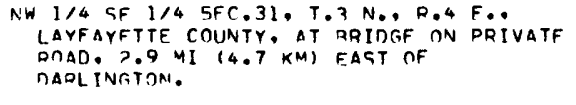 & 9.? 1 & $\begin{array}{l}29-18-70 \\
29-31-71 \\
n 9-13-76\end{array}$ & $\begin{array}{r}1.17 \\
1.34 \\
.69\end{array}$ & 1.0 & .38 & b \\
\hline $054325>3$ & $\begin{array}{l}\text { TDIBLTAPY TO } \\
\text { OTTEN CDFEK } \\
\text { THIHIITARY }\end{array}$ & 19 & 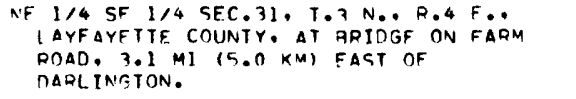 & 1.21 & $\begin{array}{l}n 8-18-70 \\
n x-31-71\end{array}$ & $\begin{array}{l}0.00 \\
0.00\end{array}$ & 0.00 & 0.00 & $\mathrm{~b}$ \\
\hline
\end{tabular}




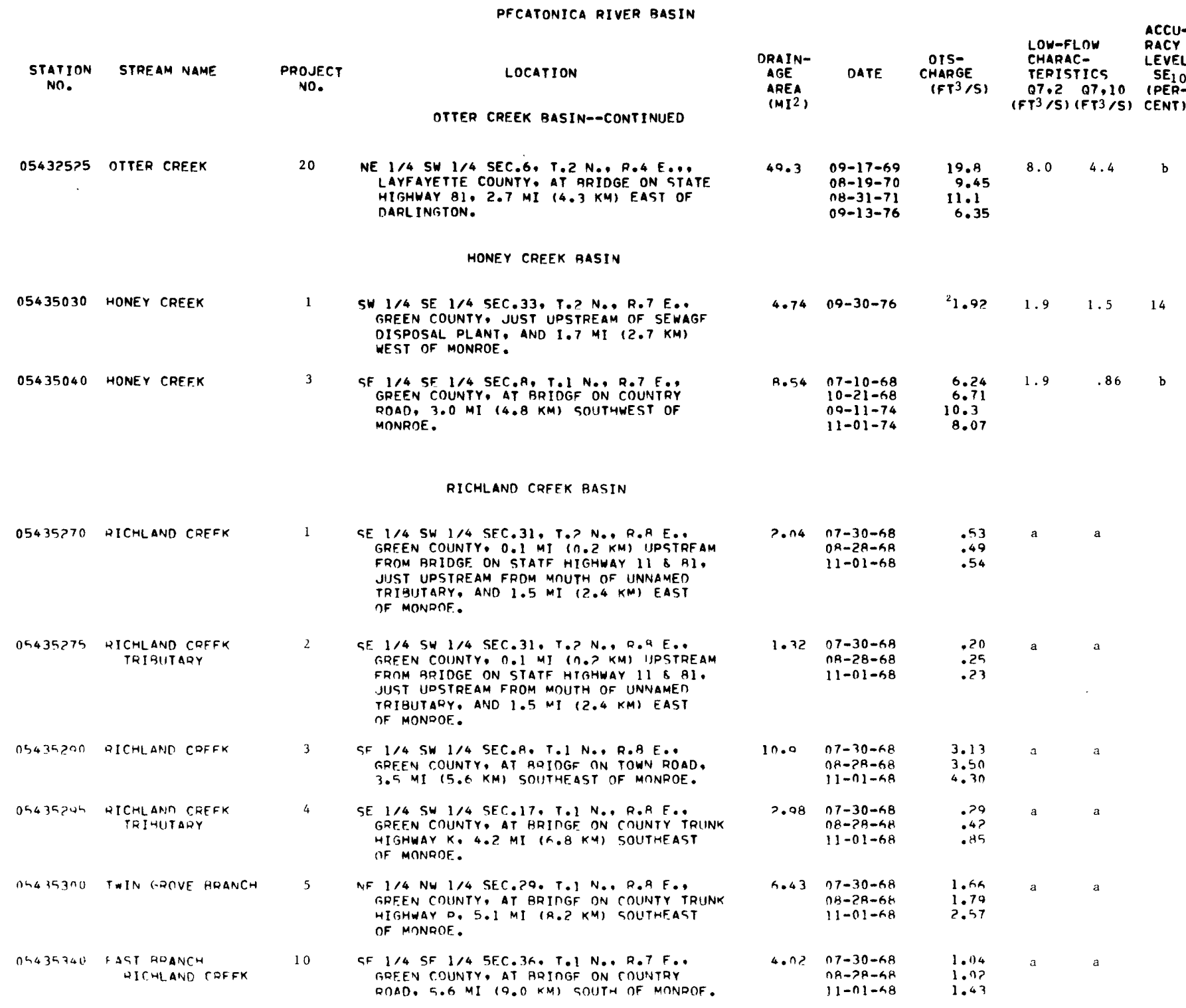

2MTNTMUTI MEASURED. 


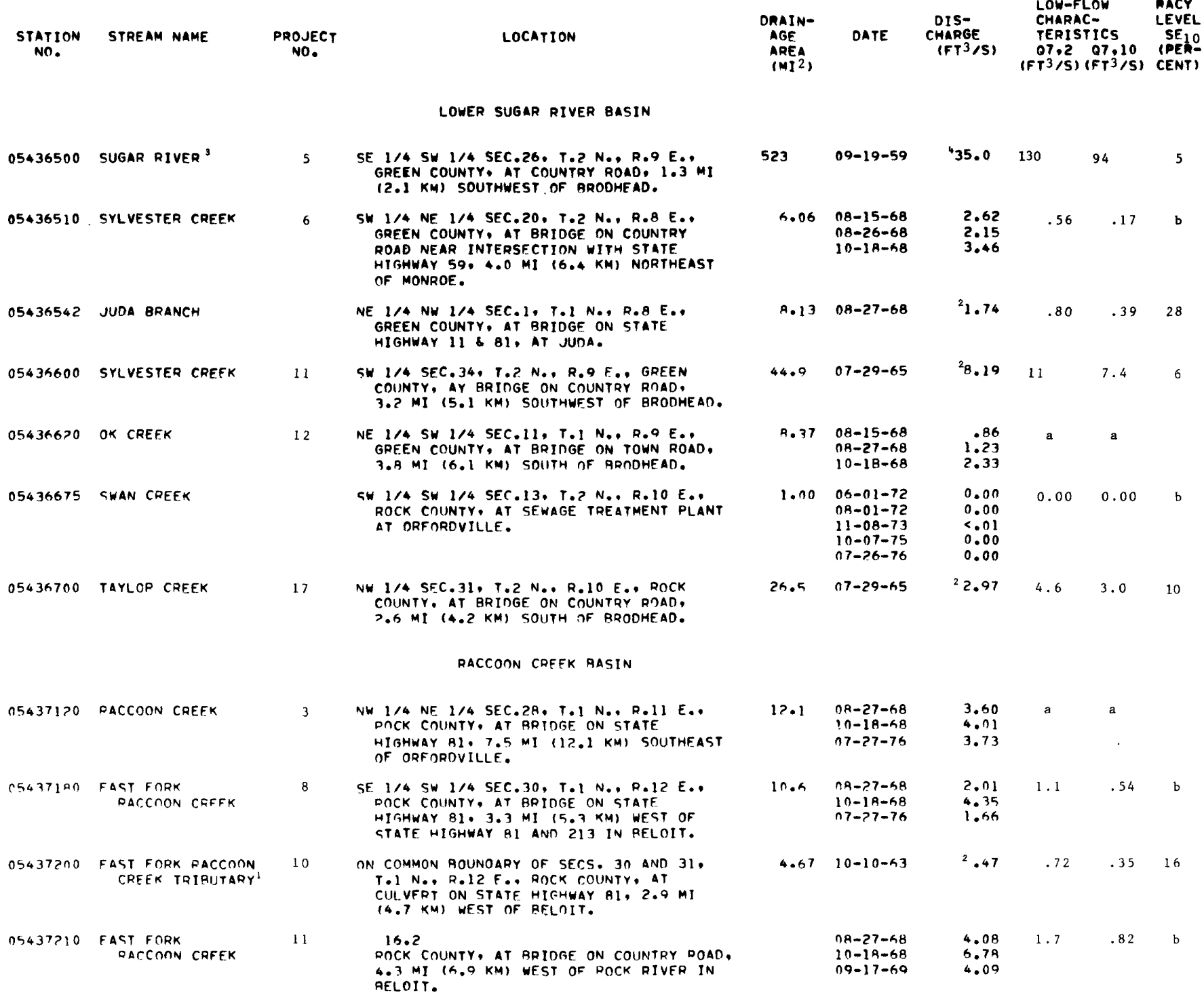


FOX RIVER (ILLINOIS) BASIN

\begin{tabular}{|c|c|c|c|}
\hline \multirow[t]{2}{*}{$\begin{array}{l}\text { STATION } \\
\text { NO. }\end{array}$} & \multirow[t]{2}{*}{ STREAM NAME } & \multirow[t]{2}{*}{$\begin{array}{l}\text { PROJECT } \\
\text { NO. }\end{array}$} & \multirow[b]{2}{*}{ HONEY-SUGAR CREEK BASIN } \\
\hline & & & \\
\hline 05544838 & HONEY CREEK & 5 & $\begin{array}{l}\text { SE } 1 / 4 \text { NE } 1 / 4 \text { SEC.25, T.4 N.9 R.17 E.. } \\
\text { WALWORTH COUNTY, AT CULVERT ON TOWN } \\
\text { ROAD, } 1.1 \text { MI }(1.8 \mathrm{KM}) \text { WEST OF EAST } \\
\text { TROY. }\end{array}$ \\
\hline 05544860 & $\begin{array}{l}\text { HONEY CREEK } \\
\text { TRIBUTARY }\end{array}$ & 7 & $\begin{array}{l}\text { NE } 1 / 4 \text { NF } 1 / 4 \text { SEC.15, T.4 N., R.18 E., } \\
\text { WAL WORTH COUNTY, AT STATE HIGHWAY } 24 \text {, } \\
3.6 \mathrm{MI}(5.8 \mathrm{KM}) \text { SOUTH DF MUKWONAGO. }\end{array}$ \\
\hline 05544910 & $\begin{array}{l}\text { HONEY CREEK } \\
\text { TRIBUTARY }\end{array}$ & 10 & $\begin{array}{l}\text { SE } 1 / 4 \text { SW } 1 / 4 \text { SEC. } 11 \text { T.4 N. P R.18 E. } \\
\text { WALWORTH COUNTY, ON STATE HIGHWAY } 24 \text {, } \\
3.4 \mathrm{MI}(5.5 \mathrm{KM}) \text { SOUTH OF MUKWONAGO. }\end{array}$ \\
\hline 05544970 & HONEY CREEK & 12 & $\begin{array}{l}\text { SE } 1 / 4 \text { NE } 1 / 4 \text { SEC. I, T. } 3 \text { N., R.1A E.., } \\
\text { WALWORTH COUNTY, AT BRIOGE ON COUNTY } \\
\text { TRUNK HIGHWAY D. AT HONEY CREEK. }\end{array}$ \\
\hline 05544990 & HONEY CREEK & 13 & $\begin{array}{l}\text { AF. } 1 / 4 \text { SE } 1 / 4 \text { SEC.13, T.3 N.A R.17 E., } \\
\text { WALWORTH COUNTY, MT BRIDGE ON COUNTY } \\
\text { TRUNK HIGHWAY DO. 3.1 MI }(5.0 \mathrm{KM}) \\
\text { NORTHWEST OF GURLINGTON. }\end{array}$ \\
\hline 05545080 & SUGAR CREEK & 14 & 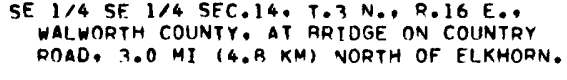 \\
\hline 05545109 & SUGAR CREEK & 17 & $\begin{array}{l}\text { SW } 1 / 4 \text { NE } 1 / 4 \text { SEC.15, T.3 N.. R.17 E., } \\
\text { WRLWORTH COUNTY, AT BRIOGE ON COUNTRY } \\
\text { ROAD. } 3.9 \text { MI (6.3 KM) NORTHWEST OF } \\
\text { SPRING PRAIRIE. }\end{array}$ \\
\hline 05545110 & SUGAR CRFEK & 18 & $\begin{array}{l}\text { NW } 1 / 4 \text { SW } 1 / 4 \text { SEC.8, T.3 N., R.1A E.P } \\
\text { WALWNRTH COUNTY, AT BRTDGE ON COUNTY } \\
\text { TRUNK HIGHWAY G, } 3.9 \mathrm{MI}(6.3 \mathrm{KM}) \text { EAST } \\
\text { OF TROY. }\end{array}$ \\
\hline 05545118 & $\begin{array}{l}\text { SUGAR CREFK } \\
\text { TRIBUTARY }\end{array}$ & 19 & $\begin{array}{l}\text { NW } 1 / 4 \text { SW } 1 / 4 \text { SEC. } 15.1 \text { T. } 3 \text { N.PR.18 E.. } \\
\text { WALWORTH COUNTY ON TOWN DOAO, } \\
3.0 \text { MT }(4.8 \text { KM) WEST OF HONEY LAKE. }\end{array}$ \\
\hline $055451>0$ & SUGAR CRFEK' & 20 & $\begin{array}{l}\text { SW } 1 / 4 \text { SW } 1 / 4 \text { SEC. } 14, \text { T. } 3 \text { N., R.1B E., } \\
\text { WALWORTH COUNTY, AT RRIDGE ON TOWN } \\
\text { ROAD, } 1.4 \text { MI (?.3 KM) WEST OF VIENNA. }\end{array}$ \\
\hline
\end{tabular}

\begin{tabular}{|c|c|c|c|c|}
\hline $\begin{array}{l}\text { ORAIN- } \\
\text { AGE } \\
\text { AREA } \\
\text { (MI } 2 \text { ) }\end{array}$ & DATE & $\begin{array}{l}\text { DIS- } \\
\text { CHARGE } \\
\left(F^{3} / S\right)\end{array}$ & $\begin{array}{l}\text { LOW-FLOW } \\
\text { CHARAC- } \\
\text { TERISTICS } \\
\text { Q7, } 2 \text { Q7, } 10 \\
\left(F T^{3} / 5\right)\left(F^{3} / 5\right)\end{array}$ & $\begin{array}{l}\text { ACCU- } \\
\text { RACY } \\
\text { LEVEL } \\
\text { SEIO } \\
\text { (PER- } \\
\text { CENT) }\end{array}$ \\
\hline
\end{tabular}

$\begin{array}{llllll}41.0 & 11-03-65 & 21.7 & 6.7 & 4.6 & b \\ 09-17-74 & 24.5 & & & \\ 09-17-75 & 19.7 & & & \\ & & & & & \\ 0.92 & 06-27-72 & 0.00 & 0.00 & 0.00 & b\end{array}$

$1.46 \quad 06-27-72 \quad 0.02 \quad 0.00 \quad 0.00$

74.? $09-13-66 \quad 10.2 \quad 9.2 \quad 5.8$ $08-15-6$ $06-27-72$

10.2
12.7
18.9

12.7
18.9

84.7 11-12-63 $11-12-63$
$06-27-72$ 23.4 $09-18-75$

14.0
25.4
29.6

27.? $\quad 09-13-56$ n6-26-72

1.95
4.05
4.83

$55.5 \quad 11-03-65$

09-13-66

$n R-15-67$

$n a-15-67$
$06-27-72$ $09-16-75$

18.1

4.89

6.24

11.7

61.5 11-03-65

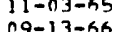

21.6 n9-16-75

14.3 $4.93 \quad n 9-13-66$
$n x-26-72$ $09-17-75$

1.55
$? .77$

71.? 07-30-65

26.22

$9.9 \quad 6.2 \quad b$

$1.7 \quad 1.1$

$4.8 \quad 3.1 \quad$ b

$9.8 \quad 6.5 \quad 17$

${ }^{1}$ LOW-FLOW PARTIAL-RECORD STATION.

MINIMUM MEASURED. 
Table 16.--Standard error of estimate for the 10-year low flow $\left(Q_{1}, 10\right)$
for major Wisconsin basins at low-flow partial-record stations (Gebert and Holmstrom, 1974, p. 98)

\begin{tabular}{|c|c|}
\hline Basin & $\mathrm{SE}_{7,10}$ in percent \\
\hline Bad Axe River & 10 \\
\hline Baraboo River & 11 \\
\hline Black River & 38 \\
\hline Lower Chippewa River & 25 \\
\hline Upper Chippewa River & 40 \\
\hline Duck-Pensaukee Rivers & 52 \\
\hline Fox River (Illinois) & 38 \\
\hline Lower Fox River & 44 \\
\hline Upper Fox River & 20 \\
\hline Grant, Platte, and Galena Rivers & 21 \\
\hline La Crosse River & 5 \\
\hline Lemonweir River & 15 \\
\hline Manitowoc River & 48 \\
\hline Menominee River & 28 \\
\hline Milwaukee River & 40 \\
\hline Oconto River & 17 \\
\hline Pecatonica River & 16 \\
\hline Peshtigo River & 17 \\
\hline Lower Rock River & 36 \\
\hline Upper Rock River & 71 \\
\hline Root River & $14 \overline{4}$ \\
\hline Sheboygan River & 48 \\
\hline St. Croix River & 28 \\
\hline Sugar River & 12 \\
\hline Lake Superior & 35 \\
\hline Trempealeau-Buffalo Rivers & 13 \\
\hline Twin-Kewaunee Rivers and & \\
\hline Door County & Insufficient data \\
\hline Lower Wisconsin River & 11 \\
\hline Upper Wisconsin River & 33 \\
\hline Wolf River & 23 \\
\hline Average (weighted) & 29 \\
\hline
\end{tabular}




\section{REFERENCES}

Gebert, W. A., 1971, Low-flow frequency of Wisconsin streams: U.S. Geological Survey Hydrologic Investigations Atlas HA-390.

Gebert, W. A., and Holmstrom, B. K., 1974, Low-flow characteristics of Wisconsin streams at sewage-treatment plants: U.S. Geological Survey Water-Resources Investigations 45-74, 101 p., 2 figs., 32 tables.

Hardison, C. H., 1969, Accuracy of streamflow characteristics, in Geological Survey Research 1969: U.S. Geological Survey Professional Paper 650D, p. D210-214.

Hardison, C. H., and Moss, M. E., 1972, Accuracy of low-flow characteristics estimated by correlation of base-flow measurements: U.S. Geological Survey Water-Supply Paper 1542-B, 55 p.

Riggs, H. C., 1972, Low-flow investigations: U.S. Geological Survey Techniques of Water-Resources Investigations, book 4, chapter BI, $18 \mathrm{p}$.

1968, Some statistical tools in hydrology: U.S. Geological Survey Techniques of Water-Resources Investigations, book 4, chapter Al, $39 \mathrm{p}$.

Soil and Water Conservation Districts, 1973, Guidelines for interagency cooperation with county soil and water conservation districts in Wisconsin watershed projects: Madison, $54 \mathrm{p}$. 
Subbasin

Bass Creek basin

Bear Creek basin

Beaver Creek basin

Blue River basin

De Nevue Creek basin

Duck Creek basin

East Branch Fond du Lac

River basin

East River basin

Embarrass River basin

Honey Creek basin

Honey-Sugar Creek basin

Jones Branch basin

Kewaunee River basin

Lower Fox River basin

Lower Sugar River basin

Mecan River basin

Montreal River basin

Neenah Slough basin

Otter Creek basin

Pigeon Creek basin

Pike River basin

Pine River basin

Platte River basin

Raccoon Creek basin

Richland Creek basin

Shioc River basin

Suamico River basin

Turtle Creek basin

West Branch Fond du Lac

River basin

Whitewater Creek basin

Willow Creek basin

Wolf River basin

\section{INDEX}

Major basin

$\underline{\text { Page }}$

Lower Rock River basin 24

Wolf River basin 14

Black River basin 18

Lower Wisconsin River basin 21

Upper Fox River basin 12

Duck-Pensaukee River basin 9

Upper Fox River basin 11

Lower Fox River basin 15

Wolf River hasin 14

Pecatonica River basin 27

Fox River (Illinois) basin 29

Pecatonica River basin 26

Twin-Kewaunee River basin 16

and Door County

Lower Fox River basin 15

Sugar River basin 28

Upper Fox River basin 10

Lake Superior basin 8

Upper Fox River basin 12

Pecatonica River basin 26

Trempealeau-Buffalo River basin 18

Des Plaines and Pike River basin 17

Lower Wisconsin River basin 20

Grant-Platte River basin 22

Sugar River basin 28

Pecatonica River basin 27

Wolf River basin 13

Duck-Pensaukee River basin 9

Lower Rock River basin 24

Upper Fox River basin 10

Lower Rock River basin 23

Lower Wisconsin River basin 21

Wolf River basin 13 